University of Louisville

ThinkIR: The University of Louisville's Institutional Repository

Electronic Theses and Dissertations

8-2013

\title{
A stage-structured delayed reaction-diffusion model for competition between two species.
}

Chunwei Wang

University of Louisville

Follow this and additional works at: https://ir.library.louisville.edu/etd

\section{Recommended Citation}

Wang, Chunwei, "A stage-structured delayed reaction-diffusion model for competition between two species." (2013). Electronic Theses and Dissertations. Paper 1521.

https://doi.org/10.18297/etd/1521

This Doctoral Dissertation is brought to you for free and open access by ThinkIR: The University of Louisville's Institutional Repository. It has been accepted for inclusion in Electronic Theses and Dissertations by an authorized administrator of ThinkIR: The University of Louisville's Institutional Repository. This title appears here courtesy of the author, who has retained all other copyrights. For more information, please contact thinkir@louisville.edu. 


\title{
A STAGE-STRUCTURED DELAYED REACTION-DIFFUSION MODEL FOR COMPETITION BETWEEN TWO SPECIES
}

\author{
By \\ Chunwei Wang \\ B.S., Beijing University of Technology, 2005 \\ M.A., University of Louisville, 2011

\begin{abstract}
A Dissertation
Submitted to the Faculty of the

College of Arts and Sciences of the University of Louisville

in Partial Fulfillment of the Requirements

for the Degree of
\end{abstract}

Doctor of Philosophy

Department of Mathematics

University of Louisville

Louisville, KY

August 2013 
Copyright 2013 by Chunwei Wang

All rights reserved 



\title{
A STAGE-STRUCTURED DELAYED REACTION-DIFFUSION MODEL FOR COMPETITION BETWEEN TWO SPECIES
}

\author{
Submitted by \\ Chunwei Wang
}

A Dissertation Approved on

June 5, 2013

(Date)

by the Following Reading and Examination Committee:

Bingtuan Li, Dissertation Director

\begin{tabular}{c}
\hline Elizabeth Bradley \\
\hline Ryan Gill \\
\hline Changbing $\mathrm{Hu}$ \\
\hline
\end{tabular}

Susanna Remold 


\section{ACKNOWLEDGMENTS}

I would like to thank my dissertation advisor, Dr. Bingtuan Li, for his guidance, patience, encouragement and leadership. It has been a precious experience to collaborate with him and have him as a mentor. I thank my committee members for their support and encouragement throughout.

I wish to thank my family for providing me with unconditional love and unsurpassed support no matter where we are. In particular, I thank my mom, Wenjie Wang, for her always being so supportive on not only my study but also

my life and dream. I thank my dad, Yiming Wang, for showing me the way to pursue my goal with my passion and diligence. I thank my husband, Roger Ouch, for his patience, love, support and always believing in me. 


\section{ABSTRACT \\ A STAGE-STRUCTURED DELAYED REACTION-DIFFUSION MODEL FOR COMPETITION BETWEEN TWO SPECIES \\ Chunwei Wang}

June 5, 2013

We formulate a delayed reaction-diffusion model that describes competition between two species in a stream. We divide each species into two compartments, individuals inhabiting on the benthos and individuals drifting in the stream. Time delays are incorporated to measure the time lengths from birth to maturity of the benthic populations. We assume that the growth of population takes place on the benthos and that dispersal occurs in the stream. Our system consists of two linear reaction-diffusion equations and two delayed ordinary differential equations. We study the dynamics of the non-spatial model, determine the existence and global stability of the equilibria, and provide conditions under which solutions converge to the equilibria. We show that the existence of traveling wave solutions can be established through compact integral operators. We define two real numbers and prove that they serve as the lower bounds of the speeds of traveling wave solutions in the system. 


\section{TABLE OF CONTENTS}

\section{CHAPTER}

1. INTRODUCTION AND BACKGROUND . . . . . . . . . . . . 1

1.1 Introduction . . . . . . . . . . . . . . . . . 1

1.2 Reaction-Diffusion Equations . . . . . . . . . . . . 5

1.3 Delay Differential Equations . . . . . . . . . . . . . . . 7

2. THE MODEL ......................... 16

2.1 Delayed Reaction-Diffusion Equations

For Single Species With Stage Structure . . . . . . . . . . 16

2.2 Two Species Lotka-Volterra-type Competition Model . . . . . . 18

3. NONSPATIAL SYSTEM ANALYSIS . . . . . . . . . . . . . . 22

3.1 Nonspatial System . . . . . . . . . . . . . . . 22

3.2 Local Stability . . . . . . . . . . . . . . . . . 23

3.3 Global Stability . . . . . . . . . . . . . . . 23

3.3.1 Global Stability of the mono-culture equilibria $E_{1}$ and $E_{2} \ldots \ldots \ldots \ldots$. . . . . . . . 27

3.3.2 Global Stability of the coexistence state $E^{*}$. . . . . . 29

4. EXISTENCE OF TRAVELING WAVES . . . . . . . . . . . . 35

4.1 Integral System . . . . . . . . . . . . . . . . . . 35

4.2 Traveling Wave Solutions . . . . . . . . . . . . . . . . . . 39

4.2.1 Recursions With Delay . . . . . . . . . . . . . . 39

4.2.2 Existence of Traveling Wave Solutions . . . . . . . . . 43

5. CONCLUSION AND FUTURE DIRECTIONS . . . . . . . . . . . 61 
5.1 Conclusion .................... 61

5.2 Discussion . . . . . . . . . . . . . . . . 62

5.3 Future Directions . . . . . . . . . . . . . . 63

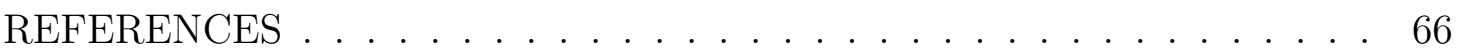

CURRICULUM VITAE . . . . . . . . . . . . . . . . . 75 


\section{LIST OF FIGURES}

1.1 Traveling wave solutions of FKPP equation, $\frac{\partial u}{\partial t}=D \frac{\partial^{2} u}{\partial x^{2}}+R(u)$, where $R=u-u^{2} \ldots \ldots \ldots \ldots$. . . . . . . . . 7

1.2 Solutions of the Hutchinson equation, $\frac{d x(t)}{d t}=r x(1-x(t-$ $\tau) / K)$, where $\tau=1,3 \ldots \ldots \ldots$. . . . . . . . 8

1.3 Spread of the solution of FKPP equation with the speed of $c^{*}=2 \sqrt{D R^{\prime}(0)} \ldots \ldots \ldots \ldots \ldots \ldots$

1.4 Traveling wave solution of the reaction-diffusion equation $u(x, t)=$ $w(x-c t)$ with $w(-\infty)=1$ and $w(+\infty)=0$ exists if and only if $c \geq c^{*} \ldots \ldots \ldots \ldots \ldots \ldots$ 


\section{CHAPTER 1 INTRODUCTION AND BACKGROUND}

\section{$1.1 \quad$ Introduction}

Many species, populations, communities, and ecosystems persist in environments where they spend some or all their life stages dispersing in media with strong directional bias. Typical examples include plants with windborn seeds, aquatic organisms in streams, and marine organisms with larval dispersal influenced by ocean currents. We focus on aquatic organisms living in streams, rivers, and estuaries which are characterized by unidirectional net water movement. Species living in such environments are facing the so-called "drift paradox" [1]. "Drift paradox" is one key issue for theory in stream ecology and states that extinction is inevitable in a closed population subjected only to downstream drift. We derive the ecological niche for persistence and spread of populations to avoid being swept downstream and out into habitats, where the physical or biotic environment is not conducive to their growth and reproduction.

Various hypotheses involving some compensatory upstream movement have been proposed as resolutions of the drift paradox. Müller [2, 3] claimed that adult insects balance out the downward drift of the insect larvae by flying upstream for oviposition. Waters [4] assumed that the drift paradox would be resolved if the species resided mainly on the benthos (the bottom of the stream) and only the surplus over the carrying capacity would drift in the stream. Persistence in 
streams could also be affected by some movement mechanisms which include refugia in streams $[5,6,7,8,9,10]$ and variability effect in stream flow direction such as turbulence [38]. Speirs and Gurney [11, 12] stated that insect swimming in the water column and crawling on the benthos may permit the persistence of the population. They also pointed out that Water's hypothesis [4] neatly sidesteps the problem of how lotic species are able to sustain population losses due to drift, and implicit in their view is that drifting is merely a source of mortality. Experimental studies have been conducted to provide evidence both supporting $[1,4,14]$ and contradicting $[4,15,16]$ to the first hypothesis of upstream flight recolonization. However, adult flight patterns strongly depend on the species that we considered, and this hypothesis does not cover many important species often found in the drift that have no aerial phases. For the statement of insects swimming in the stream and crawling on the benthos, some experimental data were obtained $[4,17,18,19]$, but those experiments were not conducted for the purpose of solving the drift paradox.

Mathematical modeling studies have also been performed as the resolution of the drift paradox. Anholt [20] used a simulation model and argued that, instead of upstream-biased migration, density dependence was an essential factor in population persistence. (Density dependence: areas subjected to greater losses from the drift will experience a higher rate of population increase.) However, his simulation results overly emphasized the effect of density dependence for persistence and ignored the extinction that appeared in the simulations [11]. Humphries and Ruxton [12] changed the way of parameterizing of the principal in Anholt's model and showed that small-scale, random undirected movements on the streambed are enough to explain persistence of drifting organisms. Speirs and Gurney [11] focused on the role of diffusive dispersal as the balancing mechanism for the downstream drift and constructed a simplified one-dimensional model with diffusion. 
Their model is given by

$$
\frac{\partial n}{\partial t}=f(n) n-v \frac{\partial n}{\partial x}+D \frac{\partial^{2} n}{\partial x^{2}} .
$$

This model described a population residing in a stream, a river or an estuary subject to advection and diffusion. Here, $n(x, t)$ is the density of the population per unit area, $f(n)$ is the local per capita growth rate of the population, $v$ is the advection velocity and $D$ is the diffusion coefficient. The advection term represents downstream drift and the diffusive term represents random movement. They also applied the analytic results to four hydrodynamically disparate systems and demonstrated that upstream diffusive movement balanced out downstream flow for persistence.

The importance of involving diffusion is that random motion, due both to turbulent water movement and to randomly directed movements by individual organisms, is a key element in streams and rivers, and also a potential source of upstream recolonization.

Based on Speirs' model, Pachepskey [23] divided the population into two interacting compartments: individuals residing on the benthos (the bottom of the stream) and individuals drifting in the flow. They considered a population in which individuals live and reproduce on the benthos, and occasionally enter the water column to drift until they settle on the benthos again.

The importance of compartmentalization is that aquatic insect larvae spend a considerable proportion of their time immobile on the benthos [21]. For example, zebra mussels have two life stages: a free floating larval stage and an attached adult stage. Larvae are planktonic for 2-4 weeks and adults are sessile. They spend $2 \%$ of their lifetime in the water column. Moreover, the rates that insects switch between benthos and drift can be set by insect behavior rather than by stream hydrodynamics. For instance, some experiments showed that the entry rate to the 
drift can depend on the organisms response to environmental factors such as food abundance and density dependence $[1,21,22]$, and the settling rate to the benthos can be approximately constant for some species [17].

Pachepskey's model was given by

$$
\begin{aligned}
\frac{\partial n_{d}}{\partial t} & =D \frac{\partial^{2} n_{d}}{\partial x^{2}}-v \frac{\partial n_{d}}{\partial x}+\mu n_{b}-\sigma n_{d} \\
\frac{\partial n_{b}}{\partial t} & =f\left(n_{b}\right) n_{b}-\mu n_{b}+\sigma n_{d}
\end{aligned}
$$

where $n_{d}$ is the population density in the drift; $n_{b}$ is the population density on the benthos; $\mu$ is the per capita rate at which individuals in the benthic population enter the drift; $\sigma$ is the per capita rate at which the insects return to the benthos from the stream. In their work, they gave the critical domain size necessary for population persistence, showed the positive effect of the stationary component on the ability of the population to persist and spread by analyzing the propagation speed of a population with two compartments. Some numerical results include:

1. The persistence of the population is guaranteed if, at low population densities, the local growth rate of the stationary component of the population exceeds the rate of entry of individuals into the drift;

2. Persistence and ability to spread are closely connected: if the population cannot advance upstream against the flow, it also cannot persist on any finite spatial domain;

3. Residence in the immobile state always enhances population persistence.

However, Pachepskey did not consider stage structure and time-delay for the individuals to reach maturity from when they were born. A large number of papers have been written on modeling single-species population growth with various life stages using discrete models [25, 26, 27], continuous models [28, 29, 30, 31, 32, 33, 34], and stochastic models [35]. Aiello [24] developed a single-species 
growth model with stage structure consisting of immature and mature stages using a discrete time delay from birth to maturity. They showed that there exists a globally asymptotically stable positive equilibrium under certain conditions and analytically and numerically addressed oscillation properties of solutions with timedelay being considered. But they do not consider multiple species with competition and existence of traveling wave solutions.

In this dissertation I construct a stage-structured delayed reaction-diffusion model with competition between two species in a stream. This model has the benefit of synthesizing and generalizing many of the aforementioned models, while providing a clear focus on persistence of two species in a water flow.

In the construction of our system we allow different time delays for both species from birth to maturity and compartmentalization. We include a classical Lotka-Volterra type term which describes the interspecific competition between the two different species. We also involve two stages of life history, immature and mature, for both species.

In this chapter we aim to give the reader a brief but sufficient introduction on reaction-diffusion equations and delay differential equations so that the core content and results of this dissertation are accessible. Section 1.2 introduces the concept of a reaction-diffusion equation and main results of spreading speeds and traveling wave solutions. Section 1.3 introduces delay differential equations and the typical methodology employed to analyze and study them.

\subsection{Reaction-Diffusion Equations}

Reaction-diffusion equations model the movement of many individuals in an environment or media and a local reaction kinetics such as birth, death, and other reaction process. Reaction-diffusion systems can explain the effects of 
the size, shape, and heterogeneity of the spatial environment on the persistence of species and the structure of communities in ecology. Reaction $(R(u))$ and diffusion (diffusion coefficient $D$ ) both contribute to the interesting dynamical behavior of the solutions of the equation. We may describe the above mechanism by the classical FKPP (Fisher's equation) reaction-diffusion equation [36, 37]

$$
\frac{\partial u}{\partial t}=D \frac{\partial^{2} u}{\partial x^{2}}+R(u)
$$

where $R(u)=u(1-u)$.

Diffusion can be considered as a random walk which starts at a point and takes steps in a random direction. Fick's laws can be used to solve for the diffusion coefficient, $D$, and it addresses that the diffusive flux goes from regions of high concentration to regions of low concentration. A special solution of the equation (1.3) is a propagating front (also called traveling wave solution), separating two non-equilibrium homogeneous states, one of which $(u=1)$ is stable and another one $(\mathrm{u}=0)$ is unstable $[38,39,40]$. Figure 1.1 depicts a numerical solution of the FKPP equation (1.3) for six different time moments $t=0,100,200,400,600,800$ with initial condition $u(x, 0)=f(x)=.05 e^{-5 x^{2}}$.

We notice, from Figure 1.1, that a small local initial fluctuation around $u=$ 0 leads to an instability, and that develops in a nonlinear way: a front propagates away from the initial perturbation. Finally, the uniformly stable state of $u=1$ is established on the whole space interval.

In the real world, there are many cases that species live in the media where the diffusion moves to a certain direction. For example, the seeds flow in the wind and organisms drift in the water flow. When one direction in the random walk is favored, an equation with a first order derivative is the result:

$$
\frac{\partial u}{\partial t}=D \frac{\partial^{2} u}{\partial x^{2}}-v \frac{\partial u}{\partial x}+R(u)
$$




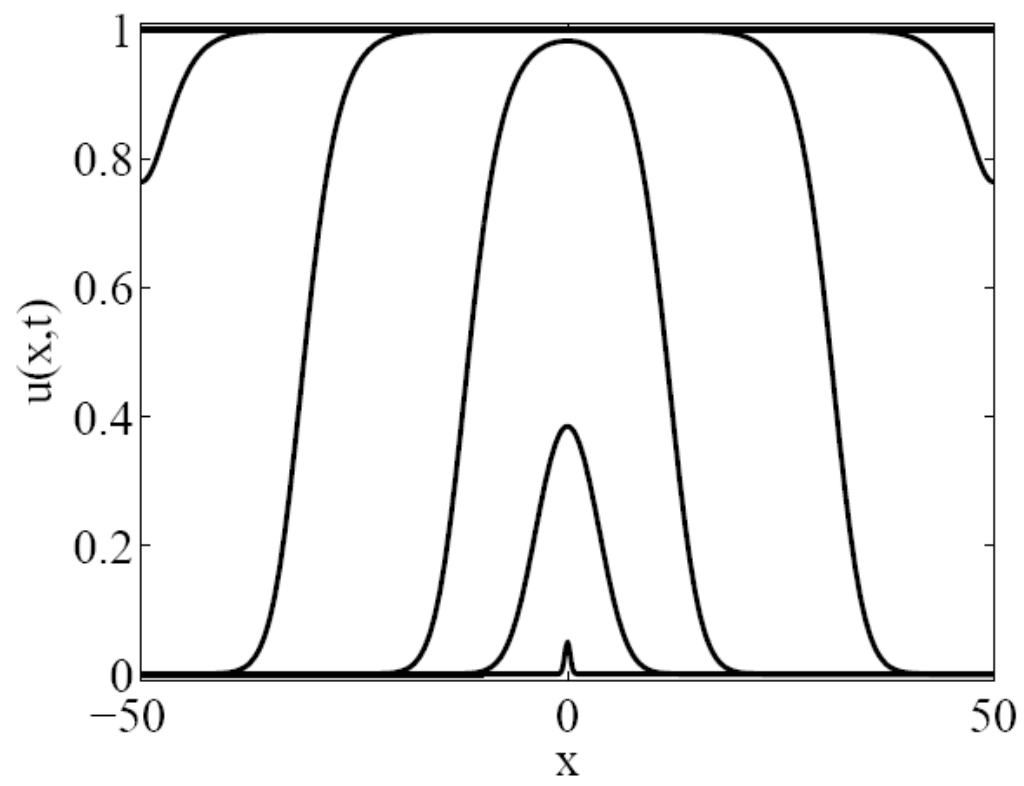

FIGURE 1.1-Traveling wave solutions of FKPP equation, $\frac{\partial u}{\partial t}=D \frac{\partial^{2} u}{\partial x^{2}}+R(u)$, where $R=u-u^{2}$

The fist order derivative term on the right hand side of the equation (1.4) is called an advection term, and the equation (1.4) is called a reaction-diffusion equation with advection.

\subsection{Delay Differential Equations}

Delay differential equations (DDEs) are a type of differential equation in which the derivative of the unknown function at a certain time is given in terms of the values of the function at previous times. We use DDEs because many of the processes in the fields including biology, medicine, chemistry, physics, engineering, and economics involve time delays. For example, in physical processes acceleration and deceleration take little time compared to the times needed to travel most distances, and in biological processes maturation time durations can be large compared to the data-collection times in most population studies. One simple example of DDEs is the Hutchinson equation: 


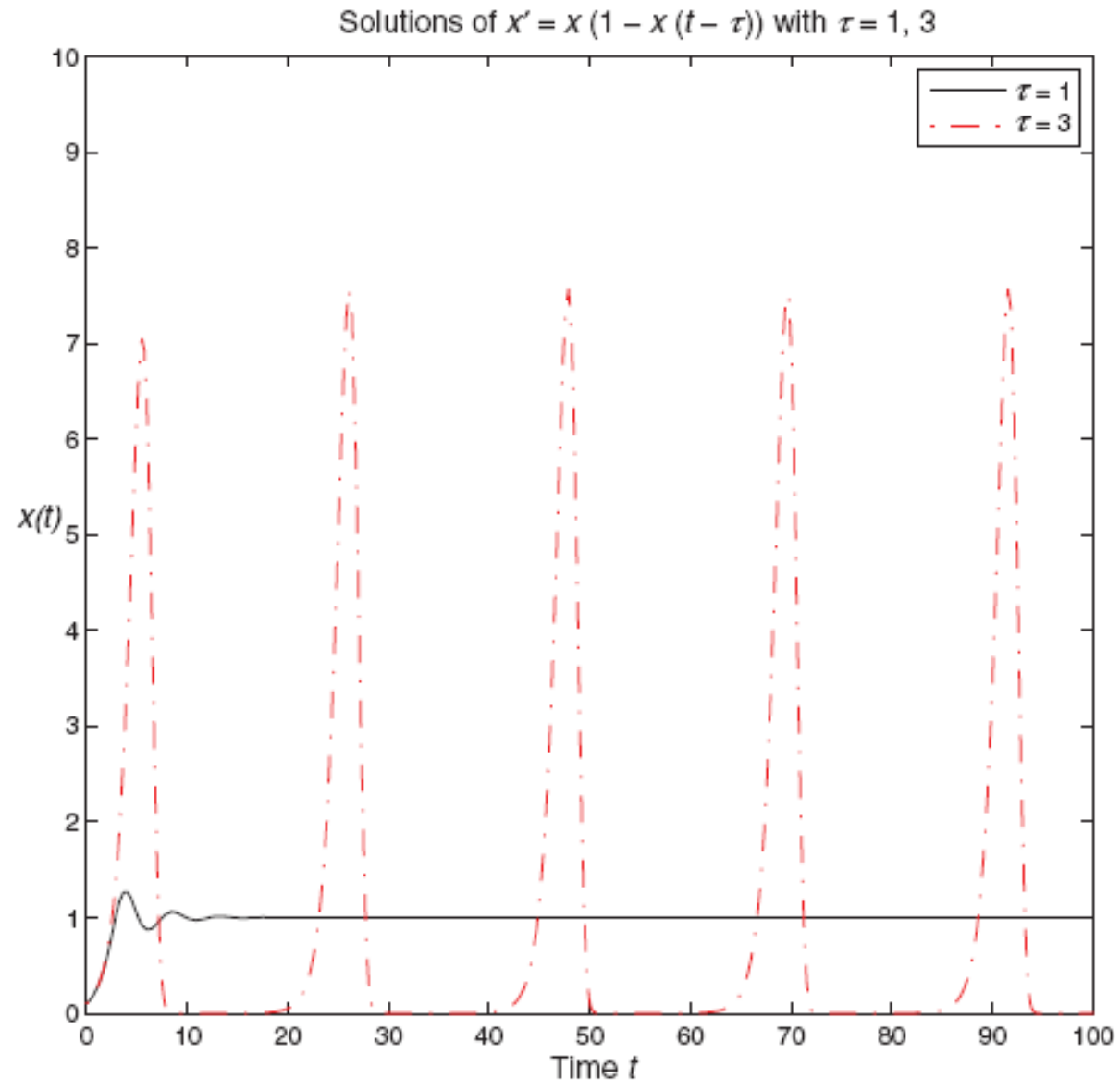

FIGURE 1.2-Solutions of the Hutchinson equation, $\frac{d x(t)}{d t}=r x(1-x(t-\tau) / K)$, where $\tau=1,3$

$$
\frac{d x(t)}{d t}=r x(1-x(t-\tau) / K)
$$

The dynamics of DDEs is more complex than that of ordinary differential equations (ODEs). Figure 1.2 shows solutions of the Hutchinson equation with delays $\tau=1$ and 3, respectively, and gives complex dynamics such as excessive volatility and huge peak-to-valley ratios [43].

DDEs are mostly solved using the "method of steps" given initial condition $\phi:[-\tau, 0] \rightarrow \mathbb{R}^{n}$. Then the solution on the interval $[0, \tau]$ is obtained and is the solution to the inhomogeneous initial value problem 


$$
\begin{aligned}
& \frac{d u(t)}{d t}=-u(t-\tau), \tau>0 \\
& u(t)=\phi(t), t \in[-\tau, 0] .
\end{aligned}
$$

This can be continued for the successive intervals by using the solution to the previous interval as an inhomogeneous term. In practice, the initial value problem is often solved numerically. Moreover, the existence of solutions of the initial value problem has been given in Theorem 1.1.

THEOREM 1.1. Let $f, f_{x} \in \mathcal{C}\left(\mathbb{R}^{3}\right), s \in \mathbb{R}$ and let $\phi:[s-\tau, s] \rightarrow \mathbb{R}$ be a continuous function. Then there exists $\sigma>s$ and a unique solution of the initial value problem

$$
\begin{aligned}
& \frac{d u(t)}{d t}=f(t, x(t), x(t-\tau)), \tau>0 \\
& u(t)=\phi(t), t \in[s-\tau, s] .
\end{aligned}
$$

on $[s-\tau, \sigma]$.

Existence of solutions of delayed reaction-diffusion systems was established by Martin and Smith [45].

Similar to ODEs, many properties including stability of linear DDEs can be characterized and analyzed using the characteristic equation. The characteristic equation associated with the linear DDE with discrete delays

$$
\frac{d x(t)}{d t}=A_{0} x(t)+A_{1} x\left(t-\tau_{1}\right)+\ldots+A_{n} x\left(t-\tau_{n}\right)
$$

is

$$
\operatorname{det}\left(-\lambda I+A_{0}+A_{1} e^{-\tau_{1} \lambda}+\ldots+A_{n} e^{-\tau_{n} \lambda}\right)=0
$$

Because of the exponential in the characteristic equation, the DDE has infinitely many eigenvalues and thus is more complicated to analyze its properties. There are some studies being done to determine the stability of DDEs [41, 42, 43]. Aiello and Freedman [46] developed a time-delay model of single-species growth with stage structure and showed that under suitable hypotheses there exists a 
globally asymptotically stable positive equilibrium. Zhang et al. [64] studied the stability and travelling waves for a time-delayed population system with stage structure. However, it is known that some delay systems experience a change in stability as the time delay increases, while such systems do not experience such changes, the stability problem has not been solved for many general DDEs. 


\section{The Spreading Speed}

In mathematics, many studies have been done to explain why species persist and even spread against the downstream flow and how quickly a population is advancing or retreating in its environment. As a mechanism to quantify spread we consider the asymptotic behavior of the solution as $x$ approaches infinity, and this is called the spreading speed introduced by Aronson and Weinberger [47, 48, 49] for reaction-diffusion equations. We focus on the spreading speed and traveling wave solutions as resolutions of the drift paradox.

For the classical reaction-diffusion equation (1.3) we define the spreading speed $c^{*}$ in the following sense:

1. If $0 \leq u(x, 0)<1$ and $u(x, 0) \equiv 0$ for all sufficiently large $x$, then for any positive $\epsilon$

$$
\lim _{x \rightarrow \infty}\left\{\max _{|x| \geq\left(c^{*}+\epsilon\right) t} u(x, t)\right\}=0 .
$$

2. For every positive number $\sigma$ there exists a positive number $r_{\sigma}$ such that if $0 \leq u(x, 0) \leq 1$, and if $u(x, 0) \geq \sigma$ on an interval of length $r_{\sigma}$, then for any positive $\epsilon$,

$$
\lim _{x \rightarrow \infty}\left\{\sup _{|x| \leq\left(c^{*}-\epsilon\right) t}(1-u(x, t))\right\}=0 .
$$

The first statement says that $c^{*}$ is an upper bound for the spreading speed. If $c^{*}$ is the asymptotic rate of spread, then we would expect that at time $t$, the support of the solution would have grown by $c^{*} t$. Thus, points outside the expected support of the solution $\left(|x| \geq\left(c^{*}+\epsilon\right) t\right)$ should not have individuals present. In other words, if we always move faster than the rate of spread of the population, we should always be in front of the advancing population.

Alternatively, the second statement says that $c^{*}$ is a lower bound for the spreading speed. If we always move more slowly than the population $\left(|x| \leq\left(c^{*}-\right.\right.$ $\epsilon) t$ ) then we should always be behind the leading edge of the advancing population. 


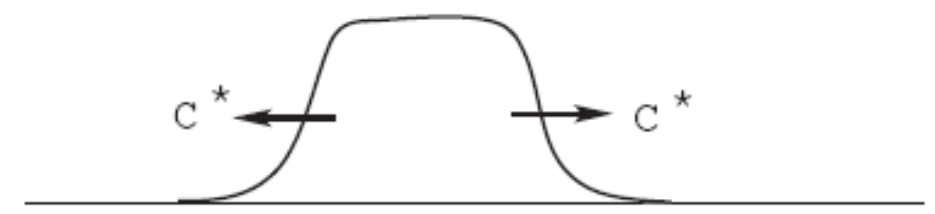

FIGURE 1.3-Spread of the solution of FKPP equation with the speed of $c^{*}=$ $2 \sqrt{D R^{\prime}(0)}$

In this case, the population will approach the steady-state, 1, over a long period of time.

Connecting the first and second statements we conclude that $c^{*}$ is the asymptotic rate of spread of the solutions of the reaction-diffusion equation (1.3).

Aronson and Weinberger $[48,49]$ proved that the spreading speed of FKPP equation (1.3) is given by $c^{*}=2 \sqrt{D R^{\prime}(0)}$ and the compact initial data expands at speed $c^{*}$.

The formula $c^{*}=2 \sqrt{D R^{\prime}(0)}$ can be obtained a similar formula through an abstract operator Q, the time one solution map of (1.3),

$$
u(x, t)=\mathrm{Q}\left[u_{0}\right](x), u_{0}=u(0, x)
$$

and its linearization map M defined by

$$
\mathrm{M}[u](x)=\int_{-\infty}^{\infty} u(x-y) m(y) d y,
$$

where $u$ is a vector of population distributions of species, Q is the time one solution map which models the growth, interaction, and migration of the species, and $m(y)=R^{\prime}(0) \frac{1}{2 \sqrt{\pi D}} e^{-\frac{|y|^{2}}{4 D}}$ is a nonnegative bounded measure [51]. In [51] Weinberger also provided the formula of the spreading speed $c^{*}$ for the reaction-diffusion equation with advection (1.4), $c^{*}=v+2 \sqrt{D R^{\prime}(0)}$. 
For a nonlinear multi-species reaction-diffusion system, there are multiple spreading speeds. The slowest spreading speed $c^{*}$ and the fastest spreading speed $c_{+}^{*}$ are given by Weinberger et al. [52] and Li et al. [61]. An upper bound of all spreading speeds $c_{+}^{*}$ is also given in [52]. Moreover, if the nonlinear system is dominated by a linear system in the direction of the vector corresponding to the principal eigenvalue of the generating matrix, then the nonlinear system has a unique spreading speed (i.e. $c^{*}=c_{+}^{*}$ ), which is equal to that of the linear system [52]. This is known as linear determinacy. Li [53] studied partially degenerate cooperative reaction-diffusion systems, and the result can be applied on Pachepskey's model (1.2).

In the next section we will discuss how the spreading speed is related to traveling wave speeds. 


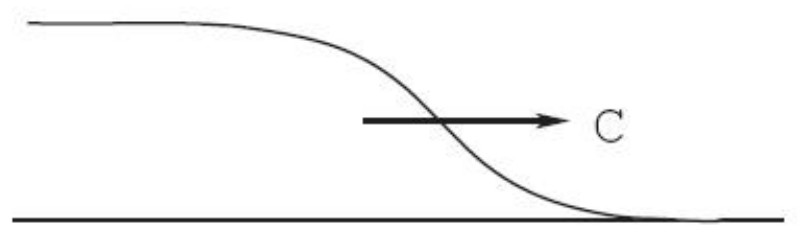

FIGURE 1.4-Traveling wave solution of the reaction-diffusion equation $u(x, t)=$ $w(x-c t)$ with $w(-\infty)=1$ and $w(+\infty)=0$ exists if and only if $c \geq c^{*}$.

\section{Traveling Wave Solutions}

We consider the continuous-time problems such as the reaction-diffusion system

$$
\begin{aligned}
& \mathbf{u}_{, t}=D \mathbf{u}_{, x x}-E \mathbf{u}_{, x}+\mathbf{f}(\mathbf{u}), \\
& \mathbf{u}(0, x)=\mathbf{u}_{0}(x)
\end{aligned}
$$

where $D:=\operatorname{diag}\left(d_{1}, d_{2}, \ldots, d_{k}\right)$ and $E:=\operatorname{diag}\left(e_{1}, e_{2}, \ldots, e_{k}\right)$ are constant diagonal matrices. A traveling wave of the reaction-diffusion system (1.11) with speed $c$ is a solution in the form of $\mathbf{W}(x-c t)$, where $c$ is a positive constant.

Note that traveling wave solutions do not explicitly depend on time. Indeed, they are solutions retaining their shapes which are translated by a fixed length for each iteration of time. Thus the solutions travel in space at the rate of $c$ over time.

There have been a number of investigations of traveling wave solutions and asymptotic behavior in terms of spreading speeds for various evolution systems including nonlinear reaction-diffusion systems $[54,55,56]$ and time-delayed reactiondiffusion systems $[57,58,59,62]$. Weinberger, Lewis and $\operatorname{Li}[52,60,61]$ developed the theory of spreading speeds and monotone traveling wave solutions for cooperative or competition models. For the reaction-diffusion equations (1.11) without the advection term, it has been proved that the spreading speed $c^{*}$ can be characterized as the slowest speed of a class of traveling wave solutions when all the diffusion coefficients $d_{i}>0$ [61]. Furthermore, the above statement holds if all the linear determinacy conditions are satisfied when some but not all $d_{i}=0 \quad[53]$. 
Under appropriate assumptions, when $c \geq c^{*}$ there exists a traveling wave solution $w(x-c t)$ which is non-increasing, and when $c<c^{*}$ there is no traveling wave solution with speed $c$.

For a delayed Lotka-Volterra type competition model, Al-Omari and Gourley [63] showed that for $c \geq \tilde{c}$, where $\tilde{c}$ is some number, the system has a nondecreasing traveling wave solution connecting two mono-culture equilibria. Liang and Zhao [67] discussed the existence of spreading speed and traveling wave solutions for general delayed systems. Recently, Li and Zhang [62] proved the existence of traveling wave solutions that connect a mono-culture equilibrium and a different mono-culture equilibrium.

The connection between spreading speeds and traveling wave speeds is significant because it is often more accessible to calculate the minimum speed of traveling waves than it is to calculate the spreading speed. 


\section{CHAPTER 2 THE MODEL}

In this chapter we introduce a stage-structured delayed reaction-diffusion model for competition between two species in a stream. The construction of our model can be divided into two steps:

\section{The Delayed Reaction-Diffusion Equations For Single Species With}

Stage Structure: Construction of the delayed reaction-diffusion equations is used to describe the growth processes of a single species with advection, diffusion, and stage structure in a stream.

\section{The Lotka-Volterra-type Competition Model Between Two Species:}

We introduce another species with similar growth processes while considering the Lotka-Volterra-type competition between the adult members to describe the interaction between two species in a stream subjected to advection and diffusion.

\subsection{Delayed Reaction-Diffusion Equations For Single Species With Stage Structure}

We begin with an extension of Pachepskey's model (1.2) as a delayed reactiondiffusion two-patch model with age structure. In our single species model, there are two interacting compartments: individuals residing on the benthos and individuals drifting in the flow, as was stated in Pachepskey's model. Individuals live and reproduce on the benthos, and occationally enter the water column to drift 
until they settle on the bethos again. We assume that only adult members on the benthos could switch themself between two compartments and the immature individuals always stay on the stationary compartment until they grow to maturity during a period of time. Therefore, we describe our assumption by introducing the stage structure among the population on the benthos and using $n_{b i}$ and $n_{b m}$ to represent the population density of immature members and the population density of mature members on the benthos, respectively. Moreover, we incorporates a time delay which represents the time from birth to maturity of the benthic population. Before giving our model for single species, we make the following assumptions:

1. Assume that the rate of drift entry is constant, but the model we present is formulated in a way that allows us to easily modify and improve these factors in future work.

2. Assume that (a) transfer between mobile and stationary compartments is via a Poisson process, (b) individual movement can be described as a combination of advection corresponding to the one-dimensional medium with a unidirectional flow as experienced by the organisms and diffusion corresponding to the heterogeneous stream flow and individual swimming, and (c) reproduction occurs on a local scale, i.e. adult members lay eggs where they emerge. These assumptions are intuitively reasonable and have been used in plant population models as well [23, 64].

Our delayed reaction-diffusion two-compartment model with age structure for one species is given by: 


$$
\begin{aligned}
\frac{\partial n_{d}}{\partial t} & =d \frac{\partial^{2} n_{d}}{\partial x^{2}}-e \frac{\partial n_{d}}{\partial x}+\delta n_{b m}-\sigma n_{d} \\
\frac{\partial n_{b i}}{\partial t} & =r n_{b m}-\beta n_{b i}-r e^{-\beta \tau} n_{b m}(t-\tau, x) \\
\frac{\partial n_{b m}}{\partial t} & =r e^{-\beta \tau} n_{b m}(t-\tau, x)-\frac{r\left(n_{b m}\right)^{2}}{\kappa}-\delta n_{b m}+\sigma n_{d} .
\end{aligned}
$$

Here, the last term on the right hand side of the second equation represents the individuals on the benthos born at time $t-\tau$ that are still alive at time $t$ and have reached maturity and arrived at $x$. The death rate of the mature on the benthos is modelled by quadratic term, as in the logistic equation.

Table 2.1 gives a description of each parameter mentioned above.

\subsection{Two Species Lotka-Volterra-type Competition Model}

We next introduce another species with similar growth processes. In order to study how two competing species can persist in streams for some common and limited resource, we use the Lotka-Volterra formulation and add an additional term for each species to account for the species' interactions. We assume that the competition only occurs between the benthic adult individuals and all interactions must be harmful. Our Lotka-Volterra-type competition model is given by:

$$
\begin{aligned}
& \frac{\partial n_{d}^{(1)}}{\partial t}=d_{1} \frac{\partial^{2} n_{d}^{(1)}}{\partial x^{2}}-e_{1} \frac{\partial n_{d}^{(1)}}{\partial x}+\delta_{1} n_{b m}^{(1)}-\sigma_{1} n_{d}^{(1)} \\
& \frac{\partial n_{b i}^{(1)}}{\partial t}=r_{1} n_{b m}^{(1)}-\beta_{1} n_{b i}^{(1)}-r_{1} e^{-\beta_{1} \tau_{1}} n_{b m}^{(1)}\left(t-\tau_{1}, x\right) \\
& \frac{\partial n_{b m}^{(1)}}{\partial t}=r_{1} e^{-\beta_{1} \tau_{1}} n_{b m}^{(1)}\left(t-\tau_{1}, x\right)-\frac{r_{1}\left(n_{b m}^{(1)}\right)^{2}}{\kappa_{1}}-\delta_{1} n_{b m}^{(1)}+\sigma_{1} n_{d}^{(1)}-c_{1} n_{b m}^{(1)} n_{b m}^{(2)} \\
& \frac{\partial n_{d}^{(2)}}{\partial t}=d_{2} \frac{\partial^{2} n_{d}^{(2)}}{\partial x^{2}}-e_{2} \frac{\partial n_{d}^{(2)}}{\partial x}+\delta_{2} n_{b m}^{(2)}-\sigma_{2} n_{d}^{(2)} \\
& \frac{\partial n_{b i}^{(2)}}{\partial t}=r_{2} n_{b m}^{(2)}-\beta_{2} n_{b i}^{(2)}-r_{2} e^{-\beta_{2} \tau_{2}} n_{b m}^{(2)}\left(t-\tau_{2}, x\right) \\
& \frac{\partial n_{b m}^{(2)}}{\partial t}=r_{2} e^{-\beta_{2} \tau_{2}} n_{b m}^{(2)}\left(t-\tau_{2}, x\right)-\frac{r_{2}\left(n_{b m}^{(2)}\right)^{2}}{\kappa_{2}}-\delta_{2} n_{b m}^{(2)}+\sigma_{2} n_{d}^{(2)}-c_{2} n_{b m}^{(1)} n_{b m}^{(2)},
\end{aligned}
$$

where all the parameters have the same descriptions as those in Table 2.1 with

the indices $i=1,2$ indicating the first and second species, respectively, that we consider. As we mentioned at the beginning of this section, we include an addi- 
TABLE 2.1

One Species Model - Parameter Descriptions

\begin{tabular}{ll}
\hline \hline Parameter & Description of Parameter \\
\hline$n_{d}(x, t)$ & population density in the drift at time $t$ and point $x$ \\
\hline$n_{b i}(x, t)$ & $\begin{array}{l}\text { population density of immature members on the benthos at time } \\
t \text { and point } x\end{array}$ \\
\hline$n_{b m}(x, t)$ & $\begin{array}{l}\text { population density of mature members on the benthos at time } t \\
\text { and point } x\end{array}$ \\
\hline$d$ & diffusion coefficient \\
\hline$\delta$ & advection speed experienced by the organisms \\
\hline$\sigma$ & rate at which adult individuals on the benthos enter the drift \\
\hline$r$ & rate at which adult individuals return to the benthos from drift- \\
\hline$\beta$ & birth rate of the population \\
\hline$\tau$ & death rate of the population \\
\hline$\kappa$ & time delay from birth to maturity \\
\hline
\end{tabular}


TABLE 2.2

Simplified Competition Model - Simplified Parameter Descriptions

Parameter Description of Parameter

\begin{tabular}{ll}
\hline$n_{b}^{(i)}$ & population density of adult members of the $i$ th species on the \\
& benthos at time $t$ and point $x, i=1,2$ \\
\hline$r_{i}$ & rate combining two factors: the per capita birth rate and the \\
& survival rate of the immature for the $i$ th species during the im- \\
& mature stage, $i=1,2$
\end{tabular}

tional term for each species to account for their competitions. The parameter $c_{1}$ represents the effect the second species has on the mature of the first species and $c_{2}$ represents the effect the first species has on the mature of the second species. Also, the definition of a competitive Lotka-Volterra system assumes that all $c$-values are positive.

Note that the third and sixth equations in system (2.2) are uncoupled from the second and fifth equations, it is sufficient to consider a simplified system with the second and fifth equations merged:

$$
\begin{aligned}
& \frac{\partial n_{d}^{(1)}}{\partial t}=d_{1} \frac{\partial^{2} n_{d}^{(1)}}{\partial x^{2}}-e_{1} \frac{\partial n_{d}^{(1)}}{\partial x}+\delta_{1} n_{b}^{(1)}-\sigma_{1} n_{d}^{(1)} \\
& \frac{\partial n_{b}^{(1)}}{\partial t}=r_{1} n_{b}^{(1)}\left(t-\tau_{1}, x\right)-\frac{r_{1}\left(n_{b}^{(1)}\right)^{2}}{\kappa_{1}}-\delta_{1} n_{b}^{(1)}+\sigma_{1} n_{d}^{(1)}-c_{1} n_{b}^{(1)} n_{b}^{(2)} \\
& \frac{\partial n_{d}^{(2)}}{\partial t}=d_{2} \frac{\partial^{2} n_{d}^{(2)}}{\partial x^{2}}-e_{2} \frac{\partial n_{d}^{(2)}}{\partial x}+\delta_{2} n_{b}^{(2)}-\sigma_{2} n_{d}^{(2)} \\
& \frac{\partial n_{b}^{(2)}}{\partial t}=r_{2} n_{b}^{(2)}\left(t-\tau_{2}, x\right)-\frac{r_{2}\left(n_{b}^{(2)}\right)^{2}}{\kappa_{2}}-\delta_{2} n_{b}^{(2)}+\sigma_{2} n_{d}^{(2)}-c_{2} n_{b}^{(1)} n_{b}^{(2)}
\end{aligned}
$$

Some new parameters appear in our simplified model (2.3). A description of these parameters can be found in Table 2.2.

In next chapters, we study the system (2.3) analytically and numerically. Before that, we summarize the importance of our extension and difficulties of the 
model study as follows:

\section{Importance of Our Extension}

1. Many biological species reproduce themselves, but it takes some time for the subsequent generation to mature and reproduce.

2. Competition both within and between species is an important topic in ecology, especially community ecology. Competition is one of many interacting biotic and abiotic factors that affect community structure.

\section{Difficulties of The Model Study}

1. The system with delay differential equations is hard to analyze its global stability using general methods.

2. Since each species has two compartments, we have four equations including two DDEs, which increases the difficulty of the proof of existence of traveling wave solutions and the formulation of the spreading speed. 


\section{CHAPTER 3 NONSPATIAL SYSTEM ANALYSIS}

In this chapter we consider only the nonspatial system which models the growth and dispersal of two stage-structured interactive species without the effect of spatial advection and diffusion. We demonstrate that the global stability of the equilibria in the model can be completely determined. The mathematical analysis involves using the fluctuation lemma and constructing sequences approaching equilibrium points.

\subsection{Nonspatial System}

Based on the model (2.3), the corresponding nonspatial system is yield:

$$
\begin{aligned}
& \frac{\partial n_{d}^{(1)}}{\partial t}=\delta_{1} n_{b}^{(1)}-\sigma_{1} n_{d}^{(1)} \\
& \frac{\partial n_{b}^{(1)}}{\partial t}=r_{1} n_{b}^{(1)}\left(t-\tau_{1}, x\right)-\frac{r_{1}\left(n_{b}^{(1)}\right)^{2}}{\kappa_{1}}-\delta_{1} n_{b}^{(1)}+\sigma_{1} n_{d}^{(1)}-c_{1} n_{b}^{(1)} n_{b}^{(2)} \\
& \frac{\partial n_{d}^{(2)}}{\partial t}=\delta_{2} n_{b}^{(2)}-\sigma_{2} n_{d}^{(2)} \\
& \frac{\partial n_{b}^{(2)}}{\partial t}=r_{2} n_{b}^{(2)}\left(t-\tau_{2}, x\right)-\frac{r_{2}\left(n_{b}^{(2)}\right)^{2}}{\kappa_{2}}-\delta_{2} n_{b}^{(2)}+\sigma_{2} n_{d}^{(2)}-c_{2} n_{b}^{(1)} n_{b}^{(2)}
\end{aligned}
$$

with initial conditions

$$
n_{d}^{(i)}(t), n_{b}^{(i)}(t) \geq 0 \text { for }-\tau_{i} \leq t \leq 0, \text { and } n_{d}^{(i)}(0), n_{b}^{(i)}(0)>0 \text { for } i=1,2 .
$$

It is easy to show that the system (3.1) has the trivial equilibrium $E_{0}=$

$(0,0,0,0)$, the mono-culture equilibria $E_{1}=\left(\frac{\delta_{1} \kappa_{1}}{\sigma_{1}}, \kappa_{1}, 0,0\right)$ and $E_{2}=\left(0,0, \frac{\delta_{2} \kappa_{2}}{\sigma_{2}}, \kappa_{2}\right)$, and the coexistence equilibrium

$$
E^{*}=\left(\frac{\delta_{1} \kappa_{1} r_{2}\left(c_{1} \kappa_{2}-r_{1}\right)}{\left(c_{1} c_{2} \kappa_{1} \kappa_{2}-r_{1} r_{2}\right) \sigma_{1}}, \frac{\kappa_{1} r_{2}\left(c_{1} \kappa_{2}-r_{1}\right)}{c_{1} c_{2} \kappa_{1} \kappa_{2}-r_{1} r_{2}}, \frac{\delta_{2} \kappa_{2} r_{1}\left(c_{2} \kappa_{1}-r_{2}\right)}{\left(c_{1} c_{2} \kappa_{1} \kappa_{2}-r_{1} r_{2}\right) \sigma_{2}}, \frac{\kappa_{2} r_{1}\left(c_{2} \kappa_{1}-r_{2}\right)}{c_{1} c_{2} \kappa_{1} \kappa_{2}-r_{1} r_{2}}\right) .
$$


TABLE 3.1

Summary of Existence and Local Stability Criteria of Equilibria

\begin{tabular}{||c||c||c||}
\hline Equilibrium & Existence Criteria & Stability Criteria \\
\hline$E_{0}$ & always exists & always unstable \\
\hline$E_{1}$ & always exists & $r_{2}<c_{2} \kappa_{1}$ \\
\hline$E_{2}$ & always exists & $r_{1}<c_{1} \kappa_{2}$ \\
\hline$E^{*}$ & $\begin{array}{c}\text { If } c_{1} c_{2} \kappa_{1} \kappa_{2}>r_{1} r_{2}, \\
r_{1}<c_{1} \kappa_{2} \text { and } r_{2}<c_{2} \kappa_{1} \\
\text { If } c_{1} c_{2} \kappa_{1} \kappa_{2}<r_{1} r_{2},\end{array}$ & $\begin{array}{c}\text { The global stability analysis in section 3.3 shows that } \\
E_{1}>c_{1} \kappa_{2} \text { and } r_{2}>c_{2} \kappa_{1}\end{array}$ \\
& if $r_{1}>c_{1} \kappa_{2}$ and $r_{2}>c_{2} \kappa_{1}$.
\end{tabular}

\subsection{Local Stability}

We study local stabilities of the equilibria by analyzing the characteristic equations of the linearized system. Here we sketch an outline of our analysis of local stability criteria. Table 3.1 is the summary of our analytical results.

Outline for Local Stability Criteria:

1. Find the characteristic equations of the linearized system for each equilibrium point;

2. Analyze the local stability when there is no time delay in the system, i.e. when $\tau_{i}=0, i=1,2$;

3. Apply the theorem in [43] to the above analysis and get local stability criteria.

\subsection{Global Stability}

In this section, we shall prove Theorem 3.1 on the global asymptotic stability of the equilibria $E_{1}, E_{2}$, and $E^{*}$. Since positivity implies that the system is 
persistent, by the initial condition (3.2), the persistence of the nonspatial system (3.1) is guaranteed.

Before proceeding, we need the following lemmas.

The following two lemmas are elementary but useful in our proof. These were proven in $[65,66]$.

LEMMA 3.1. (Barbălat Lemma) Let a be a finite number, and $f:[a, \infty) \rightarrow \mathbb{R}$ be $a$ differentiable function. If $\lim _{t \rightarrow \infty} f(t)$ exists (finite) and $f^{\prime}$ is uniformly continuous on $[a, \infty)$, then $\lim _{t \rightarrow \infty} f^{\prime}(t)=0$.

LEMMA 3.2. (Fluctuation Lemma) Let a be a finite number, and $f:[a, \infty) \rightarrow \mathbb{R}$ be a differentiable function. If $\liminf _{t \rightarrow \infty} f(t)<\lim _{\sup _{t \rightarrow \infty}} f(t)$, then there exist sequences $\left\{t_{m}\right\} \uparrow \infty$ and $\left\{s_{m}\right\} \uparrow \infty$ such that $\lim _{m \rightarrow \infty} f\left(t_{m}\right)=\limsup _{t \rightarrow \infty} f(t)$, $f^{\prime}\left(t_{m}\right)=0$, and $\lim _{m \rightarrow \infty} f\left(s_{m}\right)=\liminf _{t \rightarrow \infty} f(t), f^{\prime}\left(s_{m}\right)=0$.

Lemma 3.2 states that for an oscillating function $f$, we can always find two sequences satisfying the above conditions. Using Lemma 3.2, we can study the long-term behavior by knowing what happens at the local minimum and maximum points.

LEMMA 3.3. Consider the following one-species system

$$
\begin{aligned}
& \frac{\partial n_{d}^{(1)}}{\partial t}=\delta_{1} n_{b}^{(1)}-\sigma_{1} n_{d}^{(1)} \\
& \frac{\partial n_{b}^{(1)}}{\partial t}=r_{1} n_{b}^{(1)}\left(t-\tau_{1}, x\right)-\frac{r_{1}\left(n_{b}^{(1)}\right)^{2}}{\kappa_{1}}-\delta_{1} n_{b}^{(1)}+\sigma_{1} n_{d}^{(1)} .
\end{aligned}
$$

Let $\left(n_{d}^{(1)}(t), n_{b}^{(1)}(t)\right)$ be the solution of system (3.3), where $n_{d}^{(1)}(t), n_{b}^{(1)}(t)>0$ for $-\tau_{1} \leq t \leq 0$. Then $\lim _{t \rightarrow \infty}\left(n_{d}^{(1)}(t), n_{b}^{(1)}(t)\right)=\left(\frac{\delta_{1} \kappa_{1}}{\sigma_{1}}, \kappa_{1}\right)$.

Proof. We shall prove the lemma with three cases:

Case i: When $\left(n_{d}^{(1)}(t), n_{b}^{(1)}(t)\right)$ is eventually monotonically decreasing, positivity of solutions indicates that $\left(n_{d}^{(1)}(t), n_{b}^{(1)}(t)\right)$ must approach some limit $\hat{N}_{1} \geq \mathbf{0}$. This limit must be an equilibrium of (3.3) and is therefore either $\mathbf{0}$ or $\left(\frac{\delta_{1} \kappa_{1}}{\sigma_{1}}, \kappa_{1}\right)$. 
The trivial equilibrium $\mathbf{0}$ is ruled out since it is unstable by the local stability analysis in Section 3.2.

Case ii: When $\left(n_{d}^{(1)}(t), n_{b}^{(1)}(t)\right)$ is eventually monotonically increasing, then $n_{b}^{(1)}\left(t-\tau_{1}, x\right) \leq n_{b}^{(1)}(t)$ for some sufficiently large $t$ and all $\tau_{1} \in[0, \tau]$ so that

$$
\frac{\partial n_{b}^{(1)}}{\partial t} \leq r_{1} n_{b}^{(1)}(t, x)-\frac{r_{1}\left(n_{b}^{(1)}\right)^{2}}{\kappa_{1}}-\delta_{1} n_{b}^{(1)}+\sigma_{1} n_{d}^{(1)} .
$$

Hence $\lim _{t \rightarrow \infty}\left(n_{d}^{(1)}(t), n_{b}^{(1)}(t)\right)$ exists and is an positive equilibrium of (3.3), i.e. $\lim _{t \rightarrow \infty}\left(n_{d}^{(1)}(t), n_{b}^{(1)}(t)\right)=\left(\frac{\delta_{1} \kappa_{1}}{\sigma_{1}}, \kappa_{1}\right)$.

Case iii: $\left(n_{d}^{(1)}(t), n_{b}^{(1)}(t)\right)$ is neither eventually monotonically decreasing nor increasing. It means that $n_{d}^{(1)}(t)$ and $n_{b}^{(1)}(t)$ have infinite sequences of local maxima and local minima. Define

$$
\begin{aligned}
& \lim \sup _{t \rightarrow \infty} n_{d}^{(1)}(t)=\overline{n_{d}^{(1)}}, \\
& \liminf \operatorname{si\infty }_{t \rightarrow \infty} n_{d}^{(1)}(t)=\underline{n_{d}^{(1)}}, \\
& \lim \sup _{t \rightarrow \infty} n_{b}^{(1)}(t)=\overline{n_{b}^{(1)}}, \text { and } \\
& \liminf \operatorname{si\infty }_{t \rightarrow \infty} n_{b}^{(1)}(t)=\underline{n_{b}^{(1)}} .
\end{aligned}
$$

By Lemma 3.2, there exist $\left\{t_{1 n}^{(+)}\right\},\left\{t_{1 n}^{(-)}\right\},\left\{s_{1 n}^{(+)}\right\}$, and $\left\{s_{1 n}^{(-)}\right\}$such that

$$
\begin{array}{ll}
n_{d}^{(1)}\left(t_{1 n}^{(+)}\right) \rightarrow \overline{n_{d}^{(1)}} \text { as } t \rightarrow \infty, & n_{d}^{(1)}\left(t_{1 n}^{(-)}\right) \rightarrow \underline{n_{d}^{(1)}} \text { as } t \rightarrow \infty, \\
n_{b}^{(1)}\left(s_{1 n}^{(+)}\right) \rightarrow \overline{n_{b}^{(1)}} \text { as } t \rightarrow \infty, \text { and } & n_{b}^{(1)}\left(s_{1 n}^{(-)}\right) \rightarrow \underline{n_{b}^{(1)}} \text { as } t \rightarrow \infty
\end{array}
$$

where $\left(n_{d}^{(1)}\left(t_{1 n}^{( \pm)}\right)\right)^{\prime}=0$ and $\left(n_{b}^{(1)}\left(s_{1 n}^{( \pm)}\right)\right)^{\prime}=0$.

Since $\delta_{1} \underline{n_{b}^{(1)}} \leq \sigma_{1} \underline{n_{d}^{(1)}} \leq \sigma_{1} \overline{n_{d}^{(1)}} \leq \delta_{1} \overline{n_{b}^{(1)}}$, we have $\sigma_{1} \Delta n_{d}^{(1)} \leq \delta_{1} \Delta n_{b}^{(1)}$, where $\Delta n_{d}^{(1)}=\overline{n_{d}^{(1)}}-\underline{n_{d}^{(1)}}$ and $\Delta n_{b}^{(1)}=\overline{n_{b}^{(1)}}-\underline{n_{b}^{(1)}}$.

Now considering the second equation of system (3.3), we have

$$
0=r_{1} n_{b}^{(1)}\left(s_{1 n}^{( \pm)}-\tau_{1}\right)-\frac{r_{1}\left(n_{b}^{(1)}\left(s_{1 n}^{( \pm)}\right)\right)^{2}}{\kappa_{1}}-\delta_{1} n_{b}^{(1)\left(s_{1 n}^{( \pm)}\right)}+\sigma_{1} n_{d}^{(1)}\left(s_{1 n}^{( \pm)}\right) .
$$

Taking the limit of both sides of the above equation as $t \rightarrow \infty$, then

$$
0=r_{1} \widehat{n_{b}^{(1+)}}-\frac{r_{1}\left({\overline{n_{b}^{(1)}}}^{2}\right.}{\kappa_{1}}-\delta_{1} \overline{n_{b}^{(1)}}+\sigma_{1} \widehat{n_{d}^{(1+)}}
$$


and

$$
0=r_{1} \widehat{n_{b}^{(1-)}}-\frac{r_{1}\left(\underline{n_{b}^{(1)}}\right)^{2}}{\kappa_{1}}-\delta_{1} \underline{n_{b}^{(1)}}+\sigma_{1} \widehat{n_{d}^{(1-)}}
$$

where $\widehat{n_{b}^{(1 \pm)}}:=\lim _{t \rightarrow \infty} n_{b}^{(1)}\left(s_{1 n}^{( \pm)}-\tau_{1}\right)$ and $\widehat{n_{d}^{(1 \pm)}}:=\lim _{t \rightarrow \infty} n_{d}^{(1)}\left(s_{1 n}^{( \pm)}\right)$

Since $\underline{n_{b}^{(1)}} \leq \widehat{n_{b}^{(1 \pm)}} \leq \widehat{n_{b}^{(1)}}$, and $\underline{n_{d}^{(1)}} \leq \widehat{n_{d}^{(1 \pm)}} \leq \widehat{n_{d}^{(1)}}$, then

$$
\begin{aligned}
& r_{1} \overline{n_{b}^{(1)}}+\sigma_{1} \overline{n_{d}^{(1)}}-\frac{r_{1}{\overline{n_{b}^{(1)}}}^{2}}{\kappa_{1}}-\delta_{1} \overline{n_{b}^{(1)}} \geq 0 \\
& r_{1} \underline{n_{b}^{(1)}}+\sigma_{1} \underline{n_{d}^{(1)}}-\frac{r_{1}\left(\frac{n_{b}^{(1)}}{2}\right)^{2}}{\kappa_{1}}-\delta_{1} \underline{n_{b}^{(1)}} \leq 0 .
\end{aligned}
$$

Since $\sigma_{1} \overline{n_{d}^{(1)}} \leq \delta_{1} \overline{n_{b}^{(1)}}$ and $\sigma_{1} \underline{n_{d}^{(1)}} \geq \delta_{1} \underline{n_{b}^{(1)}}$, then

$$
\begin{aligned}
& \overline{n_{b}^{(1)}}\left[r_{1}-\frac{r_{1} \overline{n_{b}^{(1)}}}{\kappa_{1}}\right] \geq 0 \\
& \underline{n_{b}^{(1)}}\left[r_{1}-\frac{r_{1} \frac{n_{b}^{(1)}}{\kappa_{1}}}{\kappa_{1}} \leq 0 .\right.
\end{aligned}
$$

If $\overline{n_{b}^{(1)}} \neq 0, \overline{n_{b}^{(1)}} \leq \kappa_{1}$, and if $\underline{n_{b}^{(1)}} \neq 0, \underline{n_{b}^{(1)}} \geq \kappa_{1}$, then $\overline{n_{b}^{(1)}} \leq \underline{n_{b}^{(1)}}$. Thus $\overline{n_{b}^{(1)}}=\underline{n_{b}^{(1)}}$ and $\Delta n_{b}^{(1)}=0$. Since $0 \leq \sigma_{1} \Delta n_{d}^{(1)} \leq \delta_{1} \Delta n_{b}^{(1)}$, we have $\Delta n_{d}^{(1)}=0$.

We know that $\frac{\partial n_{d}^{(1)}}{\partial t}$ and $\frac{\partial n_{b}^{(1)}}{\partial t}$ are bounded, and that $(0,0)$ is locally unstable. By Persistence Theory, $\underline{n_{d}^{(1)}}, \underline{n_{b}^{(1)}}>0$ and $\lim _{t \rightarrow \infty}\left(n_{d}^{(1)}(t), n_{b}^{(1)}(t)\right)=\left(\frac{\delta_{1} \kappa_{1}}{\sigma_{1}}, \kappa_{1}\right)$. The proof of Lemma (3.3) is complete.

Using a similar argument, we can show that $\lim _{t \rightarrow \infty}\left(n_{d}^{(2)}(t), n_{b}^{(2)}(t)\right)=\left(\frac{\delta_{2} \kappa_{2}}{\sigma_{2}}, \kappa_{2}\right)$.

THEOREM 3.1. Let $E_{1}, E_{2}$ and $E^{*}$ be defined as above, then the following statements are valid:

i. If $r_{1}>c_{1} \kappa_{2}, r_{2}<c_{2} \kappa_{1}$, then the mono-culture equilibrium $E_{1}$ is globally asymptotically stable;

ii. If $r_{1}<c_{1} \kappa_{2}, r_{2}>c_{2} \kappa_{1}$, then the mono-culture equilibrium $E_{2}$ is globally asymptotically stable;

iii. If $r_{1}>c_{1} \kappa_{2}, r_{2}>c_{2} \kappa_{1}$, then the unique coexistence equilibrium $E^{*}$ is globally asymptotically stable. 
The approach of the proof of Theorem 3.1 is mainly to use the comparison principle which is inspired by [63] and the Fluctuation Lemma.

\subsubsection{Global Stability of the mono-culture equilibria $E_{1}$ and $E_{2}$}

We shall prove Theorem 3.1 part (i) on the global stability of the monoculture equilibrium point

$$
E_{1}=\left(\frac{\delta_{1} \kappa_{1}}{\sigma_{1}}, \kappa_{1}, 0,0\right)
$$

of the system (3.1), in the situation when the other boundary equilibrium

$$
E_{2}=\left(0,0, \frac{\delta_{2} \kappa_{2}}{\sigma_{2}}, \kappa_{2}\right)
$$

of the system (3.1) is locally unstable. This means that species one out-competes species two in the environment.

Proof of Theorem 3.1 part (i). Let $N_{1}(t)=\left(n_{d}^{(1)}(t), n_{b}^{(1)}(t)\right), N_{2}(t)=\left(n_{d}^{(2)}(t), n_{b}^{(2)}(t)\right)$, and $N_{B}=\left(\frac{\delta_{1} \kappa_{1}}{\sigma_{1}}, \kappa_{1}\right)$ be vectors.

Denote

$$
\begin{array}{ll}
\lim \sup _{t \rightarrow \infty} N_{1}(t)=\overline{N_{1}}, & \liminf \inf _{t \rightarrow \infty} N_{1}(t)=\underline{N_{1}}, \\
\limsup \sup _{t \rightarrow \infty} N_{2}(t)=\overline{N_{2}}, & \liminf t \rightarrow \infty N_{2}(t)=\underline{N_{2}} .
\end{array}
$$

Clearly, $\overline{N_{1}} \leq N_{B}$. And by Persistence Theory, $N_{2} \geq 0$.

Let $\left(n_{d}^{(2)}(t), n_{b 1}^{(2)}(t)\right)$ be the solution of the following system:

$$
\begin{aligned}
& \frac{\partial n_{d}^{(2)}}{\partial t}=\delta_{2} n_{b 1}^{(2)}-\sigma_{2} n_{d}^{(2)} \\
& \frac{\partial n_{b 1}^{(2)}}{\partial t}=r_{2} n_{b 1}^{(2)}\left(t-\tau_{2}, x\right)-\frac{r_{2}\left(n_{b 1}^{(2)}\right)^{2}}{\kappa_{2}}-\delta_{2} n_{b 1}^{(2)}+\sigma_{2} n_{d}^{(2)} .
\end{aligned}
$$

Then $\lim _{t \rightarrow \infty}\left(n_{d}^{(2)}(t), n_{b 1}^{(2)}(t)\right)=V_{1}$, where $V_{1}=\left(\frac{\delta_{2} \kappa_{2}}{\sigma_{2}}, \kappa_{2}\right)$.

By the Comparison Principle in [44], $\overline{N_{2}} \leq V_{1}$. 
Let $\epsilon>0$ be sufficiently small such that $0<\epsilon<\frac{\kappa_{2}\left(r_{1}-c_{1} \kappa_{2}\right)}{r_{2} c_{1}}$. There exists $t_{1}>\tau_{2}$ such that $N_{2} \leq V_{1}+\left(\begin{array}{l}\varepsilon \\ \epsilon\end{array}\right)$ for all $t \geq t_{1}$ where $\varepsilon>0$ is a sufficiently small number.

Let $\left(n_{d}^{(1)}, n_{b 1}^{(1)}\right)$ satisfy the following system:

$$
\begin{aligned}
& \frac{\partial n_{d}^{(1)}}{\partial t}=\delta_{1} n_{b 1}^{(1)}-\sigma_{1} n_{d}^{(1)} \\
& \frac{\partial n_{b 1}^{(1)}}{\partial t}=r_{1} n_{b 1}^{(1)}\left(t-\tau_{1}, x\right)-\frac{r_{1}\left(n_{b 1}^{(1)}\right)^{2}}{\kappa_{1}}-\delta_{1} n_{b 1}^{(1)}+\sigma_{1} n_{d}^{(1)}-c_{1} n_{b 1}^{(1)}\left(\kappa_{2}+\epsilon\right) .
\end{aligned}
$$

Then $\lim _{t \rightarrow \infty}\left(n_{d}^{(1)}(t), n_{b 1}^{(1)}(t)\right)=U_{1}$, where $U_{1}=\left(\frac{\delta_{1} \kappa_{1}}{\sigma_{1}}, \frac{\kappa_{1}\left[r_{1}-c_{1}\left(\kappa_{2}+\epsilon\right)\right]}{r_{1}}\right)$.

By the positiveness of $\epsilon$ and $\varepsilon$ and Comparison Principle, $N_{1} \geq U_{1}$.

Let $\epsilon, \varepsilon>0$, there exists $t_{2}>0$ such that $N_{1} \geq U_{1}-\left(\begin{array}{l}\varepsilon \\ \epsilon\end{array}\right)$ for all $t>t_{2}$. For $t>t_{2}$, let $\left(n_{d}^{(2)}, n_{b 2}^{(2)}\right)$ satisfy the following system:

$$
\begin{aligned}
& \frac{\partial n_{d}^{(2)}}{\partial t}=\delta_{2} n_{b 2}^{(2)}-\sigma_{2} n_{d}^{(2)} \\
& \frac{\partial n_{b 2}^{(2)}}{\partial t}=r_{2} n_{b 2}^{(2)}\left(t-\tau_{2}, x\right)-\frac{r_{2}\left(n_{b 2}^{(2)}\right)^{2}}{\kappa_{2}}-\delta_{2} n_{b 2}^{(2)}+\sigma_{2} n_{d}^{(2)}-\frac{r_{2}\left(U_{1}^{(2)}-\epsilon\right) n_{b 2}^{(2)}}{\kappa_{1}} .
\end{aligned}
$$

Since $\frac{\partial n_{b}^{(2)}}{\partial t} \leq \frac{\partial n_{b 2}^{(2)}}{\partial t}$, by the arbitrariness of $\epsilon$ and Comparison Principle, $N_{2} \leq$ $\left(n_{d}^{(2)}, n_{b 2}^{(2)}\right)$. Hence $\overline{N_{2}} \leq \lim _{t \rightarrow \infty}\left(n_{d}^{(2)}(t), n_{b 2}^{(2)}(t)\right)=V_{2}$, where $V_{2}=\left(\frac{\delta_{2} \kappa_{2}}{\sigma_{2}}, \kappa_{2}-\frac{\kappa_{2} U_{1}^{(2)}}{\kappa_{1}}\right)$.

Let $\epsilon, \varepsilon>0$, there exists $t_{3}>0$ such that $N_{2} \leq V_{2}+\left(\begin{array}{l}\varepsilon \\ \epsilon\end{array}\right)$ for all $t>t_{3}$. For $t>t_{3}$, let $\left(n_{d}^{(1)}, n_{b 2}^{(1)}\right)$ satisfy the following system:

$$
\begin{aligned}
& \frac{\partial n_{d}^{(1)}}{\partial t}=\delta_{1} n_{b 2}^{(1)}-\sigma_{1} n_{d}^{(1)} \\
& \frac{\partial n_{b 2}^{(1)}}{\partial t}=r_{1} n_{b 2}^{(1)}\left(t-\tau_{1}, x\right)-\frac{r_{1}\left(n_{b 2}^{(1)}\right)^{2}}{\kappa_{1}}-\delta_{1} n_{b 2}^{(1)}+\sigma_{1} n_{d}^{(1)}-c_{1} n_{b 2}^{(1)}\left(V_{2}^{(2)}+\epsilon\right) .
\end{aligned}
$$

Since $\frac{\partial n_{b}^{(1)}}{\partial t} \geq \frac{\partial n_{b 2}^{(1)}}{\partial t}$, by the arbitrariness of $\epsilon$ and Comparison Principle, $N_{1} \geq$ $\left(n_{d}^{(1)}, n_{b 2}^{(1)}\right)$. Hence $\underline{N_{1}} \geq \lim _{t \rightarrow \infty}\left(n_{d}^{(1)}(t), n_{b 2}^{(1)}(t)\right)=U_{2}$, where $U_{2}=\left(\frac{\delta_{1} \kappa_{1}}{\sigma_{1}}, \frac{\kappa_{1}\left[r_{1}-c_{1} V_{2}^{(2)}\right]}{r_{1}}\right)$.

Continuing this process, we obtain two vector sequences $\left\{U_{m}\right\}$ and $\left\{V_{m}\right\}$, $m=1,2,3, \ldots$ such that for $m \geq 2$,

$$
U_{m}=\left(\frac{\delta_{1} \kappa_{1}}{\sigma_{1}}, \frac{\kappa_{1}\left[r_{1}-c_{1} V_{m}^{(2)}\right]}{r_{1}}\right)
$$


and

$$
V_{m}=\left(\frac{\delta_{2} \kappa_{2}}{\sigma_{2}}, \frac{\kappa_{2}\left[r_{2}-\frac{r_{2} U_{m-1}^{(2)}}{\kappa_{1}}\right]}{r_{2}}\right) .
$$

Combining the above two equations, we have

$$
V_{m}=\left(\frac{\delta_{2} \kappa_{2}}{\sigma_{2}}, \frac{\kappa_{2} c_{1} V_{m-1}^{(2)}}{r_{1}}\right)
$$

Since $\frac{\kappa_{2} c_{1}}{r_{1}}<1$

$$
\lim _{m \rightarrow \infty} V_{m}^{(2)}=0
$$

Hence,

$$
\lim _{m \rightarrow \infty} U_{m}=N_{B}
$$

Since the limits of $n_{d}^{(1)}, n_{b}^{(1)}$, and $n_{b}^{(2)}$ exist, and $\lim _{t \rightarrow \infty} n_{b}^{(2)}=0$, then $\lim _{t \rightarrow \infty} n_{d}^{(2)}=0$.

Therefore, $\lim _{t \rightarrow \infty} N_{1}=N_{B}$ and $\lim _{t \rightarrow \infty} N_{2}=\mathbf{0}$. We use the notation 0 for the constant vector all of whose components are 0 . That is,

$$
\lim _{t \rightarrow \infty}\left(n_{d}^{(1)}(t), n_{b}^{(1)}(t), n_{d}^{(2)}(t), n_{b}^{(2)}(t)\right)=\left(\frac{\delta_{1} \kappa_{1}}{\sigma_{1}}, \kappa_{1}, 0,0\right)
$$

which completes the proof of Theorem 3.1 part (i).

Similarly, we can show that when $E_{1}$ is unstable, $E_{2}$ is asymptotically stable. The proof of Theorem 3.1 part (ii) is omitted here.

\subsubsection{Global Stability of the coexistence state $E^{*}$}

In this subsection, we prove Theorem 3.1 part (iii) on the global stability of the coexistence equilibrium

$$
E^{*}=\left(\frac{\delta_{1} \kappa_{1} r_{2}\left(c_{1} \kappa_{2}-r_{1}\right)}{\left(c_{1} c_{2} \kappa_{1} \kappa_{2}-r_{1} r_{2}\right) \sigma_{1}}, \frac{\kappa_{1} r_{2}\left(c_{1} \kappa_{2}-r_{1}\right)}{c_{1} c_{2} \kappa_{1} \kappa_{2}-r_{1} r_{2}}, \frac{\delta_{2} \kappa_{2} r_{1}\left(c_{2} \kappa_{1}-r_{2}\right)}{\left(c_{1} c_{2} \kappa_{1} \kappa_{2}-r_{1} r_{2}\right) \sigma_{2}}, \frac{\kappa_{2} r_{1}\left(c_{2} \kappa_{1}-r_{2}\right)}{c_{1} c_{2} \kappa_{1} \kappa_{2}-r_{1} r_{2}}\right)
$$

of the system (3.1). We show that when both of the mono-culture equilibria $E_{1}$ and $E_{2}$ are linearly unstable, the coexistence equilibrium $E^{*}$ is globally asymptotically stable. 
Proof of Theorem 3.1 part (iii). Remark: The notations of $N_{1}, N_{2}, \overline{N_{1}}, \underline{N_{1}}, \overline{N_{2}}$, and $N_{2}$ are defined as same as those in the proof of part (i). To prove this statement, it is equivalent to show that

$$
\lim _{t \rightarrow \infty} N_{1}=U^{*}
$$

and

$$
\lim _{t \rightarrow \infty} N_{2}=V^{*}
$$

where

$U^{*}=\left(\frac{\delta_{1} \kappa_{1} r_{2}\left(c_{1} \kappa_{2}-r_{1}\right)}{\left(c_{1} c_{2} \kappa_{1} \kappa_{2}-r_{1} r_{2}\right) \sigma_{1}}, \frac{\kappa_{1} r_{2}\left(c_{1} \kappa_{2}-r_{1}\right)}{c_{1} c_{2} \kappa_{1} \kappa_{2}-r_{1} r_{2}}\right), V^{*}=\left(\frac{\delta_{2} \kappa_{2} r_{1}\left(c_{2} \kappa_{1}-r_{2}\right)}{\left(c_{1} c_{2} \kappa_{1} \kappa_{2}-r_{1} r_{2}\right) \sigma_{2}}, \frac{\kappa_{2} r_{1}\left(c_{2} \kappa_{1}-r_{2}\right)}{c_{1} c_{2} \kappa_{1} \kappa_{2}-r_{1} r_{2}}\right)$

when $E_{1}$ and $E_{2}$ are both linearly unstable.

Let $\left\{N_{m}^{u}(t)\right\},\left\{N_{m}^{v}(t)\right\},\left\{M_{m}^{u}(t)\right\}$, and $\left\{M_{m}^{v}(t)\right\}$ be four vector sequences where $m=1,2,3, \ldots$. We shall need the above sequences satisfying that

$$
M_{m}^{u} \leq \underline{N_{1}} \leq \overline{N_{1}} \leq N_{m}^{u}
$$

and

$$
M_{m}^{v} \leq \underline{N_{2}} \leq \overline{N_{2}} \leq N_{m}^{v}
$$

From positivity of solutions we obtain $N_{1}^{u}$ as follows:

$$
\begin{aligned}
& \frac{\partial n_{d}^{(1)}}{\partial t}=\delta_{1} n_{b}^{(1)}-\sigma_{1} n_{d}^{(1)} \\
& \frac{\partial n_{b}^{(1)}}{\partial t} \leq r_{1} n_{b}^{(1)}\left(t-\tau_{1}, x\right)-\frac{r_{1}\left(n_{b}^{(1)}\right)^{2}}{\kappa_{1}}-\delta_{1} n_{b}^{(1)}+\sigma_{1} n_{d}^{(1)} .
\end{aligned}
$$

Hence

$$
\overline{N_{1}} \leq N_{1}^{u}, \text { where } N_{1}^{u}=\left(\frac{\delta_{1} \kappa_{1}}{\sigma_{1}}, \kappa_{1}\right) .
$$

In a similar way, we have

$$
\overline{N_{2}} \leq N_{1}^{v}, \text { where } N_{1}^{v}=\left(\frac{\delta_{2} \kappa_{2}}{\sigma_{2}}, \kappa_{2}\right) .
$$

Let $\epsilon, \varepsilon>0$ be sufficiently small such that $\epsilon<\frac{r_{2}-c_{2} \kappa_{1}}{c_{2}}$. Let $t_{1}>0$ be such that $N_{1}(t) \leq N_{1}^{u}+\left(\begin{array}{l}\varepsilon \\ \epsilon\end{array}\right)$ for all $t \geq t_{1}$. 


$$
\begin{aligned}
& \text { For } t>t_{1} \text {, let } m_{1}^{v}(t):=\left(\begin{array}{c}
m_{1}^{v(1)}(t) \\
m_{1}^{v(2)}(t)
\end{array}\right) \text { be a solution of the following system } \\
& \frac{\partial m_{1}^{v(1)}(t)}{\partial t}=\delta_{2} m_{1}^{v(2)}-\sigma_{2} m_{1}^{v(1)} \\
& \frac{\partial m_{1}^{v(2)}(t)}{\partial t}=r_{2} m_{1}^{v(2)}\left(t-\tau_{2}, x\right)-\frac{r_{2}\left(m_{1}^{v(2)}\right)^{2}}{\kappa_{2}}-\delta_{2} m_{1}^{v(2)}+\sigma_{2} m_{1}^{v(1)}-c_{2}\left(N_{1}^{u(2)}+\epsilon\right) m_{1}^{v(2)},
\end{aligned}
$$

with appropriate initial data on $\left[t_{1}-\tau_{2}, t_{1}\right]$. Then $\lim _{t \rightarrow \infty} m_{1}^{v(2)}=\kappa_{2}-\frac{\kappa_{2} c_{2}\left(N_{1}^{u(2)}+\epsilon\right)}{r_{2}}$.

$$
\begin{aligned}
\text { Since } N_{1}(t) \leq N_{1}^{u}+\left(\begin{array}{l}
\varepsilon \\
\epsilon
\end{array}\right) \text { for } t \geq t_{1} \\
\frac{\partial N_{2}(t)}{\partial t} \geq \frac{\partial m_{1}^{v}(t)}{\partial t} .
\end{aligned}
$$

So $N_{2}(t) \geq m_{1}^{v}(t)$ and hence

$$
\underline{N_{2}} \geq \liminf _{t \rightarrow \infty} m_{1}^{v}(t)=\left(v_{l 1}, \kappa_{2}-\frac{\kappa_{2} c_{2}\left(N_{1}^{u(2)}+\epsilon\right)}{r_{2}}\right), \text { where } v_{l 1}=\liminf _{t \rightarrow \infty} m_{1}^{v(1)}(t) .
$$

By the arbitrariness of $\epsilon$, we have

$$
\underline{N_{2}} \geq M_{1}^{v}, \text { where } M_{1}^{v}=\left(v_{l 1}, \kappa_{2}-\frac{\kappa_{2} c_{2} N_{1}^{u(2)}}{r_{2}}\right)
$$

With similar argument, we can show that

$$
\underline{N_{1}} \geq M_{1}^{u}, \text { where } M_{1}^{u}=\left(u_{l 1}, \kappa_{1}-\frac{\kappa_{1} c_{1} N_{1}^{v(2)}}{r_{1}}\right) \text { and } u_{l 1}=\liminf _{t \rightarrow \infty} m_{1}^{u(1)}(t) .
$$

Let $\epsilon, \varepsilon$ and $t>0$, there exists $t_{2}>0$ such that $N_{2} \geq M_{1}^{v}-\left(\begin{array}{l}\varepsilon \\ \epsilon\end{array}\right)$ for all $t \geq t_{2}$. Then for $t>t_{2}$,

$$
\begin{aligned}
& \frac{\partial n_{d}^{(1)}(t)}{\partial t}=\delta_{1} n_{b}^{(1)}-\sigma_{1} n_{d}^{(1)} \\
& \frac{\partial n_{b}^{(1)}(t)}{\partial t} \leq r_{1} n_{b}^{(1)}\left(t-\tau_{1}, x\right)-\frac{r_{1}\left(n_{b}^{(1)}\right)^{2}}{\kappa_{1}}-\delta_{1} n_{b}^{(1)}+\sigma_{1} n_{d}^{(1)}-c_{1}\left(M_{1}^{v(2)}-\epsilon\right) n_{b}^{(1)} .
\end{aligned}
$$

Denote $n_{2}^{u}(t)=\left(\begin{array}{c}n_{2}^{u(1)}(t) \\ n_{2}^{u(2)}(t)\end{array}\right)$ as the solution of

$$
\begin{aligned}
& \frac{\partial n_{2}^{u(1)}(t)}{\partial t}=\delta_{1} n_{2}^{u(2)}(t)-\sigma_{1} n_{2}^{u(1)}(t) \\
& \frac{\partial n_{2}^{u(2)}(t)}{\partial t}=r_{1} n_{2}^{u(2)}\left(t-\tau_{1}, x\right)-\frac{r_{1}\left(n_{2}^{u(2)}\right)^{2}}{\kappa_{1}}-\delta_{1} n_{2}^{u(2)}+\sigma_{1} n_{2}^{u(1)}-c_{1}\left(M_{1}^{v(2)}-\epsilon\right) n_{2}^{u(2)},
\end{aligned}
$$


for $t>t_{2}$ with appropriate initial data. Then $N_{1}(t) \leq n_{2}^{u}(t)$ and thus

$$
\overline{N_{1}(t)} \leq \limsup _{t \rightarrow \infty} n_{2}^{u}(t)=\left(u_{u 1}, \kappa_{1}-\frac{\kappa_{1} c_{1}\left(M_{1}^{v(2)}-\epsilon\right)}{r_{1}}\right), \text { where } u_{u 1}=\limsup _{t \rightarrow \infty} n_{2}^{u(1)}(t) .
$$

Since $\epsilon>0$ is arbitrary,

$$
\overline{N_{1}(t)} \leq N_{2}^{u}, \text { where } N_{2}^{u}=\left(u_{u 1}, \kappa_{1}-\frac{\kappa_{1} c_{1} M_{1}^{v(2)}}{r_{1}}\right)
$$

Similarly,

$$
\overline{N_{2}(t)} \leq N_{2}^{v} \text {, where } N_{2}^{v}=\left(v_{u 1}, \kappa_{2}-\frac{\kappa_{2} c_{2} M_{1}^{u(2)}}{r_{2}}\right) \text { and } v_{u 1}=\limsup _{t \rightarrow \infty} n_{2}^{v(1)}(t) .
$$

Now one can show that the transition from the $(m-1)$ th to the $m$ th step in this iterative process is given by

$$
\begin{aligned}
& N_{m}^{u(2)}=\kappa_{1}-\frac{\kappa_{1} c_{1} M_{m-1}^{v(2)}}{r_{1}}, \\
& N_{m}^{v(2)}=\kappa_{2}-\frac{\kappa_{2} c_{2} M_{m-1}^{u(2)}}{r_{2}}, \\
& M_{m}^{u(2)}=\kappa_{1}-\frac{\kappa_{1} c_{1} N_{m}^{v(2)}}{r_{1}}, \\
& M_{m}^{v(2)}=\kappa_{2}-\frac{\kappa_{2} c_{2} N_{m}^{u(2)}}{r_{2}} .
\end{aligned}
$$

Of course, $M_{m}^{u} \leq \underline{N_{1}} \leq \overline{N_{1}} \leq N_{m}^{u}$ and $M_{m}^{v} \leq \underline{N_{2}} \leq \overline{N_{2}} \leq N_{m}^{v}$ for each $m=$ $1,2,3, \ldots$

Next we need to show that $M_{m}^{u}$ and $N_{m}^{u}$ both approach $U^{*}$ as $m \rightarrow \infty$ and that $M_{m}^{v}$ and $N_{m}^{v}$ both approach $V^{*}$.

Note that

$$
N_{m}^{u(2)}=\kappa_{1}-\frac{\kappa_{1} \kappa_{2} c_{1}}{r_{1}}+\frac{\kappa_{1} \kappa_{2} c_{1} c_{2} N_{m-1}^{u(2)}}{r_{1} r_{2}}
$$

By the assumption of $r_{1}>c_{1} \kappa_{2}$ and $r_{2}>c_{2} \kappa_{1}$, we see that $\frac{\kappa_{1} \kappa_{2} c_{1} c_{2}}{r_{1} r_{2}}<1$. We claim that $N_{m}^{u(2)}$ is a monotonically decreasing sequence that is bounded below by $U^{*(2)}$. We consider

$$
\begin{aligned}
\frac{N_{m}^{u(2)}}{N_{m-1}^{u(2)}} & =\frac{\kappa_{1} r_{1}-\kappa_{1} \kappa_{2} c_{1}}{r_{1} N_{m-1}^{u(2)}}+\frac{\kappa_{1} \kappa_{2} c_{1} c_{2}}{r_{1} r_{2}} \\
& \leq \frac{\kappa_{1} r_{1}-\kappa_{1} \kappa_{2} c_{1}}{r_{1} U^{*(2)}}+\frac{\kappa_{1} \kappa_{2} c_{1} c_{2}}{r_{1} r_{2}} \\
& =1,
\end{aligned}
$$


so $N_{m}^{u(2)}$ is monotonically decreasing. Hence $N_{m}^{u(2)}$ converges to a limit, which is $U^{*(2)}$

Certainly, convergence of $N_{m}^{u(2)}$ implies convergence of $M_{m}^{v(2)}$, and it is easily checked that $M_{m}^{v(2)}$ has the limit $V^{*(2)}$. The analysis for the remaining two sequences is similar.

Now we have

$$
\lim _{t \rightarrow \infty} n_{b}^{(1)}(t)=\frac{\kappa_{1} r_{2}\left(c_{1} \kappa_{2}-r_{1}\right)}{c_{1} c_{2} \kappa_{1} \kappa_{2}-r_{1} r_{2}}
$$

and

$$
\lim _{t \rightarrow \infty} n_{b}^{(2)}(t)=\frac{\kappa_{2} r_{1}\left(c_{2} \kappa_{1}-r_{2}\right)}{c_{1} c_{2} \kappa_{1} \kappa_{2}-r_{1} r_{2}}
$$

Since $\lim _{t \rightarrow \infty} \frac{\partial n_{d}^{(1)}(t)}{\partial t}=0$ and $\lim _{t \rightarrow \infty} \frac{\partial n_{d}^{(2)}(t)}{\partial t}=0$, we have

$$
\lim _{t \rightarrow \infty} n_{d}^{(1)}(t)=\frac{\delta_{1} \kappa_{1} r_{2}\left(c_{1} \kappa_{2}-r_{1}\right)}{\left(c_{1} c_{2} \kappa_{1} \kappa_{2}-r_{1} r_{2}\right) \sigma_{1}}
$$

and

$$
\lim _{t \rightarrow \infty} n_{d}^{(2)}(t)=\frac{\delta_{2} \kappa_{2} r_{1}\left(c_{2} \kappa_{1}-r_{2}\right)}{\left(c_{1} c_{2} \kappa_{1} \kappa_{2}-r_{1} r_{2}\right) \sigma_{2}} .
$$

The proof of Theorem 3.1 part (iii) is complete. Therefore we complete the proof of Theorem 3.1.

By the study of the local and global stability of the equilibria of the nonspatial system (3.1), we conclude that:

1. At least one of the two interactive species with stage structure can persist in a stream due to the fact that the trivial equilibrium $E_{0}$ is always unstable.

2. One species out-competes the other one. In other words, one of them will die out due to the competition for the limited resource in the long run.

3. However, under the conditions that the two mono-culture equilibria $E_{1}$ and $E_{2}$ are both unstable, the two species can coexist and approach a stable population density in long term. This is the explaination of the fact that the 
unique coexistence equilibrium $E^{*}$ is globally asymptotically stable under certain conditions given in Theorem 3.1 part (iii). 


\section{CHAPTER 4 EXISTENCE OF TRAVELING WAVES}

In this chapter we analyze the spatial system which models spread of two competitive species with stage structure and time delay in a stream subjected to advection and diffusion:

$$
\begin{aligned}
\frac{\partial n_{d}^{(1)}}{\partial t} & =d_{1} \frac{\partial^{2} n_{d}^{(1)}}{\partial x^{2}}-e_{1} \frac{\partial n_{d}^{(1)}}{\partial x}+\delta_{1} n_{b}^{(1)}-\sigma_{1} n_{d}^{(1)} \\
\frac{\partial n_{b}^{(1)}}{\partial t} & =r_{1} n_{b}^{(1)}\left(t-\tau_{1}, x\right)-\frac{r_{1}\left(n_{b}^{(1)}\right)^{2}}{\kappa_{1}}-\delta_{1} n_{b}^{(1)}+\sigma_{1} n_{d}^{(1)}-c_{1} n_{b}^{(1)} n_{b}^{(2)} \\
\frac{\partial n_{d}^{(2)}}{\partial t} & =d_{2} \frac{\partial^{2} n_{d}^{(2)}}{\partial x^{2}}-e_{2} \frac{\partial n_{d}^{(2)}}{\partial x}+\delta_{2} n_{b}^{(2)}-\sigma_{2} n_{d}^{(2)} \\
\frac{\partial n_{b}^{(2)}}{\partial t} & =r_{2} n_{b}^{(2)}\left(t-\tau_{2}, x\right)-\frac{r_{2}\left(n_{b}^{(2)}\right)^{2}}{\kappa_{2}}-\delta_{2} n_{b}^{(2)}+\sigma_{2} n_{d}^{(2)}-c_{2} n_{b}^{(1)} n_{b}^{(2)} .
\end{aligned}
$$

Here $n_{d}^{(i)}(x, t)$ represents the population density of the $i$ th species in the drift at time $t$ and point $x, n_{b}^{(i)}(x, t)$ represents the population density of adult members of the $i$ th benthic species at time $t$ and point $x$, and the description of parameters $d_{i}, e_{i}, \sigma_{i}, \delta_{i}, r_{i}$ and $\beta_{i}$ can be found in Tables 2.1 and 2.2. This is the system (2.3) which was developed in Chapter 2.

In section 4.1 we define the integral system and show that a traveling wave solution of the delayed reaction-diffusion system is equivalent to a fixed point of a compact integral operator. In section 4.2 we prove that via integral systems there exist traveling wave solutions with speeds above two extended real numbers.

\subsection{Integral System}

Assume $E_{1}=\left(\frac{\delta_{1} \kappa_{1}}{\sigma_{1}}, \kappa_{1}, 0,0\right)$ is unstable, i.e., $r_{2}>c_{2} \kappa_{1}$.

Let $n_{d}^{(1)}:=\frac{\delta_{1} \kappa_{1}}{\sigma_{1}}-n_{d}^{(1)}$ and $n_{b}^{(1)}:=\kappa_{1}-n_{b}^{(1)}$. We convert the competition 
system (4.1) into the following cooperative system:

$$
\begin{aligned}
& \frac{\partial n_{d}^{(1)}}{\partial t}=d_{1} \frac{\partial^{2} n_{d}^{(1)}}{\partial x^{2}}-e_{1} \frac{\partial n_{d}^{(1)}}{\partial x}+\delta_{1} n_{b}^{(1)}-\sigma_{1} n_{d}^{(1)} \\
& \frac{\partial n_{b}^{(1)}}{\partial t}=r_{1} n_{b}^{(1)}\left(t-\tau_{1}, x\right)+\frac{r_{1}\left(n_{b}^{(1)}\right)^{2}}{\kappa_{1}}-2 r_{1} n_{b}^{(1)}-\delta_{1} n_{b}^{(1)}+\sigma_{1} n_{d}^{(1)}-c_{1} n_{b}^{(1)} n_{b}^{(2)}+c_{1} \kappa_{1} n_{b}^{(2)} \\
& \frac{\partial n_{d}^{(2)}}{\partial t}=d_{2} \frac{\partial^{2} n_{d}^{(2)}}{\partial x^{2}}-e_{2} \frac{\partial n_{d}^{(2)}}{\partial x}+\delta_{2} n_{b}^{(2)}-\sigma_{2} n_{d}^{(2)} \\
& \frac{\partial n_{b}^{(2)}}{\partial t}=r_{2} n_{b}^{(2)}\left(t-\tau_{2}, x\right)-\frac{r_{2}\left(n_{b}^{(2)}\right)^{2}}{\kappa_{2}}-\delta_{2} n_{b}^{(2)}+\sigma_{2} n_{d}^{(2)}+c_{2} n_{b}^{(1)} n_{b}^{(2)}-c_{2} \kappa_{1} n_{b}^{(2)} .
\end{aligned}
$$

For this system, we denote

$$
\boldsymbol{\beta}=\left(\frac{\delta_{1} \kappa_{1}}{\sigma_{1}}, \kappa_{1}, \frac{\delta_{2} \kappa_{2}}{\sigma_{2}}, \kappa_{2}\right)
$$

and notice that $\mathbf{0}=(0,0,0,0)$ and $\boldsymbol{\beta}$ are equilibria of the cooperative system (4.2).

We denote the system (4.2) in a more general form of

$$
\frac{\partial \mathbf{u}}{\partial t}=\mathbf{D} \frac{\partial^{2} \mathbf{u}}{\partial x^{2}}-\mathbf{E} \frac{\partial \mathbf{u}}{\partial x}+\mathbf{f}(\mathbf{u}(\theta, \mathbf{t}, \mathbf{x}))
$$

where $\mathbf{D}=\operatorname{diag}\left\{d_{1}, 0, d_{2}, 0\right\}, \mathbf{E}=\operatorname{diag}\left\{e_{1}, 0, e_{2}, 0\right\}$,

$$
\mathbf{f}(\mathbf{u}(\theta, t, x))=\left(\begin{array}{c}
\delta_{1} u_{2}-\sigma_{1} u_{1} \\
r_{1} u_{2}\left(t-\tau_{1}, x\right)+\frac{r_{1} u_{2}{ }^{2}}{\kappa_{1}}-2 r_{1} u_{2}-\delta_{1} u_{2}+\sigma_{1} u_{1}-c_{1} u_{2} u_{4}+c_{1} \kappa_{1} u_{4} \\
\delta_{2} u_{4}-\sigma_{2} u_{3} \\
r_{2} u_{4}\left(t-\tau_{2}, x\right)-\frac{r_{2} u_{4}{ }^{2}}{\kappa_{2}}-\delta_{2} u_{4}+\sigma_{2} u_{3}+c_{2} u_{2} u_{4}-c_{2} \kappa_{1} u_{4},
\end{array}\right)
$$

and $\mathbf{u}:=\left(u_{1}, u_{2}, u_{3}, u_{4}\right)=\left(n_{d}^{(1)}, n_{b}^{(1)}, n_{d}^{(2)}, n_{b}^{(2)}\right)$. Here, $\theta$ appearing in the function $\mathbf{f}(\mathbf{u}(\theta, t, x))$ represents the presence of time delays involved in the growth and spread of the population. We use the notation

$$
\mathcal{C}_{\boldsymbol{\beta}}:=\{\mathbf{u}: \mathbf{u}(x) \text { is continuous, and } \mathbf{0} \leq \mathbf{u}(x) \leq \boldsymbol{\beta} \text { for all } x\}
$$

We are interested in a nonincreasing traveling wave solution $\mathbf{w}(c ; \theta, x-c t)=$ $\left(\phi_{d}\left(\theta_{1}, x-c t\right), \phi_{b}\left(\theta_{2}, x-c t\right), \psi_{d}\left(\theta_{3}, x-c t\right), \psi_{b}\left(\theta_{4}, x-c t\right)\right\}$ of our coorperative system (4.2) with speed $c$ connecting the equilibria $\mathbf{0}$ and $\boldsymbol{\beta}$. 
Let $\xi=x-c t$. The wave equation of the coorperative system (4.2) is

$$
\begin{aligned}
& d_{1} \phi_{d}^{\prime \prime}(\xi)+\left(c-e_{1}\right) \phi_{d}^{\prime}(\xi)+\delta_{1} \phi_{b}(\xi)-\sigma_{1} \phi_{d}(\xi)=0 \\
& c \phi_{b}^{\prime}(\xi)+r_{1} \phi_{b}\left(\xi-c \tau_{1}\right)+\frac{r_{1}\left(\phi_{b}(\xi)\right)^{2}}{\kappa_{1}}-2 r_{1} \phi_{b}(\xi)-\delta_{1} \phi_{b}(\xi)+\sigma_{1} \phi_{d}(\xi)-c_{1} \phi_{b}(\xi) \psi_{b}(\xi)+c_{1} \kappa_{1} \psi_{b}(\xi)=0 \\
& d_{2} \psi_{d}^{\prime \prime}(\xi)+\left(c-e_{2}\right) \psi_{d}^{\prime}(\xi)+\delta_{2} \psi_{b}(\xi)-\sigma_{2} \psi_{d}(\xi)=0 \\
& c \psi_{b}^{\prime}(\xi)+r_{2} \psi_{b}\left(\xi-c \tau_{2}\right)-\frac{r_{2}\left(\psi_{b}(\xi)\right)^{2}}{\kappa_{2}}-\delta_{2} \psi_{b}(\xi)+\sigma_{2} \psi_{d}(\xi)+c_{2} \phi_{b}(\xi) \psi_{b}(\xi)-c_{2} \kappa_{1} \psi_{b}(\xi)=0,
\end{aligned}
$$

with $\lim _{\xi \rightarrow-\infty} \mathbf{w}(\xi)=\mathbf{0}$ and $\lim _{\xi \rightarrow \infty} \mathbf{w}(\xi)=\boldsymbol{\beta}$.

Since some diffusion coefficients $d_{i}=0$ in the cooperative system (4.2), the compactness of solution operators cannot be guaranteed. Therefore we shall work on the integral equation corresponding to the cooperative system (4.2) in order to prove the existence of traveling wave solutions.

and $\mathbf{H}_{3}(\mathbf{u})=(\tilde{\mathbf{f}}(\mathbf{u})+\kappa \mathbf{u}) \frac{1}{\kappa}$ where

Choose $\kappa>0$. Define $\mathbf{H}_{1}(\mathbf{u})(\xi)=\frac{1}{\kappa}\left(\begin{array}{c}0 \\ r_{1} u_{2}(\xi) \\ 0 \\ 0\end{array}\right), \mathbf{H}_{2}(\mathbf{u})(\xi)=\frac{1}{\kappa}\left(\begin{array}{c}0 \\ 0 \\ 0 \\ r_{2} u_{4}(\xi)\end{array}\right)$,

$$
\tilde{\mathbf{f}}(\mathbf{u}(t, x))=\left(\begin{array}{c}
\delta_{1} u_{2}-\sigma_{1} u_{1} \\
\frac{r_{1} u_{2}^{2}}{\kappa_{1}}-2 r_{1} u_{2}-\delta_{1} u_{2}+\sigma_{1} u_{1}-c_{1} u_{2} u_{4}+c_{1} \kappa_{1} u_{4} \\
\delta_{2} u_{4}-\sigma_{2} u_{3} \\
-\frac{r_{2} u_{4}^{2}}{\kappa_{2}}-\delta_{2} u_{4}+\sigma_{2} u_{3}+c_{2} u_{2} u_{4}-c_{2} \kappa_{1} u_{4}
\end{array}\right) .
$$

There is a proper subset $\Sigma_{0}$ of $\{1, \ldots, k\}$ such that $d_{i}=0$ for $i \in \Sigma_{0}$ and $d_{i}>0$ for $i \notin \Sigma_{0}$. For $i \in \Sigma_{0}$, if $c>0$, define

$$
\left(\mathbf{m}_{c}\right)_{i}(x)= \begin{cases}0 & \text { when } x>0 \\ \frac{\kappa}{c} e^{\frac{\kappa}{c} x} & \text { when } x \leq 0,\end{cases}
$$

and if $c<0$, define

$$
\left(\mathbf{m}_{c}\right)_{i}(x)= \begin{cases}\frac{\kappa}{-c} e^{\frac{\kappa}{c} x} & \text { when } x \geq 0 \\ 0 & \text { when } x<0 .\end{cases}
$$


For $i \notin \Sigma_{0}$, define

$$
\left(\mathbf{m}_{c}\right)_{i}(x)=\frac{\kappa}{d_{i}\left(\lambda_{i 1}-\lambda_{i 2}\right)} \begin{cases}e^{-\lambda_{i 1} x} & \text { when } x \geq 0 \\ e^{-\lambda_{i 2} x} & \text { when } x<0\end{cases}
$$

where

$$
\begin{aligned}
& \lambda_{i 1}=\frac{\left(c-e_{i}\right)+\sqrt{\left(c-e_{i}\right)^{2}+4 \kappa d_{i}}}{2 d_{i}}>0, \\
& \lambda_{i 2}=\frac{\left(c-e_{i}\right)-\sqrt{\left(c-e_{i}\right)^{2}+4 \kappa d_{i}}}{2 d_{i}}<0 .
\end{aligned}
$$

Let

$$
\mathbf{m}_{c}(x)=\operatorname{diag}\left(\left(\mathbf{m}_{c}\right)_{1}(x), \ldots,\left(\mathbf{m}_{c}\right)_{k}(x)\right)
$$

We have that

$$
\int_{-\infty}^{\infty} \mathbf{m}_{c}(x) d x=\mathbf{I} .
$$

$\mathrm{Wu}$ and Zou [69] used $\left(\mathbf{m}_{\mathbf{c}}\right)_{i}$ defined above and studied traveling wave solutions for delayed reaction-diffusion systems with $d_{i}>0$ and $e_{i}=0$ for all $i$. Li [53] introduced the function similar to $\left(\mathbf{m}_{\mathbf{c}}\right)_{i}$ and investigated the existence of traveling wave solutions for the cooperative reacion-diffusion system without time delay.

It is easy to verify that each $\left(\mathbf{m}_{c}\right)_{i}(x)$ defined above has the properties that $\left(\mathbf{m}_{c}\right)_{i}(x) \geq 0,\left(\mathbf{m}_{c}\right)_{i}(x)$ is bounded, and $\int_{-\infty}^{+\infty}\left(\mathbf{m}_{c}\right)_{i}(x) d x=1$, so that $\left(\mathbf{m}_{c}\right)_{i}(x)$ represents a probability density function.

Define the integral system

$$
T_{c}[\mathbf{u}](x)=\int_{-\infty}^{\infty}\left[\mathbf{m}_{c}\left(x+c \tau_{1}-y\right) \mathbf{H}_{1}(\mathbf{u})(y)+\mathbf{m}_{c}\left(x+c \tau_{2}-y\right) \mathbf{H}_{2}(\mathbf{u})(y)+\mathbf{m}_{c}(x-y) \mathbf{H}_{3}(\mathbf{u})(y)\right] d y
$$

We shall consider the integral system (4.7) in the proof of traveling wave solution.

THEOREM 4.1. Assume that $d_{i} \geq 0$ for all $i$. Let $c \neq 0$ for all $i$ with $d_{i}=0$. Then $\mathbf{w}(x-c t)$ is a nonincreasing traveling wave solution of (4.2) connecting two different constant equilibria $\mathbf{0}$ and $\boldsymbol{\beta}$ if and only if $\mathbf{w}$ is a continuous nonincreasing function satisfying

$$
\mathbf{w}=T_{c}[\mathbf{w}](x)
$$


and connecting $\mathbf{0}$ and $\boldsymbol{\beta}$.

The proof of Theorem 4.1 is similar to the proof of Theorem 3.1 in [53], we thus omit the proof here. This theorem demonstrates that a traveling wave solution of the system (4.2) is equivalent to a fixed point of an operator of the compact integral system (4.7).

\subsection{Traveling Wave Solutions}

In this section we prove the existence of traveling wave solutions for the cooperative system (4.2). The next theorem gives us the important connection between traveling wave solutions of the cooperative system (4.2) and those of the integral system (4.7).

Define

$$
\mathbf{D}^{(l)}=\mathbf{D}+\left(\frac{1}{l}\right) \mathbf{I}
$$

with $l \geq 1$ and $\mathbf{I}$ the $4 \times 4$ identity matrix. Clearly, $\mathbf{D}^{(l)}$ approaches $\mathbf{D}$ as $l \rightarrow \infty$. And the solution map operators for

$$
\frac{\partial \mathbf{u}}{\partial t}=\mathbf{D}^{(l)} \frac{\partial^{2} \mathbf{u}}{\partial x^{2}}-\mathbf{E} \frac{\partial \mathbf{u}}{\partial x}+\mathbf{f}(\mathbf{u}(\mathbf{t}-\tau, \mathbf{x}))
$$

are compact.

\subsubsection{Recursions With Delay}

We use $\mathcal{H}$ to denote the habitat where the species grow, interact and migrate. $\mathcal{H}$ is either the real line (the continuous habitat) or the subset of the real line which consist of all integral multiples of positive mesh size $h$ (a discrete habitat). Let $\tau$ be a nonnegative real number. We use boldface Roman symbols such as $\mathbf{u}(\theta, x)$ to denote $k$-vector-valued functions of the two variables $\theta$ and $x$, and 
boldface Greek letters to stand for $k$-vectors, which may be considered as constant vector-valued functions. Here, $\mathbf{u} \geq \mathbf{v}$ means that $u^{i}(\theta, x) \geq v^{i}(\theta, x)$ for all $i=1,2, \ldots, k, \theta \in[-\tau, 0]$ and $x \in \mathcal{H}$, and $\mathbf{u} \gg \mathbf{v}$ means that $u^{i}(\theta, x)>v^{i}(\theta, x)$ for all $i, \theta$ and $x$. We also define $\max \{\mathbf{u}(\theta, x), \mathbf{v}(\theta, x)\}$ as the vector-valued function whose $i$ th component at $(\theta, x)$ is $\max \left\{u^{i}(\theta, x), v^{i}(\theta, x)\right\}$.

Let $\mathcal{C}$ be the set of all bounded continuous functions from $[-\tau, 0] \times \mathcal{H}$ to $\mathbb{R}^{k}, \overline{\mathcal{C}}$ be the set of all bounded continuous functions from $[-\tau, 0]$ to $\mathbb{R}^{k}$, and $\mathcal{X}$ be the set of bounded continuous functions from $\mathcal{H}$ to $\mathbb{R}^{k}$. If $\mathbf{r} \in \overline{\mathcal{C}}$ with $\mathbf{r} \gg \mathbf{0}$, we define the set of continuous functions

$$
\mathcal{C}_{\mathbf{r}}:=\{\mathbf{u} \in \mathcal{C}: \mathbf{0} \leq \mathbf{u} \leq \mathbf{r}\}
$$

Moreover, we define the metric function $d(\cdot, \cdot)$ in $\mathcal{C}$ by

$$
d(\phi, \psi)=\sum_{k=0}^{\infty} \frac{\max _{|x| \leq k, \theta \in[-\tau, 0]}|\phi(\theta, \mathbf{x})-\psi(\theta, \mathbf{x})|}{2^{k}} \forall \phi, \psi \in \mathcal{C}
$$

so that $(\mathcal{C}, d)$ is a metric space. The convergence of a sequence $\phi_{n}$ to $\phi$ with respect to this topology is equivalent to the uniform convergence of $\phi_{\mathbf{n}}$ to $\phi$ on bounded subsets of $[-\tau, 0] \times \mathcal{H}$.

We study the following discrete-time recursion with delay:

$$
\mathbf{u}_{n+1}=Q\left[\mathbf{u}_{n}\right], n=0,1,2, \ldots
$$

where $\mathbf{u}_{n}(\theta, x)=\left(u_{n}^{1}(\theta, x), u_{n}^{2}(\theta, x), \ldots, u_{n}^{k}(\theta, x)\right), \theta \in[-\tau, 0]$, and $x \in \mathcal{H}$ represents the population densities of $k$ species at time $n$ and point $x$ with time delay $\tau$. The operator $Q$ is said to be order - preserving if $\mathbf{u} \geq \mathbf{v}$ implies that $Q[\mathbf{u}] \geq Q[\mathbf{v}]$. A recursion (4.10) in which $Q$ has this property is said to be cooperative. A function is said to be an equilibrium of $Q$ if $Q[\mathbf{w}]=\mathbf{w}$, so that if $\mathbf{u}_{l}=\mathbf{w}$ in the recursion (4.10), then $\mathbf{u}_{n}=\mathbf{w}$ for all $n \geq l$. We shall study the evolution of the solution $\mathbf{u}_{n}$ of the recursion (4.10) from a $\mathbf{u}_{0}$ near an unstable constant equilibrium 
$\theta$. By introducing the new variable $\hat{\mathbf{u}}=\mathbf{u}-\theta$ if necessary, we shall assume the unstable equilibrium $\theta$ from which the system moves away is the origin $\mathbf{0}$.

We define the translation operator

$$
T_{y}[\mathbf{v}](\theta, x)=\mathbf{v}(\theta, x-y)
$$

A set $D \in \mathcal{C}_{\mathbf{r}}$ is said to be $T$-invariant if $T_{y}[D]=D$ for any $y \in \mathcal{H}$.

We shall make the following hypotheses on $Q$.

\section{HYPOTHESES 4.1.}

i. $Q[\mathbf{0}]=\mathbf{0}$, and there is a vector $\boldsymbol{\beta}(\theta) \in \overline{\mathcal{C}}$ with $\boldsymbol{\beta}(\theta) \gg \mathbf{0}$ such that $Q[\boldsymbol{\beta}]=\boldsymbol{\beta}$, and if $\mathbf{u}_{0}$ is any vector in $\overline{\mathcal{C}}$ with $\boldsymbol{\beta}(\theta) \gg \mathbf{u}_{0} \gg \mathbf{0}$, then the vector-valued function $\mathbf{u}_{n}$ obtained from the recursion (4.10) converges to $\boldsymbol{\beta}(\theta)$ uniformly on $[-\tau, 0]$ as $n$ approaches infinity.

ii. $Q$ is order-preserving on nonnegative functions, so that if $\mathbf{u} \geq \mathbf{v} \geq \mathbf{0}$, then $Q[\mathbf{u}] \geq Q[\mathbf{v}] \geq \mathbf{0}$

iii. $Q$ is translation invariant so that $Q\left[T_{y}[\mathbf{v}]\right]=T_{y}[Q[\mathbf{v}]]$ for all $y$.

iv. $Q$ is continuous with respect to the topology determined by $d(\cdot, \cdot)$ given in (4.9).

v. One of the following two properties holds:

a. $Q\left[\mathcal{C}_{\boldsymbol{\beta}}\right]$ is precompact in $\mathcal{C}_{\boldsymbol{\beta}}$.

b. The set $Q\left[\mathcal{C}_{\boldsymbol{\beta}}\right](0, \cdot)$ is precompact in $\mathcal{X}$, and there is a positive number $\zeta \leq \tau$ such that $Q[\mathbf{u}](\theta, x)=\mathbf{u}(\theta+\zeta, x)$ for all $\theta \in[-\tau,-\zeta]$, and the operator

$$
S[\mathbf{u}](\theta, x)= \begin{cases}\mathbf{u}(0, x), & \theta \in[-\tau,-\zeta), \\ Q[\mathbf{u}](\theta, x), & \theta \in[-\zeta, 0]\end{cases}
$$


has the property that $S[D]$ is precompact in $\mathcal{C}_{\boldsymbol{\beta}}$ for any $T$-invariant set $D \in \mathcal{C}_{\boldsymbol{\beta}}$ with $D(0, \cdot)$ precompact in $\mathcal{X}$.

Hypotheses 4.1 represents a revised set of the hypotheses given in [67].

REMARK 4.1. Hypotheses 4.1 i-ii imply that $Q$ takes $\mathcal{C}_{\boldsymbol{\beta}}$ into itself, and that the equilibrium $\boldsymbol{\beta}$ attracts all initial functions in $\mathcal{C}_{\boldsymbol{\beta}}$ with uniformly positive components. In biological terms, $\boldsymbol{\beta}$ is a globally asymptotically stable coexistence equilibrium. There may also be other equilibria lying between $\boldsymbol{\beta}$ and the extinction equilibrium $\mathbf{0}$, in each of which at least one of the species is extinct. In this paper, we assume that the recursion (4.10) has a finite number of equilibria and that the equilibria of (4.10) are completely separate in the sense that for any two equilibria $\boldsymbol{\nu}_{1}(\theta), \boldsymbol{\nu}_{2}(\theta) \in \overline{\mathcal{C}}$ of $(4.10)$, if $\nu_{1}^{i}(\theta) \neq \nu_{2}^{i}(\theta)$ for some $\theta \in[-\tau, 0]$, then $\nu_{1}^{i}(\theta) \neq \nu_{2}^{i}(\theta)$ for all $\theta \in[-\tau, 0]$.

REMARK 4.2. Li and Zhang [62] proposed the very similar hypotheses for their delayed cooperative systems. The difference between our system and theirs is that we divide each species into two compartments which results in the formation of two reaction-diffusion equations and two ordinary differential equations in our model. But those hypotheses given in [62] for the solution operator $Q$ can be applied for our model.

Before proving the existence of traveling wave solution of the system (4.2), we give the following lemma which is applied in our discussion and can be found in $[52,67,68]$.

LEMMA 4.1. (Comparison Lemma). Let $R$ be an order preserving operator. If $\mathbf{u}_{n}$ and $\mathbf{v}_{n}$ satisfy the inequalities $\mathbf{u}_{n} \leq R\left[\mathbf{u}_{n}\right]$ and $\mathbf{v}_{n} \geq R\left[\mathbf{v}_{n}\right]$ for all $n$, and if $\mathbf{u}_{0} \leq \mathbf{v}_{0}$, then $\mathbf{u}_{n} \leq \mathbf{v}_{n}$ for all $n$ 


\subsubsection{Existence of Traveling Wave Solutions}

Next, we prove the existence of traveling wave solutions of system (4.2). Our methods and arguments are highly inspired by the earlier works in $[53,67]$. However, our extended model is nontrivial and needs some new ideas and techniques such as taking the limit of a sequence of functions that are fixed points of the integral system.

Recall the cooperative system (4.2),

$$
\begin{aligned}
& \frac{\partial n_{d}^{(1)}}{\partial t}=d_{1} \frac{\partial^{2} n_{d}^{(1)}}{\partial x^{2}}-e_{1} \frac{\partial n_{d}^{(1)}}{\partial x}+\delta_{1} n_{b}^{(1)}-\sigma_{1} n_{d}^{(1)} \\
& \frac{\partial n_{b}^{(1)}}{\partial t}=r_{1} n_{b}^{(1)}\left(t-\tau_{1}, x\right)+\frac{r_{1}\left(n_{b}^{(1)}\right)^{2}}{\kappa_{1}}-2 r_{1} n_{b}^{(1)}-\delta_{1} n_{b}^{(1)}+\sigma_{1} n_{d}^{(1)}-c_{1} n_{b}^{(1)} n_{b}^{(2)}+c_{1} \kappa_{1} n_{b}^{(2)} \\
& \frac{\partial n_{d}^{(2)}}{\partial t}=d_{2} \frac{\partial^{2} n_{d}^{(2)}}{\partial x^{2}}-e_{2} \frac{\partial n_{d}^{(2)}}{\partial x}+\delta_{2} n_{b}^{(2)}-\sigma_{2} n_{d}^{(2)} \\
& \frac{\partial n_{b}^{(2)}}{\partial t}=r_{2} n_{b}^{(2)}\left(t-\tau_{2}, x\right)-\frac{r_{2}\left(n_{b}^{(2)}\right)^{2}}{\kappa_{2}}-\delta_{2} n_{b}^{(2)}+\sigma_{2} n_{d}^{(2)}+c_{2} n_{b}^{(1)} n_{b}^{(2)}-c_{2} \kappa_{1} n_{b}^{(2)},
\end{aligned}
$$

where $\mathbf{0}=(0,0,0,0)$ and $\boldsymbol{\beta}=\left(\frac{\delta_{1} \kappa_{1}}{\sigma_{1}}, \kappa_{1}, \frac{\delta_{2} \kappa_{2}}{\sigma_{2}}, \kappa_{2}\right)$ are the only two equilibria in $\mathcal{C}_{\boldsymbol{\beta}}$ when $r_{2}>c_{2} \kappa_{1}$, and the compact system (4.8)

$$
\frac{\partial \mathbf{u}}{\partial t}=\mathbf{D}^{(l)} \frac{\partial^{2} \mathbf{u}}{\partial x^{2}}-\mathbf{E} \frac{\partial \mathbf{u}}{\partial x}+\mathbf{f}(\mathbf{u}(\mathbf{t}-\tau, \mathbf{x}))
$$

DEFINITION 1. A function $\left(u_{d}(t, x), u_{b}(t, x), v_{d}(t, x), v_{b}(t, x)\right):[-\tau, b) \times \mathbb{R} \rightarrow \mathbb{R}^{4}, b>$ 0 , with the properties that $\left(u_{d}, u_{b}, v_{d}, v_{b}\right)$ is $\boldsymbol{C}^{2}$ in $x \in \mathbb{R}$ and $\boldsymbol{C}^{1}$ in $t \in(0, b)$ is called a super-solution (sub-solution) of (4.8) on $[0, b)$ if for $t \in[0, b), x \in \mathbb{R}$

$$
\begin{aligned}
\frac{\partial u_{d}}{\partial t} & \geq(\leq) d_{u_{d}} \frac{\partial^{2} u_{d}}{\partial x^{2}}-e_{1} \frac{\partial u_{d}}{\partial x}+\delta_{1} u_{b}-\sigma_{1} u_{d} \\
\frac{\partial u_{b}}{\partial t} & \geq(\leq) d_{u_{b}} \frac{\partial^{2} u_{b}}{\partial x^{2}}+r_{1} u_{b}\left(t-\tau_{1}, x\right)+\frac{r_{1}\left(u_{b}\right)^{2}}{\kappa_{1}}-2 r_{1} u_{b}-\delta_{1} u_{b}+\sigma_{1} u_{d}-c_{1} u_{b} v_{b}+c_{1} \kappa_{1} v_{b} \\
\frac{\partial v_{d}}{\partial t} & \geq(\leq) d_{v_{d}} \frac{\partial^{2} v_{d}}{\partial x^{2}}-e_{2} \frac{\partial v_{d}}{\partial x}+\delta_{2} v_{b}-\sigma_{2} v_{d} \\
\frac{\partial v_{b}}{\partial t} & \geq(\leq) d_{v_{b}} \frac{\partial^{2} v_{b}}{\partial x^{2}}+r_{2} v_{b}\left(t-\tau_{2}, x\right)-\frac{r_{2}\left(v_{b}\right)^{2}}{\kappa_{2}}-\delta_{2} v_{b}+\sigma_{2} v_{d}+c_{2} u_{b} v_{b}-c_{2} \kappa_{1} v_{b} .
\end{aligned}
$$

LEMMA 4.2. For any $\left(\phi_{d}, \phi_{b}, \psi_{d}, \psi_{b}\right) \in \mathcal{C}_{\boldsymbol{\beta}}$, system (4.8) has a unique classical solution

$\left(u_{d}\left(t, x ; \phi_{d}, \phi_{b}, \psi_{d}, \psi_{b}\right), u_{b}\left(t, x ; \phi_{d}, \phi_{b}, \psi_{d}, \psi_{b}\right), v_{d}\left(t, x ; \phi_{d}, \phi_{b}, \psi_{d}, \psi_{b}\right), v_{b}\left(t, x ; \phi_{d}, \phi_{b}, \psi_{d}, \psi_{b}\right)\right)$ for $(t, x) \in[\tau, \infty) \times \mathbb{R}$, where $\left(u_{d}(0, x), u_{b}(0, x), v_{d}(0, x), v_{b}(0, x)\right)=\left(\phi_{d}, \phi_{b}, \psi_{d}, \psi_{b}\right)$. 
Furthermore, for any pair of super-solution $\left(\overline{u_{d}}(t, x), \overline{u_{b}}(t, x), \overline{v_{d}}(t, x), \overline{v_{b}}(t, x)\right)$ and sub-solution $\left(\underline{u_{d}}(t, x), \underline{u_{b}}(t, x), \underline{v_{d}}(t, x), \underline{v_{b}}(t, x)\right)$ of (4.8) with

$$
\begin{aligned}
\mathbf{0}=(0,0,0,0) & \leq\left(\underline{u_{d}}(t, x), \underline{u_{b}}(t, x), \underline{v_{d}}(t, x), \underline{v_{b}}(t, x)\right) \\
& \leq\left(\overline{u_{d}}(t, x), \overline{u_{b}}(t, x), \bar{v}_{d}(t, x), \overline{v_{b}}(t, x)\right) \\
& \leq\left(\frac{\delta_{1} \kappa_{1}}{\sigma_{1}}, \kappa_{1}, \frac{\delta_{2} \kappa_{2}}{\sigma_{2}}, \kappa_{2}\right)=\boldsymbol{\beta}
\end{aligned}
$$

for $t \in[-\tau, 0]$ and $x \in \mathbb{R}$, the above compound inequality holds for $t \geq 0$ and $x \in \mathbb{R}$.

Proof. Define $f_{i}: \mathcal{C} \times \mathcal{C} \rightarrow \mathcal{X}, i=1,2,3,4$, by

$$
\begin{aligned}
f_{1}\left(\phi_{d}, \phi_{b}, \psi_{d}, \psi_{b}\right)(x)= & \delta_{1} \phi_{b}(0, x)-\sigma_{1} \phi_{d}(0, x) \\
f_{2}\left(\phi_{d}, \phi_{b}, \psi_{d}, \psi_{b}\right)(x)= & r_{1} \phi_{b}\left(-\tau_{1}, x\right)+\frac{r_{1} \phi_{b}^{2}(0, x)}{\kappa_{1}}-2 r_{1} \phi_{b}(0, x)-\delta_{1} \phi_{b}(0, x) \\
& +\sigma_{1} \phi_{d}(0, x)-c_{1} \phi_{b}(0, x) \psi_{b}(0, x)+c_{1} \kappa_{1} \psi_{b}(0, x) \\
f_{3}\left(\phi_{d}, \phi_{b}, \psi_{d}, \psi_{b}\right)(x)= & \delta_{2} \psi_{b}(0, x)-\sigma_{2} \psi_{d}(0, x) \\
f_{4}\left(\phi_{d}, \phi_{b}, \psi_{d}, \psi_{b}\right)(x)= & r_{2} \psi_{b}\left(-\tau_{2}, x\right)-\frac{r_{2} \psi_{b}^{2}(0, x)}{\kappa_{2}}-\delta_{2} \psi_{b}(0, x)+\sigma_{2} \psi_{d}(0, x) \\
& +c_{2} \phi_{b}(0, x) \psi_{b}(0, x)-c_{2} \kappa_{1} \psi_{b}(0, x) .
\end{aligned}
$$

The system (4.8) can be rewritten as

$$
\begin{array}{rlr}
\frac{\partial u_{d}}{\partial t} & =d_{u_{d}} \frac{\partial^{2} u_{d}}{\partial x^{2}}-e_{1} \frac{\partial u_{d}}{\partial x}+f_{1}\left(u_{d t}, u_{b t}, v_{d t}, v_{b t}\right)(x) \\
\frac{\partial u_{b}}{\partial t} & =d_{u_{b}} \frac{\partial^{2} u_{b}}{\partial x^{2}}+f_{2}\left(u_{d t}, u_{b t}, v_{d t}, v_{b t}\right)(x) \\
\frac{\partial v_{d}}{\partial t} & =d_{v_{d}} \frac{\partial^{2} v_{d}}{\partial x^{2}}-e_{2} \frac{\partial v_{d}}{\partial x}+f_{3}\left(u_{d t}, u_{b t}, v_{d t}, v_{b t}\right)(x) \\
\frac{\partial v_{b}}{\partial t} & =d_{v_{b}} \frac{\partial^{2} v_{b}}{\partial x^{2}}+f_{4}\left(u_{d t}, u_{b t}, v_{d t}, v_{b t}\right)(x), \quad t>0, x \in \mathbb{R},
\end{array}
$$

where $u_{d t}, u_{b t}, v_{d t}, v_{b t} \in \mathcal{C}$ with $u_{d t}(\theta, x)=u_{d}(t+\theta, x), u_{b t}(\theta, x)=u_{b}(t+\theta, x)$, $v_{d t}(\theta, x)=v_{d}(t+\theta, x)$ and $v_{b t}(\theta, x)=v_{b}(t+\theta, x)$ for $\theta \in[-\tau, 0], x \in \mathbb{R}$.

Let $\left\{T_{u_{d}}(t)\right\}_{t \geq 0},\left\{S_{u_{b}}(t)\right\}_{t \geq 0},\left\{T_{v_{d}}(t)\right\}_{t \geq 0}$ and $\left\{S_{v_{b}}(t)\right\}_{t \geq 0}$ be the solution 
semigroup on $\mathcal{X}$ generated by the heat equations

$$
\begin{aligned}
& u_{d, t}=d_{u_{d}} \Delta u_{d}-e_{1} u_{d, x}, \\
& u_{b, t}=d_{u_{b}} \Delta u_{b}, \\
& v_{d, t}=d_{v_{d}} \Delta v_{d}-e_{2} v_{d, x}, \text { and } \\
& v_{b, t}=d_{v_{b}} \Delta v_{b} .
\end{aligned}
$$

Then we can write (4.12) as the following integral equations:

$$
\begin{aligned}
& u_{d}(t, x)=T_{u_{d}}(t) u_{d}(0, \cdot)(x)+\int_{0}^{t} T_{u_{d}}(t-s) f_{1}\left(u_{d t}, u_{b t}, v_{d t}, v_{b t}\right)(x) d s \\
& u_{b}(t, x)=S_{u_{b}}(t) u_{b}(0, \cdot)(x)+\int_{0}^{t} S_{u_{b}}(t-s) f_{2}\left(u_{d t}, u_{b t}, v_{d t}, v_{b t}\right)(x) d s \\
& v_{d}(t, x)=T_{v_{d}}(t) v_{d}(0, \cdot)(x)+\int_{0}^{t} T_{v_{d}}(t-s) f_{3}\left(u_{d t}, u_{b t}, v_{d t}, v_{b t}\right)(x) d s \\
& v_{b}(t, x)=S_{v_{b}}(t) v_{b}(0, \cdot)(x)+\int_{0}^{t} S_{v_{b}}(t-s) f_{4}\left(u_{d t}, u_{b t}, v_{d t}, v_{b t}\right)(x) d s, \quad t>0
\end{aligned}
$$

where

$$
\begin{aligned}
& T_{u_{d}}(t)=\frac{1}{\sqrt{4 \pi d_{u_{d}} t}} \int_{-\infty}^{\infty} e^{-\frac{\left[(y-x)+e_{1} t\right]^{2}}{4 d_{u_{d}} t}} d y, \\
& S_{u_{b}}(t)=\frac{1}{\sqrt{4 \pi d_{u_{b}} t}} \int_{-\infty}^{\infty} e^{-\frac{(y-x)^{2}}{4 d_{u_{b}} t}} d y \\
& T_{v_{d}}(t)=\frac{1}{\sqrt{4 \pi d_{v_{d}} t}} \int_{-\infty}^{\infty} e^{-\frac{\left[(y-x)+e_{2} t\right]^{2}}{4 d_{v_{d}} t}} d y \\
& S_{v_{b}}(t)=\frac{1}{\sqrt{4 \pi d_{v_{b}} t}} \int_{-\infty}^{\infty} e^{-\frac{(y-x)^{2}}{4 d_{v_{b}} t}} d y .
\end{aligned}
$$

Under the abstract setting in Martin and Smith, a mild solution of (4.12) is a solution to its associated integral equation (4.13). One can easily verify that $f_{i}, \quad i=1,2,3,4$ are Lipschitz continuous on any bounded subset of $\mathcal{C} \times \mathcal{C}$. Let $\mathcal{Z}=\boldsymbol{B} \boldsymbol{U} \boldsymbol{C}\left(\mathbb{R}, \mathbb{R}^{4}\right)$ be the Banach space of all bounded and uniformly continuous functions from $\mathbb{R}$ into $\mathbb{R}^{4}$ with the usual supremum norm. Let $\mathcal{Z}^{+}=\left\{\left(\phi_{d 1}, \phi_{d 2}\right)\right.$ : $\left.\left(\phi_{d 1}, \phi_{d 2}\right) \in \mathcal{Z}, \phi_{d i}(x) \geq 0, i=1,2\right\}$. We claim that $f_{i}, i=1,2,3,4$ are quasi- 
monotone on $\mathcal{C}$ in the sense that

$$
\begin{aligned}
& \lim _{h \rightarrow 0^{+}} \frac{1}{h} \operatorname{dist}\left(\phi_{d 2}(0)-\phi_{d 1}(0)+h\left[f_{1}\left(\phi_{d 2}, \phi_{b 2}, \psi_{d 2}, \psi_{b 2}\right)-f_{1}\left(\phi_{d 1}, \phi_{b 1}, \psi_{d 1}, \psi_{b 1}\right)\right] ; \mathcal{Z}^{+}\right)=0 \\
& \lim _{h \rightarrow 0^{+}} \frac{1}{h} \operatorname{dist}\left(\phi_{b 2}(0)-\phi_{b 1}(0)+h\left[f_{2}\left(\phi_{d 2}, \phi_{b 2}, \psi_{d 2}, \psi_{b 2}\right)-f_{2}\left(\phi_{d 1}, \phi_{b 1}, \psi_{d 1}, \psi_{b 1}\right)\right] ; \mathcal{Z}^{+}\right)=0 \\
& \lim _{h \rightarrow 0^{+}} \frac{1}{h} \operatorname{dist}\left(\psi_{d 2}(0)-\psi_{d 1}(0)+h\left[f_{3}\left(\phi_{d 2}, \phi_{b 2}, \psi_{d 2}, \psi_{b 2}\right)-f_{3}\left(\phi_{d 1}, \phi_{b 1}, \psi_{d 1}, \psi_{b 1}\right)\right] ; \mathcal{Z}^{+}\right)=0 \\
& \lim _{h \rightarrow 0^{+}} \frac{1}{h} \operatorname{dist}\left(\psi_{b 2}(0)-\psi_{b 1}(0)+h\left[f_{4}\left(\phi_{d 2}, \phi_{b 2}, \psi_{d 2}, \psi_{b 2}\right)-f_{4}\left(\phi_{d 1}, \phi_{b 1}, \psi_{d 1}, \psi_{b 1}\right)\right] ; \mathcal{Z}^{+}\right)=0,
\end{aligned}
$$

for all $\phi_{d j}, \phi_{b j}, \psi_{d j}, \psi_{b j} \in \mathcal{C}_{\boldsymbol{\beta}}$ for $j=1,2$ with $\left(\phi_{d 2}, \phi_{b 2}, \psi_{d 2}, \psi_{b 2}\right) \geq\left(\phi_{d 1}, \phi_{b 1}, \psi_{d 1}, \psi_{b 1}\right)$. From the definitions of $f_{i}, i=1,2,3,4$ in (7.1) we see that

$$
\begin{aligned}
f_{1}\left(\phi_{d 2}, \phi_{b 2}, \psi_{d 2}, \psi_{b 2}\right) & -f_{1}\left(\phi_{d 1}, \phi_{b 1}, \psi_{d 1}, \psi_{b 1}\right) \\
& =\delta_{1} \phi_{b 2}(0, x)-\sigma_{1} \phi_{d 2}(0, x)-\left(\delta_{1} \phi_{b 1}(0, x)-\sigma_{1} \phi_{d 1}(0, x)\right) \\
& =\delta_{1}\left(\phi_{b 2}(0, x)-\phi_{b 1}(0, x)\right)-\sigma_{1}\left(\phi_{d 2}(0, x)-\phi_{d 1}(0, x)\right)
\end{aligned}
$$

and for sufficiently small $h>0$,

$$
\begin{aligned}
\phi_{d 2}(0, x)-\phi_{d 1}(0, x)+ & h\left[f_{1}\left(\phi_{d 2}, \phi_{b 2}, \psi_{d 2}, \psi_{b 2}\right)-f_{1}\left(\phi_{d 1}, \phi_{b 1}, \psi_{d 1}, \psi_{b 1}\right)\right] \\
= & \left(1-h \sigma_{1}\right)\left(\phi_{d 2}(0, x)-\phi_{d 1}(0, x)\right) \\
& +h \delta_{1}\left(\phi_{b 2}(0, x)-\phi_{b 1}(0, x)\right) \\
\geq & 0
\end{aligned}
$$

similarly,

$$
\begin{aligned}
\psi_{d 2}(0, x)-\psi_{d 1}(0, x)+ & h\left[f_{3}\left(\phi_{d 2}, \phi_{b 2}, \psi_{d 2}, \psi_{b 2}\right)-f_{3}\left(\phi_{d 1}, \phi_{b 1}, \psi_{d 1}, \psi_{b 1}\right)\right] \\
= & \left(1-h \sigma_{2}\right)\left(\psi_{d 2}(0, x)-\psi_{d 1}(0, x)\right) \\
& +h \delta_{2}\left(\psi_{b 2}(0, x)-\psi_{b 1}(0, x)\right) \\
\geq & 0 .
\end{aligned}
$$


For $f_{2}$,

$$
\begin{aligned}
f_{2}\left(\phi_{d 2}, \phi_{b 2}, \psi_{d 2}, \psi_{b 2}\right)- & f_{2}\left(\phi_{d 1}, \phi_{b 1}, \psi_{d 1}, \psi_{b 1}\right) \\
= & r_{1} \phi_{b 2}\left(-\tau_{1}, x\right)+\frac{r_{1} \phi_{b 2}^{2}(0, x)}{\kappa_{1}}-2 r_{1} \phi_{b 2}(0, x)-\delta_{1} \phi_{b 2}(0, x) \\
& +\sigma_{1} \phi_{d 2}(0, x)-c_{1} \phi_{b 2}(0, x) \psi_{b 2}(0, x)+c_{1} \kappa_{1} \psi_{b 2}(0, x) \\
& -\left[r_{1} \phi_{b 1}\left(-\tau_{1}, x\right)+\frac{r_{1} \phi_{b 1}^{2}(0, x)}{\kappa_{1}}-2 r_{1} \phi_{b 1}(0, x)-\delta_{1} \phi_{b 1}(0, x)\right. \\
& \left.+\sigma_{1} \phi_{d 1}(0, x)-c_{1} \phi_{b 1}(0, x) \psi_{b 1}(0, x)+c_{1} \kappa_{1} \psi_{b 1}(0, x)\right] \\
= & r_{1}\left(\phi_{b 2}\left(-\tau_{1}, x\right)-\phi_{b 1}\left(-\tau_{1}, x\right)\right)+\sigma_{1}\left(\phi_{d 2}(0, x)-\phi_{d 1}(0, x)\right) \\
& +c_{1} \kappa_{1}\left(\psi_{b 2}(0, x)-\psi_{b 1}(0, x)\right)+\left(\phi_{b 2}(0, x)-\phi_{b 1}(0, x)\right) \\
& \times\left[\frac{r_{1}}{\kappa_{1}}\left(\phi_{b 2}(0, x)+\phi_{b 1}(0, x)\right)-2 r_{1}-\delta_{1}-c_{1}\left(\psi_{b 2}(0, x)\right.\right. \\
& \left.\left.+\psi_{b 1}(0, x)\right)\right]+c_{1}\left(\phi_{b 2}(0, x) \psi_{b 1}(0, x)-\phi_{b 1}(0, x) \psi_{b 2}(0, x)\right)
\end{aligned}
$$

and for sufficiently small $h>0$,

$$
\begin{aligned}
\phi_{b 2}(0, x)- & \phi_{b 1}(0, x)+h\left[f_{2}\left(\phi_{d 2}, \phi_{b 2}, \psi_{d 2}, \psi_{b 2}\right)-f_{2}\left(\phi_{d 1}, \phi_{b 1}, \psi_{d 1}, \psi_{b 1}\right)\right] \\
= & h r_{1}\left(\phi_{b 2}\left(-\tau_{1}, x\right)-\phi_{b 1}\left(-\tau_{1}, x\right)\right)+h \sigma_{1}\left(\phi_{d 2}(0, x)-\phi_{d 1}(0, x)\right) \\
& +\left(\phi_{b 2}(0, x)-\phi_{b 1}(0, x)\right)\left\{1-h\left[2 r_{1}+\delta_{1}+c_{1}\left(\psi_{b 2}(0, x)\right.\right.\right. \\
& \left.\left.\left.+\psi_{b 1}(0, x)\right)-\frac{r_{1}}{\kappa_{1}}\left(\phi_{b 2}(0, x)+\phi_{b 1}(0, x)\right)-c_{1} \psi_{b 1}\right]\right\} \\
& +h c_{1}\left(\kappa_{1}-\phi_{b 1}\right)\left(\psi_{b 2}(0, x)-\psi_{b 1}(0, x)\right) \\
\geq & 0
\end{aligned}
$$

since $\boldsymbol{\beta}$ is the only interior equilibrium of (4.8). And

$$
\begin{aligned}
f_{4}\left(\phi_{d 2}, \phi_{b 2}, \psi_{d 2}, \psi_{b 2}\right)- & f_{4}\left(\phi_{d 1}, \phi_{b 1}, \psi_{d 1}, \psi_{b 1}\right) \\
= & r_{2} \psi_{b 2}\left(-\tau_{2}, x\right)-\frac{r_{2} \psi_{b 2}^{2}(0, x)}{\kappa_{2}}-\delta_{2} \psi_{b 2}(0, x)+\sigma_{2} \psi_{d 2}(0, x) \\
& +c_{2} \phi_{b 2}(0, x) \psi_{b 2}(0, x)-c_{2} \kappa_{1} \psi_{b 2}(0, x) \\
& -\left(r_{2} \psi_{b 1}\left(-\tau_{2}, x\right)-\frac{r_{2} \psi_{b 1}^{2}(0, x)}{\kappa_{2}}-\delta_{2} \psi_{b 1}(0, x)+\sigma_{2} \psi_{d 1}(0, x)\right. \\
& \left.+c_{2} \phi_{b 1}(0, x) \psi_{b 1}(0, x)-c_{2} \kappa_{1} \psi_{b 1}(0, x)\right) \\
= & r_{2}\left(\psi_{b 2}\left(-\tau_{2}, x\right)-\psi_{b 1}\left(-\tau_{2}, x\right)\right)+\sigma_{2}\left(\psi_{d 2}(0, x)-\psi_{d 1}(0, x)\right) \\
& +\left(\psi_{b 2}(0, x)-\psi_{b 1}(0, x)\right)\left[c_{2} \phi_{b 2}(0, x)-\frac{r_{2}}{\kappa_{2}}\left(\psi_{b 2}(0, x)\right.\right. \\
& \left.\left.+\psi_{b 1}(0, x)\right)-\delta_{2}-c_{2} \kappa_{1}\right]+c_{2} \psi_{b 1}(0, x)\left(\phi_{b 2}(0, x)-\phi_{b 1}(0, x)\right)
\end{aligned}
$$


and for sufficiently small $h>0$,

$$
\begin{aligned}
\psi_{b 2}(0, x)- & \psi_{b 1}(0, x)+h\left[f_{4}\left(\phi_{d 2}, \phi_{b 2}, \psi_{d 2}, \psi_{b 2}\right)-f_{4}\left(\phi_{d 1}, \phi_{b 1}, \psi_{d 1}, \psi_{b 1}\right)\right] \\
= & h r_{2}\left(\psi_{b 2}\left(-\tau_{2}, x\right)-\psi_{b 1}\left(-\tau_{2}, x\right)\right)+h \sigma_{2}\left(\psi_{d 2}(0, x)-\psi_{d 1}(0, x)\right) \\
& +\left(\psi_{b 2}(0, x)-\psi_{b 1}(0, x)\right)\left\{1-h\left[\frac{r_{2}}{\kappa_{2}}\left(\psi_{b 2}(0, x)+\psi_{b 1}(0, x)\right)+\delta_{2}\right.\right. \\
& \left.\left.+c_{2} \kappa_{1}-c_{2} \phi_{b 2}(0, x)\right]\right\}+h c_{2} \psi_{b 1}(0, x)\left(\phi_{b 2}(0, x)-\phi_{b 1}(0, x)\right) \\
\geq & 0 .
\end{aligned}
$$

It follows that (4.14) holds. By Corollary 5 in [45], we can show the existence and uniqueness of

$\left(u_{d}\left(t, x ; \phi_{d}, \phi_{b}, \psi_{d}, \psi_{b}\right), u_{b}\left(t, x ; \phi_{d}, \phi_{b}, \psi_{d}, \psi_{b}\right), v_{d}\left(t, x ; \phi_{d}, \phi_{b}, \psi_{d}, \psi_{b}\right), v_{b}\left(t, x ; \phi_{d}, \phi_{b}, \psi_{d}, \psi_{b}\right)\right)$

with

$$
\begin{aligned}
& \left(S_{1}(t, s), S_{2}(t, s), S_{3}(t, s), S_{4}(t, s)\right)=\left(S_{1}(t-s), S_{2}(t-s), S_{3}(t-s), S_{4}(t-s)\right) \\
& \left(T_{1}(t, s), T_{2}(t, s), T_{3}(t, s), T_{4}(t, s)\right)=\left(T_{1}(t-s), T_{2}(t-s), T_{3}(t-s), T_{4}(t-s)\right)
\end{aligned}
$$

for $t \geq s \geq 0$

$$
\begin{aligned}
& \left(B_{1}\left(t, \phi_{d}, \phi_{b}, \psi_{d}, \psi_{b}\right), B_{2}\left(t, \phi_{d}, \phi_{b}, \psi_{d}, \psi_{b}\right), B_{3}\left(t, \phi_{d}, \phi_{b}, \psi_{d}, \psi_{b}\right), B_{4}\left(t, \phi_{d}, \phi_{b}, \psi_{d}, \psi_{b}\right)\right) \\
= & \left(f_{1}\left(\phi_{d}, \phi_{b}, \psi_{d}, \psi_{b}\right), f_{2}\left(\phi_{d}, \phi_{b}, \psi_{d}, \psi_{b}\right), f_{3}\left(\phi_{d}, \phi_{b}, \psi_{d}, \psi_{b}\right), f_{4}\left(\phi_{d}, \phi_{b}, \psi_{d}, \psi_{b}\right)\right),
\end{aligned}
$$

and $v^{+}=\boldsymbol{\beta}, v^{-}=\mathbf{0}$. Moreover, by the semigroup theory given in the proof of Theorem 1 in [45], it follows that

$\left(u_{d}\left(t, x ; \phi_{d}, \phi_{b}, \psi_{d}, \psi_{b}\right), u_{b}\left(t, x ; \phi_{d}, \phi_{b}, \psi_{d}, \psi_{b}\right), v_{d}\left(t, x ; \phi_{d}, \phi_{b}, \psi_{d}, \psi_{b}\right), v_{b}\left(t, x ; \phi_{d}, \phi_{b}, \psi_{d}, \psi_{b}\right)\right)$

is a classical solution for $t>\tau$.

Let

$$
\Psi(\theta, x)=\left(\overline{u_{d}}(t, x), \overline{u_{b}}(t, x), \overline{v_{d}}(t, x), \overline{v_{b}}(t, x)\right)
$$

and

$$
\Phi(\theta, x)=\left(\underline{u_{d}}(t, x), \underline{u_{b}}(t, x), \underline{v_{d}}(t, x), \underline{v_{b}}(t, x)\right),
$$


$\theta \in[-\tau, 0], x \in \mathbb{R}$. Then $0 \leq \Phi \leq \Psi \leq \boldsymbol{\beta}$ with $\Phi \leq \Psi$ in $\mathcal{C}_{\boldsymbol{\beta}}$. Again by Corollary 5 in [45], we have

$$
\begin{aligned}
\mathbf{0} & \leq\left(u_{d}(t, x ; \Phi), u_{b}(t, x ; \Phi), v_{d}(t, x ; \Phi), v_{b}(t, x ; \Phi)\right) \\
& \leq\left(u_{d}(t, x ; \Psi), u_{b}(t, x ; \Psi), v_{d}(t, x ; \Psi), v_{b}(t, x ; \Psi)\right) \leq \boldsymbol{\beta}
\end{aligned}
$$

for $t>0, x \in \mathbb{R}$.

Let $v^{+}=\boldsymbol{\beta}$ and

$$
\begin{gathered}
v^{-}=\left(\underline{u_{d}}(t, x), \underline{u_{b}}(t, x), \underline{v_{d}}(t, x), \underline{v_{b}}(t, x)\right), \\
v^{+}=\left(\bar{u}_{d}(t, x), \bar{u}_{b}(t, x), \bar{v}_{d}(t, x), \bar{v}_{b}(t, x)\right)
\end{gathered}
$$

and $v^{-}=\mathbf{0}$, respectively, we obtain

$$
\begin{aligned}
& \left(\underline{u_{d}}(t, x), \underline{u_{b}}(t, x), \underline{v_{d}}(t, x), \underline{v_{b}}(t, x)\right) \\
\leq & \left(u_{d}(t, x ; \Phi), u_{b}(t, x ; \Phi), v_{d}(t, x ; \Phi), v_{b}(t, x ; \Phi)\right) \\
\leq & \boldsymbol{\beta}
\end{aligned}
$$

for $t \geq 0$ and $x \in \mathbb{R}$, and

$$
\begin{aligned}
\mathbf{0} & \leq\left(u_{d}(t, x ; \Psi), u_{b}(t, x ; \Psi), v_{d}(t, x ; \Psi), v_{b}(t, x ; \Psi)\right) \\
& \leq\left(\bar{u}_{d}(t, x), \bar{u}_{b}(t, x), \bar{v}_{d}(t, x), \bar{v}_{b}(t, x)\right)
\end{aligned}
$$

for $t \geq 0$ and $x \in \mathbb{R}$.

It follows from (4.15)-(4.17) that

$$
\left(\overline{u_{d}}(t, x), \overline{u_{b}}(t, x), \overline{v_{d}}(t, x), \overline{v_{b}}(t, x)\right) \geq\left(\underline{u_{d}}(t, x), \underline{u_{b}}(t, x), \underline{v_{d}}(t, x), \underline{v_{b}}(t, x)\right)
$$

for all $t \geq 0, x \in \mathbb{R}$. This completes the proof.

Lemma (4.2) together with the global stability results of system (4.8) shows that the time $t$ solution map $\mathbf{Q}_{t}$ of (4.8) with $t>0$ exists, and it satisfies Hypotheses $4.1 \mathrm{i}$-ii since (4.8) is an autonomous system. 
LEMMA 4.3. For any $t>0, \mathbf{Q}_{t}$ satisfies Hypothesis 4.1 iv with $\boldsymbol{\beta}$ given by (4.3).

Proof. Let $\Phi_{1}, \Phi_{2} \in \mathcal{C}_{\boldsymbol{\beta}}$. For any $\varepsilon>0$ and $t_{0}>0$, we define

$$
\begin{aligned}
& H(t, x):=\left|u_{d}\left(t, x ; \Phi_{1}\right)-u_{d}\left(t, x ; \Phi_{2}\right)\right|+\left|u_{b}\left(t, x ; \Phi_{1}\right)-u_{b}\left(t, x ; \Phi_{2}\right)\right| \\
& +\left|v_{d}\left(t, x ; \Phi_{1}\right)-v_{d}\left(t, x ; \Phi_{2}\right)\right|+\left|v_{b}\left(t, x ; \Phi_{1}\right)-v_{b}\left(t, x ; \Phi_{2}\right)\right| \\
& K:=\sup _{t \in\left[0, t_{0}\right], x \in \mathbb{R}} H(t, x) \\
& \Omega_{r}(z):=[-\tau, 0] \times[z-r, z+r], \forall r>0, z \in \mathbb{R} ; \\
& |\Phi|_{\Omega_{\rho}(z)}:=\sup _{(\theta, x) \in \Omega_{r}(z)}|\Phi(\theta, x)| \\
& \epsilon_{0}:=\frac{\varepsilon}{2(5+\Delta) t_{0} e^{\Delta t_{0}}}
\end{aligned}
$$

where $\Delta:=5 r_{1}+3 r_{2}+2\left(\delta_{1}+\delta_{2}+\sigma_{1}+\sigma_{2}\right)+\left(c_{1}+c_{2}\right)\left(2 \kappa_{1}+\kappa_{2}\right)$. Without loss of generality, we assume $K \geq \sup _{\theta \in[-\tau, 0], x \in \mathbb{R}} H(\theta, x)$. Then, there exists $\left(t^{*}, x^{*}\right) \in$ $\left[0, t_{0}\right] \times \mathbb{R}$ such that $H(\theta, x) \leq H\left(t^{*}, x^{*}\right)+\varepsilon_{0}$ for $(t, \theta, x) \in\left[0, t_{0}\right] \times[-\tau, 0] \times \mathbb{R}$.

Define

$$
\begin{array}{ll}
T_{u_{d}}(t)=\frac{1}{\sqrt{4 \pi d_{u_{d}} t}} \int_{-\infty}^{\infty} e^{-\frac{\left(y-x+e_{1} t\right)^{2}}{4 d_{u_{d}} t}} d y, & T_{v_{d}}(t)=\frac{1}{\sqrt{4 \pi d_{v_{d}} t}} \int_{-\infty}^{\infty} e^{-\frac{\left(y-x+e_{2} t\right)^{2}}{4 d_{v_{d}} t}} d y \\
S_{u_{b}}(t)=\frac{1}{\sqrt{4 \pi d_{u_{b}} t}} \int_{-\infty}^{\infty} e^{-\frac{(y-x)^{2}}{4 d_{u_{b}} t}} d y, & S_{v_{b}}(t)=\frac{1}{\sqrt{4 \pi d_{v_{b}} t}} \int_{-\infty}^{\infty} e^{-\frac{(y-x)^{2}}{4 d_{v_{b}} t}} d y
\end{array}
$$

Then

$$
\begin{aligned}
& u_{d}(\xi, t)=T_{u_{d}}(t) u_{d}(0, \cdot)(x)+\int_{0}^{t} T_{u_{d}}(t-s) f_{1}\left(u_{d s}, u_{b s}, v_{d s}, v_{b s}\right)(x) d s \\
& u_{b}(\xi, t)=S_{u_{b}}(t) u_{b}(0, \cdot)(x)+\int_{0}^{t} S_{u_{b}}(t-s) f_{2}\left(u_{d s}, u_{b s}, v_{d s}, v_{b s}\right)(x) d s \\
& v_{d}(\xi, t)=T_{v_{d}}(t) v_{d}(0, \cdot)(x)+\int_{0}^{t} T_{v_{d}}(t-s) f_{3}\left(u_{d s}, u_{b s}, v_{d s}, v_{b s}\right)(x) d s \\
& v_{b}(\xi, t)=S_{v_{b}}(t) v_{b}(0, \cdot)(x)+\int_{0}^{t} S_{v_{b}}(t-s) f_{4}\left(u_{d s}, u_{b s}, v_{d s}, v_{b s}\right)(x) d s .
\end{aligned}
$$


We choose $\sigma=\frac{\varepsilon}{8 e^{\Delta t_{0}}}$ and $M=M\left(\varepsilon, t_{0}\right)>0$ such that for any $t \in\left[0, t_{0}\right]$

$$
\begin{aligned}
& \int_{|y|>M} \frac{1}{\sqrt{4 \pi d_{u_{d}} t}} e^{-\frac{\left(y-x+e_{1} t\right)^{2}}{4 d_{u_{d}} t}} d y \leq \frac{\varepsilon_{0}}{u_{d}}, \\
& \int_{|y|>M} \frac{1}{\sqrt{4 \pi d_{v_{d}} t}} e^{-\frac{\left(y-x+e_{2} t\right)^{2}}{4 d_{v_{d}} t}} d y \leq \frac{\varepsilon_{0}}{v_{d}{ }^{*}}, \\
& \int_{|y|>M} \frac{1}{\sqrt{4 \pi d_{u_{b}} t}} e^{-\frac{(y-x)^{2}}{4 d_{u_{b}} t}} d y \leq \frac{\varepsilon_{0}}{u_{b}{ }^{*}}, \\
& \int_{|y|>M} \frac{1}{\sqrt{4 \pi d_{v_{b}} t}} e^{-\frac{(y-x)^{2}}{4 d_{v_{b}} t}} d y \leq \frac{\varepsilon_{0}}{v_{b} *}, \\
& T_{u_{d}}(t) H_{s}\left(0, x^{*}\right) \leq\left|H_{s}\right|_{\Omega_{M}\left(x^{*}\right)}+\varepsilon_{0}, \\
& T_{v_{d}}(t) H_{s}\left(0, x^{*}\right) \leq\left|H_{s}\right|_{\Omega_{M}\left(x^{*}\right)}+\varepsilon_{0}, \\
& S_{u_{b}}(t) H_{s}\left(0, x^{*}\right) \leq\left|H_{s}\right|_{\Omega_{M}\left(x^{*}\right)}+\varepsilon_{0}, \\
& S_{v_{b}}(t) H_{s}\left(0, x^{*}\right) \leq\left|H_{s}\right|_{\Omega_{M}\left(x^{*}\right)}+\varepsilon_{0}, \\
& S_{u_{b}}(t) H_{s}\left(-\tau_{1}, x^{*}\right) \leq\left|H_{s}\right|_{\Omega_{M}\left(x^{*}\right)}+\varepsilon_{0}, \\
& S_{v_{b}}(t) H_{s}\left(-\tau_{1}, x^{*}\right) \leq\left|H_{s}\right|_{\Omega_{M}\left(x^{*}\right)}+\varepsilon_{0} .
\end{aligned}
$$


For $i=1,2$ if $\left|\Phi_{1}(\theta, x)-\Phi_{1}(\theta, x)\right|_{\Omega_{M}\left(x^{*}\right)}<\sigma$, then

$$
\begin{aligned}
& \left|u_{d}\left(t^{*}, x^{*} ; \Phi_{1}\right)-u_{d}\left(t^{*}, x^{*} ; \Phi_{2}\right)\right| \\
= & \mid T_{u_{d}}\left(t^{*}\right) u_{d}\left(0, x^{*} ; \Phi_{1}\right) \\
& +\int_{0}^{t^{*}} T_{u_{d}}\left(t^{*}-s\right) f_{1}\left(u_{d s}\left(\cdot, \cdot ; \Phi_{1}\right), u_{b s}\left(\cdot, \cdot ; \Phi_{1}\right), v_{d s}\left(\cdot, \cdot ; \Phi_{1}\right), v_{b s}\left(\cdot, \cdot ; \Phi_{1}\right)\right)\left(x^{*}\right) d s \\
& -T_{u_{d}}\left(t^{*}\right) u_{d}\left(0, x^{*} ; \Phi_{2}\right) \\
& +\int_{0}^{t^{*}} T_{u_{d}}\left(t^{*}-s\right) f_{1}\left(u_{d s}\left(\cdot, \cdot ; \Phi_{2}\right), u_{b s}\left(\cdot, \cdot ; \Phi_{2}\right), v_{d s}\left(\cdot, \cdot ; \Phi_{2}\right), v_{b s}\left(\cdot, \cdot ; \Phi_{2}\right)\right)\left(x^{*}\right) d s \mid \\
\leq & T_{u_{d}}\left(t^{*}\right)\left|u_{d}\left(0, x^{*} ; \Phi_{1}\right)-u_{d}\left(0, x^{*} ; \Phi_{2}\right)\right| \\
& +\int_{0}^{t^{*}} T_{u_{d}}\left(t^{*}-s\right) \mid f_{1}\left(u_{d s}\left(\cdot, \cdot ; \Phi_{1}\right), u_{b s}\left(\cdot, \cdot ; \Phi_{1}\right), v_{d s}\left(\cdot, \cdot ; \Phi_{1}\right), v_{b s}\left(\cdot, \cdot ; \Phi_{1}\right)\right) \\
& -f_{1}\left(u_{d s}\left(\cdot, \cdot ; \Phi_{2}\right), u_{b s}\left(\cdot, \cdot ; \Phi_{2}\right), v_{d s}\left(\cdot, \cdot ; \Phi_{2}\right), v_{b s}\left(\cdot, \cdot ; \Phi_{2}\right)\right) \mid\left(x^{*}\right) d s \\
\leq & T_{u_{d}}\left(t^{*}\right) H\left(0, x^{*}\right)+\delta_{1} \int_{0}^{t^{*}} T_{u_{d}}\left(t^{*}-s\right) H_{s}\left(0, x^{*}\right) d s+\sigma_{1} \int_{0}^{t^{*}} T_{u_{d}}\left(t^{*}-s\right) H_{s}\left(0, x^{*}\right) d s \\
\leq & \sigma+\varepsilon_{0}+\delta_{1} \int_{0}^{t_{0}}\left(\left|H_{s}\right| \Omega_{M}\left(x^{*}\right)+\varepsilon_{0}\right) d s+\sigma_{1} \int_{0}^{t_{0}}\left(\left|H_{s}\right| \Omega_{M}\left(x^{*}\right)+\varepsilon_{0}\right) d s \\
\leq & +\varepsilon_{0}\left(1+\delta_{1} t_{0}+\sigma_{1} t_{0}\right)+\left(\delta_{1}+\sigma_{1}\right) \int_{0}^{t_{0}}\left|H_{s}\right| \Omega_{M}\left(x^{*}\right) d s .
\end{aligned}
$$

By a similar argument, we have

$$
\begin{aligned}
& \left|v_{d}\left(t^{*}, x^{*} ; \Phi_{1}\right)-v_{d}\left(t^{*}, x^{*} ; \Phi_{2}\right)\right| \\
& \leq \sigma+\varepsilon_{0}\left(1+\delta_{2} t_{0}+\sigma_{2} t_{0}\right)+\left(\delta_{2}+\sigma_{2}\right) \int_{0}^{t_{0}}\left|H_{s}\right|_{\Omega_{M}\left(x^{*}\right)} d s
\end{aligned}
$$


and

$$
\begin{aligned}
& \left|u_{b}\left(t^{*}, x^{*} ; \Phi_{1}\right)-u_{b}\left(t^{*}, x^{*} ; \Phi_{2}\right)\right| \\
& =\mid S_{u_{b}}\left(t^{*}\right) u_{b}\left(0, x^{*} ; \Phi_{1}\right) \\
& +\int_{0}^{t^{*}} S_{u_{b}}\left(t^{*}-s\right) f_{2}\left(u_{d s}\left(\cdot, \cdot ; \Phi_{1}\right), u_{b s}\left(\cdot, \cdot ; \Phi_{1}\right), v_{d s}\left(\cdot, \cdot ; \Phi_{1}\right), v_{b s}\left(\cdot, \cdot ; \Phi_{1}\right)\right)\left(x^{*}\right) d s \\
& -S_{u_{b}}\left(t^{*}\right) u_{b}\left(0, x^{*} ; \Phi_{2}\right) \\
& +\int_{0}^{t^{*}} S_{u_{b}}\left(t^{*}-s\right) f_{2}\left(u_{d s}\left(\cdot, \cdot ; \Phi_{2}\right), u_{b s}\left(\cdot, \cdot ; \Phi_{2}\right), v_{d s}\left(\cdot, \cdot ; \Phi_{2}\right), v_{b s}\left(\cdot, \cdot ; \Phi_{2}\right)\right)\left(x^{*}\right) d s \\
& \leq S_{u_{b}}\left(t^{*}\right)\left|u_{b}\left(0, x^{*} ; \Phi_{1}\right)-u_{b}\left(0, x^{*} ; \Phi_{2}\right)\right| \\
& +\int_{0}^{t^{*}} S_{u_{b}}\left(t^{*}-s\right) \mid f_{2}\left(u_{d s}\left(\cdot, \cdot ; \Phi_{1}\right), u_{b s}\left(\cdot, \cdot ; \Phi_{1}\right), v_{d s}\left(\cdot, \cdot ; \Phi_{1}\right), v_{b s}\left(\cdot, \cdot ; \Phi_{1}\right)\right) \\
& -f_{2}\left(u_{d s}\left(\cdot, \cdot ; \Phi_{2}\right), u_{b s}\left(\cdot, \cdot ; \Phi_{2}\right), v_{d s}\left(\cdot, \cdot ; \Phi_{2}\right), v_{b s}\left(\cdot, \cdot ; \Phi_{2}\right)\right) \mid\left(x^{*}\right) d s \\
& \leq S_{u_{b}}\left(t^{*}\right) H\left(0, x^{*}\right)+r_{1} \int_{0}^{t^{*}} S_{u_{b}}\left(t^{*}-s\right) H_{s}(-\tau, x) d s \\
& +\left(4 r_{1}+\delta_{1}\right) \int_{0}^{t^{*}} S_{u_{b}}\left(t^{*}-s\right) H_{s}\left(0, x^{*}\right) d s \\
& +\left(\sigma_{1}+2 c_{1} \kappa_{1}+c_{1} \kappa_{2}\right) \int_{0}^{t^{*}} S_{u_{b}}\left(t^{*}-s\right) H_{s}(-\tau, x) d s \\
& \leq \sigma+\varepsilon_{0}+r_{1} \int_{0}^{t_{0}}\left(\left|H_{s}\right|_{\Omega_{M}\left(x^{*}\right)}+\varepsilon_{0}\right) d s \\
& +\left(4 r_{1}+\delta_{1}+\sigma_{1}+2 c_{1} \kappa_{1}+c_{1} \kappa_{2}\right) \int_{0}^{t_{0}}\left(\left|H_{s}\right|_{\Omega_{M}\left(x^{*}\right)}+\varepsilon_{0}\right) d s \\
& =\sigma+\varepsilon_{0}\left[1+r_{1} t_{0}+\left(4 r_{1}+\delta_{1}+\sigma_{1}+2 c_{1} \kappa_{1}+c_{1} \kappa_{2}\right) t_{0}\right] \\
& +\left(5 r_{1}+\delta_{1}+\sigma_{1}+2 c_{1} \kappa_{1}+c_{1} \kappa_{2}\right) \int_{0}^{t_{0}}\left|H_{s}\right|_{\Omega_{M}\left(x^{*}\right)} d s .
\end{aligned}
$$

Similarly, we have

$$
\begin{aligned}
& \left|v_{b}\left(t^{*}, x^{*} ; \Phi_{1}\right)-v_{b}\left(t^{*}, x^{*} ; \Phi_{2}\right)\right| \\
& \leq \sigma+\varepsilon_{0}\left[1+r_{2} t_{0}+\left(2 r_{2}+\delta_{2}+\sigma_{2}+2 c_{2} \kappa_{1}+c_{2} \kappa_{2}\right) t_{0}\right] \\
& +\left(3 r_{2}+\delta_{2}+\sigma_{2}+2 c_{2} \kappa_{1}+c_{2} \kappa_{2}\right) \int_{0}^{t_{0}}\left|H_{s}\right|_{\Omega_{M}\left(x^{*}\right)} d s .
\end{aligned}
$$


Thus, we have

$$
\begin{aligned}
\left|H_{t}\right|_{\Omega_{M}\left(x^{*}\right)} \leq & \varepsilon_{0}+H\left(t^{*}, x^{*}\right) \\
= & \varepsilon_{0}+\left|u_{d}\left(t^{*}, x^{*} ; \Phi_{1}\right)-u_{d}\left(t^{*}, x^{*} ; \Phi_{2}\right)\right|+\left|u_{b}\left(t^{*}, x^{*} ; \Phi_{1}\right)-u_{b}\left(t^{*}, x^{*} ; \Phi_{2}\right)\right| \\
& +\left|v_{d}\left(t^{*}, x^{*} ; \Phi_{1}\right)-v_{d}\left(t^{*}, x^{*} ; \Phi_{2}\right)\right|+\left|v_{b}\left(t^{*}, x^{*} ; \Phi_{1}\right)-v_{b}\left(t^{*}, x^{*} ; \Phi_{2}\right)\right| \\
\leq & 4 \sigma+\varepsilon_{0}(5+\Delta) t_{0}+\Delta \int_{0}^{t_{0}}\left|H_{s}\right|_{\Omega_{M}\left(x^{*}\right)} d s .
\end{aligned}
$$

It follows by Gronwall's inequality that

$$
\left|H_{t}\right|_{\Omega_{M}\left(x^{*}\right)} \leq\left(4 \sigma+\varepsilon_{0}(5+\Delta) t_{0}\right) e^{\Delta t_{0}}, \forall t \in\left[0, t_{0}\right] .
$$

We then obtain that for any small $\varepsilon>0$, and compact subset $\zeta \subset[-\tau, 0] \times \mathbb{R}$, there exist $\sigma>0$ and a compact set $\Omega_{M}\left(x^{*}\right)$ such that $\zeta \subset \Omega_{M}\left(x^{*}\right)$ and

$$
\left|H_{t}\right|_{\zeta} \leq\left|H_{t}\right|_{\Omega_{M}\left(x^{*}\right)}<\varepsilon \text { for } t \in\left[0, t_{0}\right] \text { and }\left|\Phi_{1}-\Phi_{2}\right|_{\Omega_{M}\left(x^{*}\right)}<\sigma \text {. }
$$

This shows that $Q_{t}$ is continuous in $\Phi$ with respect to the compact open topology uniformly for $t \in\left[0, t_{0}\right]$. Note that the metric space $\left(\mathcal{C}_{\boldsymbol{\beta}}, d\right)$ is complete. By the triangle inequality and the continuity of $Q_{t}$ in $t$ from Lemma 4.2, it follows that $Q_{t}(\phi)$ is continuous in $(t ; \Phi)$ with respect to the compact open topology. This competes the proof of Lemma 4.3.

LEMMA 4.4. For any $t>0, \mathbf{Q}_{t}$ satisfies Hypothesis $4.1 v$ with $\boldsymbol{\beta}$ given by (4.3).

We can modify the proof of Lemma 3.3 in [62] by using the specific definition of $T_{u_{d}}, S_{u_{b}}, T_{v_{d}}$ and $S_{v_{b}}$ in this paper to prove Lemma 4.4. Since the argument is almost the same, we omit the proof.

LEMMA 4.5. Assume that $\mathbf{w}^{(l)}(c ; \theta, x-c t)$ is a nonincreasing traveling wave solution of (4.8) with speed $c \neq 0$ for $i \in \Sigma_{0}$. Then the family $\mathbf{w}^{(l)}$ is an equicontinuous family of functions. 
Proof. Define

$$
\left(\mathbf{m}_{c}^{(l)}\right)_{i}(x)=\frac{\kappa}{d_{i}^{(l)}\left(\lambda_{i 1}^{(l)}-\lambda_{i 2}^{(l)}\right)} \begin{cases}e^{-\lambda_{i 1}^{(l)} x} & \text { when } x \geq 0 \\ e^{-\lambda_{i 2}^{(l)} x} & \text { when } x<0\end{cases}
$$

with

$$
\begin{aligned}
& \lambda_{i 1}^{(l)}=\frac{\left(c-e_{i}\right)+\sqrt{\left(c-e_{i}\right)^{2}+4 \kappa d_{i}^{(l)}}}{2 d_{i}^{(l)}}>0, \\
& \lambda_{i 2}^{(l)}=\frac{\left(c-e_{i}\right)-\sqrt{\left(c-e_{i}\right)^{2}+4 \kappa d_{i}^{(l)}}}{2 d_{i}^{(l)}}<0 .
\end{aligned}
$$

By Theorem 4.1, we have

$$
\mathbf{w}^{(l)}(x)=\int_{-\infty}^{\infty} \mathbf{m}_{c}^{(l)}(x-y)\left[\mathbf{H}_{1}\left(\mathbf{w}^{(l)}\right)\left(y+c \tau_{1}\right)+\mathbf{H}_{2}\left(\mathbf{w}^{(l)}\right)\left(y+c \tau_{2}\right)+\mathbf{H}_{3}\left(\mathbf{w}^{(l)}\right)(y)\right] d y .
$$

Then we can follow the proof of Lemma 4.1 given in [53], therefore we omit the details here.

Define $\mathbf{R}_{c}[\mathbf{u}](\theta, s)=\max \left\{k \Phi(\theta, s), \mathbf{T}_{-c}[\mathbf{Q}[\mathbf{u}]](\theta, s)\right\}$ for $\mathbf{u} \in \mathcal{C}_{\boldsymbol{\beta}}$

Define a sequence of vector-valued functions $\mathbf{a}_{n}^{(l)}(c, k ; \theta, s)$ of $(\theta, s) \in[-\tau, 0] \times$ $\mathcal{H}$ by the recursion

$$
\mathbf{a}_{n+1}^{(l)}(c, k ; \theta, s)=\mathbf{R}_{c, k}\left[\mathbf{a}_{n}^{(l)}(c, k ; \cdot)\right](\theta, s), \quad \mathbf{a}_{0}^{(l)}(c, k ; \theta, s)=k \Phi(\theta, s) .
$$

and denote $\mathbf{a}^{(l)}$ as the limit of $\mathbf{a}_{n}^{(l)}(c, k ; \theta, s)$ as $n \rightarrow \infty$. Note that $\mathbf{a}_{n}^{(l)} \leq \mathbf{a}_{n+1}^{(l)} \leq \boldsymbol{\beta}$ for all $n$, and $\mathbf{a}_{n}^{(l)}(c, k ; \theta, s)$ is nonincreasing in $c$ and $s$ and continuous in $(c, k ; \theta, s)$.

Define

$$
c(l)^{*}:=\sup \left\{c: \mathbf{a}^{(l)}(c, k ; \theta, \infty)=\boldsymbol{\beta}\right\},
$$

and

$$
c(l)_{+}^{*}:=\sup \left\{c: \mathbf{a}^{(l)}(c, k ; \theta, \infty) \neq \mathbf{0}\right\} .
$$

Let

$$
\tilde{c}^{*}=\liminf _{l \rightarrow \infty} c(l)^{*}
$$


and

$$
\tilde{c}_{+}^{*}=\liminf _{l \rightarrow \infty} c(l)_{+}^{*}
$$

THEOREM 4.2. Assume that Hypotheses 4.1 are satisfied. Then the following statements are true for the system (4.2):

i. If $c \geq \tilde{c}^{*}$ and $c \neq 0$ for $i \in \Sigma_{0}$, there is a nonincreasing traveling wave solution $\mathbf{w}(x-c t)$ with $\mathbf{w}(c ; \theta,-\infty)=\boldsymbol{\beta}$ and $\mathbf{w}(c ; \theta, \infty)$ an equilibrium other than $\boldsymbol{\beta}$.

ii. If there is a nonincreasing traveling wave $\mathbf{w}(c ; \theta, x-c t)$ with $\mathbf{w}(c ; \theta,-\infty)=\boldsymbol{\beta}$ and $\mathbf{w}(c ; \theta, \infty)$ an equilibrium other than $\boldsymbol{\beta}$, then $c \geq \tilde{c}^{*}$.

Proof. As shown in the proof of Theorem 4.2 of Liang and Zhao [67], $\left\{\mathbf{a}_{n}^{(l)}(c, k ; \theta, s)\right.$ : $n \geq 1, k \in(0,1]\}$ is a family of equicontinuous functions of $(\theta, s)$ in any bounded subset of $[-\tau, 0] \times \mathcal{H}$. Since $\mathbf{a}_{n}^{(l)}$ is nondecreasing in $n$, the whole sequence $\mathbf{a}_{n}^{(l)}(c, k ; \theta, s)$ converges to a function $\mathbf{a}^{(l)}(c, k ; \theta, s)$ uniformly for $\theta \in[-\tau, 0]$ and $s$ on bounded sets. In particular, $\mathbf{a}^{(l)}(c, k ; \theta, s)$ is a continuous function of $(\theta, s)$. By hypothesis $4.1 \mathrm{iv}$, we take limits of (4.19) and we have

$$
\mathbf{a}^{(l)}(c, k ; \theta, s)=\max \left\{k \Phi(\theta, s), \mathbf{Q}\left[\mathbf{a}^{(l)}(c, k ; \cdot)\right](\theta, s+c)\right\}
$$

Fix $\theta_{0} \in[-\tau, 0]$. For any integer $g$ we define

$$
\mathbf{K}_{k}(g):=\frac{1}{2}\left[\mathbf{a}^{(l)}\left(c, k ; \theta_{0}, g\right)+\mathbf{a}^{(l)}\left(c, k ; \theta_{0}, g+1\right)\right]
$$

Note that $\mathbf{K}_{k}(g)$ is nonincreasing in $g$. Since $\mathbf{K}_{k}(-\infty)=\boldsymbol{\beta}\left(\theta_{0}\right)$ and $\mathbf{K}_{k}(\infty)=\mathbf{0}$, there exists $g_{k}$ such that

$$
\frac{N-2}{N}\left|\boldsymbol{\beta}\left(\theta_{0}\right)\right| \leq \mathbf{K}_{k}\left(g_{k}\right) \leq \frac{N-1}{N}\left|\boldsymbol{\beta}\left(\theta_{0}\right)\right|
$$

for some large positive integer $N$.

Now consider $\mathbf{a}^{(l)}\left(c, k ; \theta, s+g_{k}\right)$. 
Claim: $\mathbf{a}^{(l)}(c, k ; \theta, s)$ forms a family of equicontinuous function of $(\theta, s)$ in any bounded subsets of $[-\tau, 0] \times \mathcal{H}$.

For any $\theta_{1}, \theta_{2} \in[-\tau, 0]$ and real numbers $s_{1}$ and $s_{2}$, we have that for any positive integer $n$,

$$
\begin{aligned}
\left|\mathbf{a}^{(l)}\left(c, k ; \theta_{1}, s_{1}\right)-\mathbf{a}^{(l)}\left(c, k ; \theta_{2}, s_{2}\right)\right| \leq & \left|\mathbf{a}^{(l)}\left(c, k ; \theta_{1}, s_{1}\right)-\mathbf{a}_{n}^{(l)}\left(c, k ; \theta_{1}, s_{1}\right)\right| \\
& +\left|\mathbf{a}^{(l)}\left(c, k ; \theta_{2}, s_{2}\right)-\mathbf{a}_{n}^{(l)}\left(c, k ; \theta_{2}, s_{2}\right)\right| \\
& +\left|\mathbf{a}_{n}^{(l)}\left(c, k ; \theta_{1}, s_{1}\right)-\mathbf{a}_{n}^{(l)}\left(c, k ; \theta_{2}, s_{2}\right)\right|
\end{aligned}
$$

Since $\mathbf{a}_{n}^{(l)}$ increases to $\mathbf{a}^{(l)}$ uniformly on bounded sets and $\left\{\mathbf{a}_{n}^{(l)}(c, k ; \theta, s): n \geq\right.$ $1, k \in(0,1]\}$ is a family of equicontinuous functions of $(\theta, s)$, one can show that the above claim is true.

Thus we can find a sequence $k_{i} \rightarrow 0$ such that $\mathbf{a}^{(l)}\left(c, k_{i} ; \theta, s+g_{k_{i}}\right)$ converges uniformly for $\theta \in[-\tau, 0]$ and $s$ on bounded sets to a function $\mathbf{w}^{(l)}(c ; \theta, s)$ that is nonincreasing in $s$.

Taking limits in (4.22) with $k=k_{i}$ and $s=s+g_{k_{i}}-c$ and using the translation invariance of $\mathbf{Q}$, we find that

$$
\mathbf{w}^{(l)}(c ; \theta, s-c)=\mathbf{Q}\left[\mathbf{w}^{(l)}(c ; \cdot)\right](\theta, s)
$$

So $\mathbf{u}_{n}(\theta, s)=\mathbf{w}^{(l)}(c ; \theta, s-n c)$ is a traveling wave solution of the recursion $\mathbf{u}_{n+1}=$ $\mathbf{Q}\left[\mathbf{u}_{n}\right], n=0,1,2, \ldots$

Let $s$ approach to $-\infty$ in $(4.25)$, then

$\mathbf{w}^{(l)}(c ; \cdot,-\infty)=\lim _{n \rightarrow \infty} \mathbf{w}^{(l)}(c ; \cdot, s-(n+1) c)=\lim _{n \rightarrow \infty} \mathbf{Q}^{n}\left[\mathbf{w}^{(l)}\right](\cdot, s)=\mathbf{Q}\left[\mathbf{w}^{(l)}(c ; \cdot,-\infty)\right]$

for $\forall s \in \mathcal{H}$, and subsequently we consider $s$ approach to $-\infty$, we have

$$
\mathbf{w}^{(l)}(c ; \cdot, \infty)=\lim _{s \rightarrow \infty} \mathbf{w}^{(l)}(c ; \cdot, s-c)=\lim _{s \rightarrow \infty} \mathbf{Q}\left[\mathbf{w}^{(l)}\right](\cdot, s)=\mathbf{Q}\left[\mathbf{w}^{(l)}(c ; \cdot, \infty)\right]
$$

for $\forall s \in \mathcal{H}$. It follows that $\mathbf{w}^{(l)}(c ; \cdot, \pm \infty)$ are equilibria of $\mathbf{Q}$. 
The definition (4.23) shows that as $k_{i} \rightarrow 0$ the sequence $\mathbf{K}_{k_{i}}\left(g_{k_{i}}\right)$ converges to $\frac{1}{2}\left[\mathbf{w}^{(l)}\left(c ; \theta_{0}, 0\right)+\mathbf{w}^{(l)}\left(c ; \theta_{0}, 1\right)\right]$. Since $\mathbf{w}^{(l)}(c ; \theta, s)$ is nonincreasing in $s$,

$$
\frac{N-2}{N}\left|\boldsymbol{\beta}\left(\theta_{0}\right)\right| \leq \lim _{k_{i} \rightarrow 0} \mathbf{K}_{k_{i}}\left(g_{k_{i}}\right) \leq \mathbf{w}^{(l)}\left(c ; \theta_{0},-\infty\right)
$$

and

$$
\mathbf{w}^{(l)}\left(c ; \theta_{0}, \infty\right) \leq \lim _{k_{i} \rightarrow 0} \mathbf{K}_{k_{i}}\left(g_{k_{i}}\right) \leq \frac{N-1}{N}\left|\boldsymbol{\beta}\left(\theta_{0}\right)\right| .
$$

Thus, we have $\mathbf{w}^{(l)}\left(c ; \theta_{0}, \infty\right) \neq \boldsymbol{\beta}$ and $\mathbf{w}^{(l)}\left(c ; \theta_{0},-\infty\right)=\boldsymbol{\beta}$ due to the choice of $N$. We have proved that (4.8) has a nonincreasing traveling wave solution $\mathbf{w}^{(l)}(c ; \theta, s-$ $n c)$ with $\mathbf{w}^{(l)}\left(c ; \theta_{0},-\infty\right)=\boldsymbol{\beta}$ and $\mathbf{w}^{(l)}\left(c ; \theta_{0}, \infty\right)$, an equilibrium other than $\boldsymbol{\beta}$.

Since $\boldsymbol{\beta}$ is the only interior equilibrium of $\mathcal{C}_{\boldsymbol{\beta}}$, we can choose $\eta>0$ small enough such that there is no constant equilibrium other than $\boldsymbol{\beta}$ in the set $\{\mathbf{w} \in$ $\left.\mathcal{C}_{\boldsymbol{\beta}}:|\boldsymbol{\beta}-\mathbf{w}| \leq \eta\right\}$. By the Intermediate Value Theorem, it shows that there exists a real number $s^{*}$ at which $\left|\boldsymbol{\beta}-\mathbf{w}^{(l)}\left(\theta_{0}, s^{*}\right)\right|=\eta$, since as $s$ increases from $-\infty$ to $\infty$, the continuous function $\left|\boldsymbol{\beta}-\mathbf{w}^{(l)}\left(\theta_{0}, s\right)\right|$ increases from 0 to a positive real number. We can assume $s^{*}=0$ by translating if necessary, and we have

$$
\left|\boldsymbol{\beta}-\mathbf{w}^{(l)}\left(\theta_{0}, 0\right)\right|=\eta
$$

By Lemma 4.5, $\mathbf{w}^{(l)}$ is an equicontinuous family of functions. Then using Ascoli's Theorem, we have that $\mathbf{w}^{(l)}(x)$ has a subsequence $\left\{\mathbf{w}^{\left(l_{j}\right)}(x)\right\}$ such that $\left\{\mathbf{w}^{\left(l_{j}\right)}(x)\right\}$ converges to $\mathbf{w}(x)$ uniformly on every bounded interval. Clearly,

$$
\left|\boldsymbol{\beta}-\mathbf{w}\left(\theta_{0}, 0\right)\right|=\eta
$$

One can show that

$$
\lim _{l \rightarrow \infty} \int_{-\infty}^{\infty}\left|\mathbf{m}_{c}^{(l)}(x)-\mathbf{m}_{c}(x)\right| d x=\mathbf{0},
$$

where $\left(\mathbf{m}_{c}^{(l)}\right)_{i}(x)$ is defined in the proof of Lemma 4.5. The proof can be found in Li [53]. 
Observe that $\mathbf{w}^{\left(l_{j}\right)}$ satisfies

$$
\begin{aligned}
\mathbf{w}^{\left(l_{j}\right)}(x)= & \int_{-\infty}^{\infty} \mathbf{m}_{c}^{\left(l_{j}\right)}(x-y)\left[\mathbf{H}_{1}\left(\mathbf{w}^{\left(l_{j}\right)}\right)\left(y+c \tau_{1}\right)\right. \\
& \left.+\mathbf{H}_{2}\left(\mathbf{w}^{\left(l_{j}\right)}\right)\left(y+c \tau_{2}\right)+\mathbf{H}_{3}\left(\mathbf{w}^{\left(l_{j}\right)}\right)(y)\right] d y \\
= & \int_{-\infty}^{\infty} \mathbf{m}_{c}(x-y)\left[\mathbf{H}_{1}\left(\mathbf{w}^{\left(l_{j}\right)}\right)\left(y+c \tau_{1}\right)\right. \\
& \left.+\mathbf{H}_{2}\left(\mathbf{w}^{\left(l_{j}\right)}\right)\left(y+c \tau_{2}\right)+\mathbf{H}_{3}\left(\mathbf{w}^{\left(l_{j}\right)}\right)(y)\right] d y \\
& +\int_{-\infty}^{\infty}\left[\mathbf{m}_{c}^{\left(l_{j}\right)}(x-y)-\mathbf{m}_{c}(x-y)\right]\left[\mathbf{H}_{1}\left(\mathbf{w}^{\left(l_{j}\right)}\right)\left(y+c \tau_{1}\right)\right. \\
& \left.+\mathbf{H}_{2}\left(\mathbf{w}^{\left(l_{j}\right)}\right)\left(y+c \tau_{2}\right)+\mathbf{H}_{3}\left(\mathbf{w}^{\left(l_{j}\right)}\right)(y)\right] d y
\end{aligned}
$$

Taking limits of (4.28), we obtain

$$
\mathbf{w}(x)=\int_{-\infty}^{\infty} \mathbf{m}_{c}(x-y)\left[\mathbf{H}_{1}(\mathbf{w})\left(y+c \tau_{1}\right)+\mathbf{H}_{2}(\mathbf{w})\left(y+c \tau_{2}\right)+\mathbf{H}_{3}(\mathbf{w})(y)\right] d y
$$

By Theorem 4.1, $\mathbf{w}(x)$ is a traveling wave solution of (4.2) with speed $\tilde{c}^{*}$. The condition (4.26) and the definition of $\eta$ indicate that $\mathbf{w}(-\infty)=\boldsymbol{\beta}$ and $\mathbf{w}(\infty)$ is a constant equilibrium of (4.2) other than $\boldsymbol{\beta}$. This completes the proof of the statement (i) of the theorem.

The proof of the statement (ii) is similar to the second part of the proof of Theorem 3.1 in Li and Weinberger [61], we omit it here. So the proof of the theorem is completed.

REMARK 4.3. Assume that Hypotheses 4.1 are satisfied. Then the following statements are true for the system (4.2):

i. if $c \geq \tilde{c}_{+}^{*}$ and $c \neq 0$ for $i \in \Sigma_{0}$, there is a nonincreasing traveling wave solution $\mathbf{w}(x-c t)$ with $\mathbf{w}(c ; \theta, \infty)=\mathbf{0}$ and $\mathbf{w}(c ; \theta,-\infty)$ an equilibrium other than $\mathbf{0}$; and

ii. if there is a nonincreasing traveling wave $\mathbf{w}(c ; \theta, x-c t)$ with $\mathbf{w}(c ; \theta, \infty)=\mathbf{0}$ and $\mathbf{w}(c ; \theta,-\infty)=\boldsymbol{\beta}$, then $c \geq \tilde{c}_{+}^{*}$. 
The proof is similar to that of Theorem 4.2 and can be found in [53].

In this chapter, we first constructed the integral system by defining $\mathbf{H}_{1}(\mathbf{u})$, $\mathbf{H}_{2}(\mathbf{u}), \mathbf{H}_{3}(\mathbf{u})$ and $\mathbf{m}_{c}(x)$. Then we showed that a traveling wave solution of the cooperative system (4.2) with a proper speed is a fixed point of a compact integral operator. Finally we took the limit of a sequence of functions that are fixed points of the related integral system, and Theorem 4.2 and Remark 4.3 showed that the existence of traveling wave solutions can be established with speeds above two extended real numbers, $\tilde{c}^{*}$ and $\tilde{c}_{+}^{*}$. 


\section{CHAPTER 5 CONCLUSION AND FUTURE DIRECTIONS}

\subsection{Conclusion}

In this dissertation we formulated a system of delayed reaction-diffusion equations which modeled growth, spread and competition of two species with stage structure. This model is an extension of the time-delayed population system with stage structure by Zhang et al. [64]. However, there is no compartmentalization for both species in their model. The advantage of our model is that we take all factors of compartmentalization, stage structure and interaction between two species into consideration in order to provide a resolution to the drift paradox in stream ecology. To the best of our knowledge, this is the first model involving all these factors. The underlying dynamics are very complicated, however we can still give a complete description of the global stability and traveling wave solutions.

In Chapter 3 we analyzed the non-spatial system (3.1) in which the effect of spatial advection and diffusion is not involved. We obtained the existence and local stability criteria of the equilibria of the non-spatial system (3.1). Then using the fluctuation lemma and constructing sequences approaching equilibrium points we showed that the global stability of the equilibria in our model can be completely determined. The mathematical methods used in our proofs are inspired by Al-

Omari and Gourley's work [63]. By the study of the non-spatial system (3.1), we conclude that (i) the two interactive species with stage structure can persist 
in a stream; (ii) one species out-competes the other one, and the species cannot coexist; (iii) the two species can coexist and approach a stable population density in long term under certain conditions (i.e. the two mono-culture equilibria $E_{1}$ and $E_{2}$ are both unstable).

The spatial system (4.1) is analyzed in Chapter 4. We defined the integral system and showed that a traveling wave solution of a delayed cooperative reactiondiffusion system with a proper speed is a fixed point of a compact integral operator. Further, we established the existence of traveling wave solutions with speeds above two extended real numbers by taking a limit of a sequence of functions that are fixed points of related integral systems. In biology, traveling waves are spatial transitions from an unstable state to a stable one. In other words, the existence of traveling wave solutions connecting two equilibria means that the unstable equilibrium is took over by the stable one in space as time increases.

\subsection{Discussion}

In Chapter 4 we proved the existence of traveling wave solutions with speeds above two extended real numbers $\tilde{c}^{*}$ and $\tilde{c}_{+}^{*}$. We are interested in how $\tilde{c}^{*}$ is related to $c^{*}$ and how $\tilde{c}_{+}^{*}$ is related to $c_{+}^{*}$. We want to find conditions, under which the linear determinacy conditions given in Weinberger et al. [52] are satisfied by (4.2), such that $\tilde{c}^{*}=\tilde{c}_{+}^{*}=c^{*}=c_{+}^{*}$ and they are all equal to the unique spreading speed of $(4.2)$.

Li in [53] showed that $\tilde{c}^{*}=\tilde{c}_{+}^{*}=c^{*}=c_{+}^{*}=\bar{c}$ for the general partially degenerate cooperative reaction-diffusion system

$$
\frac{\partial \mathbf{u}}{\partial t}=D \frac{\partial^{2} \mathbf{u}}{\partial x^{2}}-E \frac{\partial \mathbf{u}}{\partial x}+\mathbf{f}(\mathbf{u}(t, x))
$$

where

$$
\bar{c}:=\inf _{\mu>0}(1 / \mu) \gamma_{1}(\mu)
$$


and $\gamma_{1}(\mu)$ is the principal eigenvalue of the first irreducible diagonal block of the moment generating matrix $C_{\mu}$ of the linearized system of (5.1). He showed that $\bar{c}$ can be characterized as the slowest speed of a class of traveling wave solutions by verifying the linear determinacy conditions under appropriate assumptions.

However, in our system (4.4) the time delay is considered in the vector function $\mathbf{f}(\mathbf{u}(\theta, \mathbf{t}, \mathbf{x}))$. Consequently the principal eigenvalue $\gamma_{1}(\mu)$ of $C_{\mu}$ and the corresponding eigenvector $\boldsymbol{\xi}(\mu)$ cannot be expressed explicitly. Thus the ideas used in [53] cannot be applied in our system.

In order to verify the linear determinacy conditions, one might first write the eigenvector $\boldsymbol{\xi}(\mu)$ as a function of the principal eigenvalue $\gamma_{1}(\mu)$. Then using the fact that the wave speed equation $\phi(\mu)=(1 / \mu) \gamma_{1}(\mu)$ is a convex function and thus the infimum in Eq. (5.2) exists, one might provide an estimation of $\gamma_{1}(\mu)$ and therefore show that the linear determinacy conditions are satisfied under appropriate assumptions.

Liang and Zhao [67] developed the analytical theory on the spreading speeds for delayed cooperative systems, which can be applied on our cooperative model $(4.2)$.

\subsection{Future Directions}

This model can be extended in several different directions:

1. Consider species interaction in the streamflow. In stream ecology, many studies have been done to study how different organisms interact with each other and to understand the interconnections and impacts $[21,70,71]$. Different interactions include competition among individuals of the same species, competition and predation between different species. We may add terms for the populations in the drift to indicate their interaction, and these 
terms can even be nonlinear (density-dependent). By analyzing the new model, one may predict the dynamics of species in the flow such as how the numbers of each species are influenced by its prey, its competitors, its predators, and even diseases.

2. Add age structure for adult individuals. New age structure among adult members can be considered in the model, i.e. dividing each adult population into two groups regarding their productivity, young adults and old adults. Only young and healthy adult members have strong productivity, and the old and unhealthy ones do not contribute to reproduction. One can define $n_{i}, n_{m y}$ and $n_{m o}$ to represent the population density of immature members, young mature members and old members, respectively. Notice that these three variables interact with each other.

3. Density-dependent diffusion rate. If organisms are either attracted to each other or repelled from one another, then we may replace the simple diffusion term by a biased random motion model [72]. In reality, organisms tend to join together at low densities and to estrange one another at high densities. Such a density-dependent response can be modeled by a function $\psi(u)$ of population density [73], where $\psi(u)$ is negative at low densities and positive at high densities and $u$ is the population density.

4. Consider an Allee effect. Allee effect is the positive relationship between population density and individual fitness [77]. In other words, at low density the population does not grow optimally. Since many species experience an Allee effect, we may add an Allee effect in the growth function of species. Wang et al. [74] claimed that systems with Allee effect show destabilization compared to a LotkaVolterra-type competitive system. However, they only conducted phase plane analysis and numerical simulation, and the time delay 
was not considered.

5. Consider stochastic fluctuations in population growth. Population dynamics is also determined by the environmental stochasticity, which includes climate effects, effects of human interaction and effects of food resources. For example, in the summer a population may grow favorably because of an abundant food supply and the agreeable climate, while in the winter they may experience a large number of deaths and low reproduction rate due to drought or low temperature. Our present model assumes that the environment is temporally constant, and we may use a stochastic variable representing the growth function to model these random effects $[75,76]$. 


\section{REFERENCES}

[1] A. E. Hershey, J. Pastor, B. J. Peterson, and G. W. Kling, 1993. Stable isotopes resolve the drift paradox for Baetis mayflies in an arctic river. Ecology $74,2315-2325$.

[2] K. Müller, 1954. Investigations on the organic drift in North Swedish streams. Report of the Institute of Freshwater Research, Drottningholm, vol. 34, pp. 133-148.

[3] K. Müller, 1982. The colonization cycle of freshwater insects. Oecologia 53, 202-207.

[4] R. F. Waters, 1972. The drift of stream insects. Annu. Rev. Entomol. 17, $253-272$.

[5] J. Lancaster, A. G. Hildrew, 1993a. Characterising instream flow refugia. Can. J. Fish. Aquat. Sci. 50, 1663-1675.

[6] J. Lancaster, A. G. Hildrew, 1993b. Flow refugia and the microdistribution of lotic macroinvertebrates. JNABS 12, 285-393.

[7] J. H. Winterbottom, S. E. Orton, A. G. Hildrew, 1997a. Field experiments on the mobility of benthic invertebrates in a southern English stream. Freshwater Biol. 38, 37-47.

[8] J. H. Winterbottom, S. E. Orton, A. G. Hidrew, J. Lancaster, 1997b. Field experiments on flow refugia in streams. Freshwater Biol. 37, 569-580. 
[9] L. L. Rempel, J. S. Richardson, M. C. Healey, 1999. Flow refugia for benthic marcoinvertebrates during flooding of a large river. JNABS 18, 24-48.

[10] J. Lancaster, 2000. Geometric scaling of microhabitat patches and their efficacy as refugia during disturbance. J. Anim. Ecol. 63, 442-457.

[11] D. C. Speirs, W. S. C. Gurney, 2001. Population persistence in rivers and estuaries. Ecology 82, 1219-1237.

[12] S. Humphries, G. D. Ruxton, 2002. Is there really a drift paradox? J. Anim. Ecol. 71, 151-154.

[13] S. G. Fisher, 1983. Succession in streams. Pages 7-21 in J. R. Barnes and G. W. Minshall, editors. Stream ecology. Plenum Press, New York, New York, USA.

[14] D. D. Williams, N. E. Williams, 1993. The upstream/downstream movement paradoxof lotic invertebrates: quantitative evidence from a Welsh mountain stream. Freshwater Biol. 30, 199-218.

[15] G. A. Bird, H. B. N. Hynes, 1981. Movements of adult insects near streams in southern Ontario. Hydrobiologia 77, 65-69.

[16] M. J. Winterbourn, A. L. M. Crowe, 2001. Flight activity of insects along a mountain stream: is directional flight adaptive? Freshwater Biol. 46, 14791489.

[17] J. M. Elliot, 1971. Upstream movements of benthic invertebrates in a Lake District stream. J. Anim. Ecol. 40, 235-252.

[18] N. L. Poff, J. V. Ward, 1992. Heterogeneous currents and algal resources mediate in situ foraging activity of a mobile stream grazer. Oikos 65, 465-487. 
[19] S. Humphries, G. D. Ruxton, 2002. Is there really a drift paradox? J. Anim. Ecol. 71, 151-154.

[20] B. R. Anholt, 1995. Density dependence resolves the stream drift paradox. Ecology 76, 2235-2239.

[21] J. D. Allan, 1995. Stream Ecology: Structure and Function of Running Waters. Chapman \& Hall, London.

[22] E. R. Siler, J. B. Wallace, S. L. Eggert, 2001. Long-term effects of resource limitation on stream invertebrate drift. Can. J. Fish Aquat. Sci. 58, 1624-1637.

[23] E. Pachepsky, F. Lutscher, R. M. Nisbet, and M. A. Lewis, 2005. Persistence, spread and the drift paradox. Theoretical Population Biology 67: 61-73.

[24] W. G. Aiello and H. I. Freedman, 1990. A time-delay model of single species growth with stage structure. Math. Biosci. 101, 139-153.

[25] M. G. Bulmer, 1977. Periodical insects. Am. Nat. 111, 1099-1117.

[26] R. A. Desharnais and L. Liu, 1987. Stable demographic limit cycles in a laboratory populations of Tribolium castaneum. J. Animal Ecol. 56, 885-906.

[27] M. E. Fisher and B. S. Goh, 1984. Stability results for delayed-recruitment models in population dynamics. J. Math. Biol. 19, 147-156.

[28] F. S. Anderson, 1960. Competition in populations of one age group. Biometrica $16,19-27$.

[29] H. J. Barclay and P. Van den Driessche, 1980. A model for a species with two life history stages and added mortality. Ecol. Model. 11, 157-166. 
[30] W. S. C. Gurney, R. M. Nisbet, and J. H. Lawton, 1983. The systematic formulation of tractable single species population models incorporating age structure. J. Animal Ecol. 52, 479-495.

[31] W. S. C. Gurney and R. M. Nisbet, 1985. Fluctuating periodicity, generation separation, and the expression of larval competition. Theor. Pop. Biol. 28, $150-180$.

[32] Yu. S. Koslesov, 1983. Properties of solutions of a class of equations with lag which describe the dynamics of change in the population of a species with the age structure taken into account. Math. USSR Sb. 45, 91-100.

[33] H. D. Landahl and B. D. Hanson, 1975. A three stage population model with cannibalism. Bull. Math. Biol. 37, 11-17.

[34] S. N. Wood, S. P. Blythe, W. S. C. Gurney, and R. M. Nisbet, 1989. Instability in mortality estimation schemes related to stage-structure population models. IMA J. Math. Appl. Med. Biol. 6, 47-68.

[35] K. Tognetti, 1975. The two stage stochastic model. Math. Biosci. 25, 195-204.

[36] R. A. Fisher, 1937. The wave of advance of advantageous genes. Ann. Eugenics 7, 353-369.

[37] A. Kolmogorov, I. Petrovskii, and N. Piscounov, 1937. A study of the diffusion equation with increase in the amount of substance, and its application to a biological problem. In V. M. Tikhomirov, editor, Selected Works of A. N. Kolmogorov I, pages 248C270. Kluwer 1991, ISBN 90-277-2796-1. Translated by V. M. Volosov from Bull. Moscow Univ., Math. Mech. 1, 1-25. 
[38] R. A. Fisher. The genetical theory of natural selection. Oxford University Press, 1930. Oxford University Press, USA, New Ed edition, 2000, ISBN 9780-19-850440-5, variorum edition, 1999, ISBN 0-19-850440-3.

[39] P. Grindrod, 1991. The theory and applications of reaction-diffusion equations: Patterns and waves. Oxford Applied Mathematics and Computing Science Series. The Clarendon Press Oxford University Press, New York, second edition, 1996 ISBN 0-19-859676-6; ISBN 0-19-859692-8.

[40] M. J. Ablowitz and A. Zeppetella, 1979. Explicit solutions of Fisher's equation for a special wave speed. Bull. Math. Biol. 41, 835-840.

[41] D. L. De Angelis, 1975. Global asymptotic stability criteria for models of density-dependent population growth. Journal of Theoretical Biology, 50.1, $35-43$.

[42] H. I. Freedman and K. Gopalsamy, 1986. Global stability in time-delayed single-species dynamics. Bulletin of Mathematical Biology, 48.5, 485-492.

[43] Y. Kuang, 1993. Delay Differential Equations with Applications in Population Dynamics. Math. in Science and Engineering, Academic Press 191.

[44] R. H. Martin, and H. L. Smith, 1990. Abstract functional-differential equations and reaction-diffusion systems. Transactions of the American Mathematical Society, 321.1, 1-44.

[45] R. H. Martin and H. L. Smith, 1991. Reaction-diffusion systems with time delays: monotonicity, invariance, comparison and convergence. J. reine angew. Math, 413.1, 35.

[46] W. G. Aiello and H. I. Freedman, 1990. A time-delay model of single-species growth with stage structure. Mathematical Biosciences, 101.2, 139-153. 
[47] D. G. Aronson and H. F. Weinberger, 1977. The asymptotic speed of propagation of a simple epidemic. Nonlinear diffusion (NSF-CBMS Regional Conf. Nonlinear Diffusion Equations, Univ. Houston, Houston, Tex., 1976), 1-23. Research Notes in Mathematics, 14. Pitman, London.

[48] D. G. Aronson and H. F. Weinberger, 1975. Nonlinear diffusion in population genetics, combustion, and nerve pulse propagation. Partial differential equations and related topics (Program, Tulane Univ., New Orleans, La., 1974), 5-49. Lecture Notes in Mathematics, 446. Springer, Berlin.

[49] D. G. Aronson and H. F. Weinberger, 1978. Multidimensional nonlinear diffusion arising in population genetics. Adv. in Math. 30, no. 1, 33-76.

[50] N. Shigesada and K. Kawasaki, 1997. Biological invasions: theory and practice. Oxford University Press, New York.

[51] H. F. Weinberger, 1982. Long-time behavior of a class of biological models. SIAM J. Math. Anal. 13, no. 3, 353-396.

[52] H. F. Weinberger, M. A. Lewis and B. Li, 2002. Analysis of linear determinacy for spread in cooperative models. J. Math. Biol. 45, 183-218.

[53] B. Li, 2012. Traveling wave solutions in partially degenerate cooperative reaction-diffusion systems. Journal of Differential Equations 252.9, 4842-4861.

[54] J. D. Murray, 2002. Mathematical biology. I. An introduction. 3rd ed. Interdisciplinary Applied Mathematics, 17. Springer, New York.

[55] J. D. Murray, 2003. Mathematical biology. II. Spatial models and biomedical applications. 3rd ed. Interdisciplinary Applied Mathematics, 18. Springer, New York. 
[56] A. I. Volpert and V. A. Volpert, 1994. Traveling wave solutions of parabolic systems. Translations of Mathematical Monographs, 140. American Mathematical Society, Providence, R. I..

[57] K. W. Schaaf, 1987. Asymptotic behavior and traveling wave solutions for parabolic functional differential equations. Trans. Amer. Math. Soc. 302.2, 587-615.

[58] H. L. Smith and X.-Q. Zhao, 2000. Global asymptotic stability of traveling waves in delayed reaction-diffusion equations. SIAM J. Math. Anal. 31.3, 514534 .

[59] H. R. Thieme and X.-Q. Zhao, 2003. Asymptotic speeds of spread and traveling waves for integral equations and delayed reaction-diffusion models. J. Differential Equations 195.2, 430-470.

[60] M. A. Lewis, B. Li, and H. F. Weinberger, 2002. Spreading speed and linear determinacy for two-species competition models. Journal of mathematical biology, 45.3, 219-233.

[61] B. Li, H. F. Weinberger, and M. A. Lewis, 2005. Spreading speeds as slowest wave speeds for cooperative systems. Mathematical biosciences, 196.1, 82-98.

[62] B. Li and L. Zhang, 2011. Travelling wave solutions in delayed cooperative systems. Nonlinearity, 24.6, 1759.

[63] J. F. M. Al-Omari and S. A. S. Gourley, 2003. Stability and traveling fronts in Lotka-Volterra competition models with stage structure. SIAM Journal on Applied Mathematics, 63.6, 2063-2086. 
[64] L. Zhang, B. Li, and J. Shang, 2012. Stability and travelling waves for a timedelayed population system with stage structure. Nonlinear Analysis: Real World Applications, 13.3, 1429-1440.

[65] K. Gopalsamy, 1992. Stability and oscillations in delay differential equations of population dynamics. Kluwer Academic Pub.

[66] W. M. Hirsch, H. Hanisch, and J. P. Gabriel, 1985. Differential equation models of some parasitic infections: methods for the study of asymptotic behavior. Communications on Pure and Applied Mathematics, 38.6, 733-753.

[67] X. Liang and X.-Q. Zhao, 2007. Asymptotic speeds of spread and traveling waves for monotone semiflows with applications. Comm. Pure Appl. Math. 60, 1-40. Erratum: 2008. Comm. Pure Appl. Math. 61, 137-138.

[68] R. Lui, 1989. Biological growth and spread modeled by systems of recursions. II. Biological theory. Math. Biosci. 93, 297-312.

[69] J. Wu and X. Zou, 2001. Traveling wave fronts of reaction-diffusion systems with delay. Journal of Dynamics and Differential Equations, 13.3, 651-687.

[70] D. D. Hart, 1992. Community organization in streams: the importance of species interactions, physical factors, and chance. Oecologia, 91.2, 220-228.

[71] D. C. Culver, D. W. Fong, and R. W. Jernigan, 1991. Species interactions in cave stream communities: experimental results and microdistribution effects. American Midland Naturalist, 364-379.

[72] W. S. C. Gurney, and R. M. Nisbet, 1975. The regulation of inhomogeneous populations. Journal of Theoretical Biology, 52.2, 441-457.

[73] D. G. Aronson, 1980. Density-dependent interaction-diffusion systems. Dynamics and modelling of reactive systems, 161-176. 
[74] G. Wang, X. G. Liang, and F. Z. Wang, 1999. The competitive dynamics of populations subject to an Allee effect. Ecological Modelling, 124.2, 183-192.

[75] K. Tognetti, 1975. The two stage stochastic population model. Mathematical Biosciences, 25.3, 195-204.

[76] M. G. Neubert, M. Kot, M., and M. A. Lewis, 1995. Dispersal and pattern formation in a discrete-time predator-prey model. Theoretical Population Biology, 48.1, 7-43.

[77] P. A. Stephens, W. J. Sutherland, and R. P. Freckleton, 1999. What is the Allee effect?. Oikos, 185-190. 


\section{CURRICULUM VITAE}

\section{Chunwei Wang}

\section{EDUCATION}

University of Louisville, Louisville, KY

Ph.D., Applied and Industrial Mathematics,

Summer 2013

M.A., Applied and Industrial Mathematics,

August 2011

Beijing University of Technology, Beijing, China

B.S., Information and Computing Sciences,

July 2005

\section{Professional Experience}

Graduate Teaching Assistant, University of Louisville, Louisville, KY

Math 111: College Algebra, Recitation Instructor

Aug. 2008 - May 2013

Math 105: Introduction to Contemporary Mathematics

Math 10\%: Finite Mathematics

Math 109: Elementary Statistics

Math 180: Elements of Calculus

Math 111, 105, 107, 180, Lecture Instructor

Summer 2009 - Summer 2012

Research Experience

May 2009 - May 2013

University of Louisville, Louisville, KY

Private Tutor and University of Louisville Math Lab Tutor

Louisville, KY

Aug. 2008 - Apr. 2013

Test Engineer

Jul. 2005 - Jul. 2007

Beyondsoft Corporation, Beijing, China

\section{Talks \& Presentations}

Introduction to Delay Differential Equations.

May 2010

Math Biology Seminar, University of Louisville, Louisville, KY

Optimization of The Temporary Commercial Network

For The 2008 Beijing Olympics.

Jul. 2005

Applied Math Seminar, Beijing University of Technology, Beijing, China 


\section{Awards}

Ken F. and Sandra S. Hohman Fellowship,

Aug. 2008 - May 2009

University of Louisville, Louisville, KY

Second Place Scholarship,

Aug. 2001 - Aug. 2004

Beijing University of Technology, Beijing, China

\section{Professional Memberships}

American Mathematical Society

Since 2008

Tri-State Actuarial Club

Since 2012 